\title{
q-Rung Orthopair Fuzzy Geometric Aggregation Operators Based on Generalized and Group-Generalized Parameters with Application to Water Loss Management
}

\author{
Muhammad Riaz ${ }^{1}{ }^{\oplus}$, Ayesha Razzaq ${ }^{1}$, Humaira Kalsoom ${ }^{2}{ }^{\infty}$, Dragan Pamučar $^{3}{ }^{\circledR}$, \\ Hafiz Muhammad Athar Farid ${ }^{1}$ (D) and Yu-Ming Chu ${ }^{4,5 *}$ \\ 1 Department of Mathematics, University of the Punjab, Lahore 54590, Pakistan; \\ mriaz.math@pu.edu.pk (M.R.); ayesharazzaq061@gmail.com (A.R.); hmatharfarid@gmail.com (H.M.A.F.) \\ 2 School of Mathematical Sciences, Zhejiang University, Hangzhou 310027, China; humaira87@zju.edu.cn \\ 3 Department of Logistics, University of Defence, 11000 Belgrade, Serbia; dragan.pamucar@va.mod.gov.rs \\ 4 Department of Mathematics, Huzhou University, Huzhou 313000, China \\ 5 Hunan Provincial Key Laboratory of Mathematical Modeling and Analysis in Engineering, \\ Changsha University of Science and Technology, Changsha 410114, China \\ * Correspondence: chuyuming@zjhu.edu.cn
}

Received: 5 July 2020; Accepted: 23 July 2020; Published: 27 July 2020

check for updates

\begin{abstract}
The notions of fuzzy set (FS) and intuitionistic fuzzy set (IFS) make a major contribution to dealing with practical situations in an indeterminate and imprecise framework, but there are some limitations. Pythagorean fuzzy set (PFS) is an extended form of the IFS, in which degree of truthness and degree of falsity meet the condition $0 \leq \breve{\Theta}^{2}(x)+\mathfrak{K}^{2}(x) \leq 1$. Another extension of PFS is a $\mathfrak{q}$-rung orthopair fuzzy set ( $\mathfrak{\mathfrak { q }}$-ROFS), in which truthness degree and falsity degree meet the condition $0 \leq \breve{\Theta}^{\mathfrak{q}}(x)+\mathfrak{K}^{\mathfrak{q}}(x) \leq 1,(\mathfrak{q} \geq 1)$, so they can characterize the scope of imprecise information in more comprehensive way. q́-ROFS theory is superior to FS, IFS, and PFS theory with distinguished characteristics. This study develops a few aggregation operators (AOs) for the fusion of $\mathfrak{q}$-ROF information and introduces a new approach to decision-making based on the proposed operators. In the framework of this investigation, the idea of a generalized parameter is integrated into the $\mathfrak{q}$-ROFS theory and different generalized $\mathfrak{q}$-ROF geometric aggregation operators are presented. Subsequently, the AOs are extended to a "group-based generalized parameter", with the perception of different specialists/decision makers. We developed q́-ROF geometric aggregation operator under generalized parameter and $\mathfrak{q}$-ROF geometric aggregation operator under group-based generalized parameter. Increased water requirements, in parallel with water scarcity, force water utilities in developing countries to follow complex operating techniques for the distribution of the available amounts of water. Reducing water losses from water supply systems can help to bridge the gap between supply and demand. Finally, a decision-making approach based on the proposed operator is being built to solve the problems under the $\mathfrak{q}-\mathrm{ROF}$ environment. An illustrative example related to water loss management has been given to show the validity of the developed method. Comparison analysis between the proposed and the existing operators have been performed in term of counter-intuitive cases for showing the liability and dominance of proposed techniques to the existing one is also considered.
\end{abstract}

Keywords: q-Rung orthopair fuzzy sets; geometric aggregation operators based on generalized and group-generalized parameters; water loss management; decision making 


\section{Introduction}

For many years, the issue of vague and imperfect information has been at the forefront. Information aggregation is the key factor for the decision management in the areas of business, management, engineering, psychology, social sciences, medical sciences, and artificial intelligence. Various problems in different areas aligned with vague and imprecise information. Modeling obscurities and data accumulation are most important components for the decision management in many areas comprising artificial intelligence, medical diagnosis, image processing i.e., it is extremely difficult challenge for experts to acquire precise decision without dealing with indeterminate and ambiguous data. Due to the critical, complex, subjective, and poorly structured nature of the issues themselves, many of the scientists contributions are directed to the area of building objective models of decision support. The reason for this phenomenon should be sought in the fact that modeling this class of problems requires correct mapping not only of the assessed alternatives/variants or scenarios. In such a case, experts must also consider the consequences of analyzing the decision problem from different perspectives and points of view taking into account several conflicting criteria. Water services, particularly in developing countries, continue to operate with considerable inefficiencies in terms of water and revenue losses. With increasing demand for water and scarcity, utilities require effective strategies to make optimum use of the available water resources. There are various options for reducing water loss. Deciding on which option to choose between conflicting multiple criteria and different stakeholder interests is a challenging task. One of the main challenges facing water utilities worldwide is the high levels of water losses in the distribution networks. According to the World Bank [1] study, approximately 32 billion $\mathrm{m}^{3}$ of treated water is lost yearly as leakage from urban water distribution systems around the world, while 16 billion $\mathrm{m}^{3}$ is lost but not paid for. They also guesstimate that these losses cost water utilities as much as US 14 billion \$ per year, with one-third occurring in developing countries. In the light of global pressure (climate change, urbanization, demand, scarcity, etc.) water utilities, particularly in developing countries, need to operate more effectively to provide sustainable water services. Water loss management (WLM) has become an important decision issue in meeting utility ̌s strategic goals. Whereas strategic planning (SP) has proven to be a valuable tool for sustainable urban water management [2], water utilities in developing countries often lack the necessary capabilities to carryout SP [3].

Water losses from water distribution systems (WDSs) have a major effect on the economic viability of urban water supplies and are perhaps the most important measure of their inefficiency. Its control encourages the efficient use of water as a valuable natural resource by allowing less water to be collected from the environment [4]. There is a broad variety of choices for handling and reducing.

Water losses, including the use of advanced techniques such as online monitoring, multi- parameter sensors, pressure control, and asset management. The entire method is complex. Costly, it needs trained personnel, requires various levels of collaboration and includes different stakeholder interests. Multi-criteria decision making (MCDM) methods are suggested to reduce the difficulty of this multi-criteria task [5]. A number of researchers have recently addressed water resource management and planning issues by applying various MCDM strategies, such as PROMETHEE (preference ranking organization method for enrichment evaluations) [6,7], ELECTRE II (elimination et choix traduisant la realite) [8], fuzzy TOPSIS (technique for the order preference by similarity to ideal solution) [9], and fuzzy AHP (analytic hierarchy process) [10].

Addressing this problem, the idea of the generalized $\mathfrak{q}$-rung orthopair fuzzy set ( $\mathfrak{q}$-ROFS) is presented in this study.

To facilitate our debate, the paper is categorized as follows: in Section 2, we provided some literature review about uncertain data modeling. In Section 3, some basis concepts including fuzzy set (FS), intuitionistic fuzzy set (IFS), Pythagorean fuzzy set (PFS), and q́-ROFS are presented. Moreover, some operational laws of $\mathfrak{q}$-ROFSs and $\mathfrak{q}$-ROFNs, accuracy function, score function of $\mathfrak{q}$-ROFNs and aggregation operators are also provided. In Section 4 , we introduce generalized q́ -rung orthopair fuzzy set (GQROFS). In Section 5, some q́-ROF geometric aggregation operator based on a generalized 
parameter are presented. Section 6 consists of some q́-ROF geometric aggregation operators based on a group-generalized parameter. In Section 6, we established an MCDM approach and presented a numerical example of the proposed method for water loss management. In Section 6, we compared the proposed operators with existing ones.

\subsection{Literature Review}

Traditionally, the information about an alternative has been believed to be a crisp number or linguistic number. Nevertheless, information can not be aggregated in a simple form due to its uncertainty. MCDM is a critical framework for decision making science, the purpose of which is to identify the most exceptional goals among the most feasible ones. The person needs to assess the choices made by different types of assessment criteria, such as crisp numbers and intervals, in the actual decision-making process. However, in many cases, due to the presence of a number of data anomalies that may arise due to lack of knowledge or human error, it is difficult for a person to choose the correct choice. Consequently, in order to measure these inconsistencies and to analyze the mechanism, a large number of theories have been suggested. To cope up with such situations, fuzzy set, which is an extended form of classical set, innovated by Zadeh [11] entrained a insurgence in mathematics. FS is a substantial model to make a distinction and assembling of the various challenges with ambiguous boundary. A FS is a collection of object, explicated by a truthness function which allocates a degree of truthness, whose range lies between 0 and 1 to each element. IFS, innovated by Atanassov [12] as an extended form of FS. Yager [13-15] established PFS, which is an extended form of IFS [12]. Ali et al. [16] provided certain characteristics of soft sets (SSs), rough sets(RSs), and fuzzy soft sets(FSSs). Wang et al. [17] introduced spatial multi-criteria approach for flood risk management in the Dongting Lake Region. Wang et al. [18] introduced Single valued neutrosophic sets. Cubic IF aggregation operators are established by Kaur and Garg [19]. TOPSIS technique on the basis of connection number under interval-valued IFS environment, presented by Kumar and Garg [20]. The notion of Pythagorean fuzzy number presented by Peng and Yang [21] and examined certain results for PFSs. Different PF-information measures and their enrollments are innovated by Peng et al. [22].

The concept of linear Diophantine fuzzy set (LDFS) and its enrollments in MCDMs was innovated by Riaz and Hashmi [23]. LDFS with indicative attributes improves the existing approaches and the decision experts (DEs) can select the grading values without any restriction. Riaz and Tehrim [24] introduced cubic bipolar fuzzy set with application to multi-criteria group decision making using geometric aggregation operators. Riaz and Tehrim [25] used a robust extension of VIKOR method for bipolar fuzzy sets using connection numbers of SPA theory based metric spaces. Sharma H. K. et al. [26] introduced a rough set approach for forecasting models. Petrovic and Kankaras [27] introduced a hybridized multi-criteria decision making approach for the selection and evaluation of criteria for determination of air traffic control radar position. Yager [28] established an idea of q́-ROFS which is extended form of PFS, in which the degree of truthness $\breve{\Theta}_{A}(x)$ and degree of falsity $\mathfrak{K}_{A}(x)$ satisfy the condition $0 \leq \breve{\Theta}_{A}(x)^{\mathfrak{q}}+\mathfrak{K}_{A}(x)^{\mathfrak{q}} \leq 1,(\mathfrak{q} \geq 1)$ and degree of indefiniteness is given by $\pi_{A}(x)=$ $\left(\breve{\Theta}_{A}(x)^{\mathfrak{q}}+\mathfrak{K}_{A}(x)^{\mathfrak{q}}-\breve{\Theta}_{A}(x)^{\mathfrak{q}} \mathfrak{K}_{A}(x)^{\mathfrak{q}}\right)^{1 / \mathfrak{q}}$.

Multi-criteria decision making (MCDM) with various fuzzy sets have been studied by; Peng et al. [29], Ali [30], Chen et al. [31], Chi and Lui [32], Feng et al. [33-36], Garg [37], Garg and Arora [38-41], Jose and Kuriaskose [42], Joshi [43], Karaaslan [44], Liu and Wang [45], Liu et al. [46], and Peng and Dai [47].

Riaz et al. [48-51] introduced the concepts of q-rung orthopair fuzzy prioritized aggregation operators, q-rung orthopair fuzzy hybrid aggregation operators, q-rung orthopair fuzzy information aggregation using Einstein operations, q-rung orthopair fuzzy Einstein prioritized aggregation operators with application towards multi-criteria group decision making (MCGDM). Aggregation operators and MCDM methods have been studied by; Xu [52], Xu and Cai [53], Xu [54], Yager [55], Ye [56,57], Zhan et al. [58,59], Zhang and Zhan [60,61], Zhang et al. [62], and Harrison et al. [5].

In realistic situations, different kinds of conditions are not completely fulfilled, as in MCDM issues, a preference of experts throughout the decision-making process is done entirely by his opinions and may result in the wrong decisions. In addition, the decision maker's priority is a characteristic of his own 
understanding and should be verified by some other senior specialist/decision maker. There are number of circumstances when the original data should be verified by some other specialist/decision expert.

(1) A patient can explain the symptoms to a doctor in accordance with his/her observations and circumstances. The actual details may not be authentic, in denouncing the symptoms. Otherwise this distortion factorized by a doctor, this would be conducive to an incorrect diagnosis. Respect of this, it might be most cautious to seek advice of another doctor to temperate the intensity of a patient's symptoms through a generalized parameter, which signifying the reliability of the provided data.

(2) For the selection of a manager for a firm, an unfair decision can be done by the individual's judgment, it must be confirmed by some other observer/decision maker by a general attribute corresponding to the situation.

(3) In every MCDM method, it is necessary to demonstrate prior evaluation by another specialist/decision expert in terms of generalized parameter to minimize the indeterminacy in the provided data and produce an indeterminate comportment more precise.

In such situations, the chances of mistakes in decision of the expert's field cannot be excluded. Consequently in these circumstances, there is a requirement of a generalize parameter, signifying an specialist's degree of confidence in the reliability of presented data to make the method very close to realistic circumstances substantially.

\section{Preliminaries}

In the presented section, we concisely review certain fundamentals of different sets which have been very helpful in understanding the contributions in the paper.

Definition 1 ([11]). Let $\check{Y}$ be a set of elements of universe and $\breve{\Theta}_{F}: \check{Y} \rightarrow[0,1]$ is a truthness mapping. The fuzzy set (FS) $\mathcal{F}$ is defined as,

$$
\mathcal{F}=\left\{\left(\widetilde{\cup}, \breve{\Theta}_{\mathcal{F}}(\widetilde{U})\right): \widetilde{U} \in \check{Y}\right\}
$$

where, $\breve{\Theta}_{\mathcal{F}}(\widetilde{\cup})$ is a truthness degree of $\widetilde{\cup}$. The accumulation of all FSs defined on $\check{\mathrm{Y}}$ is represented as $\mathbf{F}(\check{\mathrm{Y}})$.

Definition 2 ([12]). An intuitionistic fuzzy set (IFS) $\mathcal{I}$ defined on the universe $\check{Y}$ is the set of ordered triplets ,

$$
\mathcal{I}=\left\{\left(\widetilde{\mathbb{U}}, \breve{\Theta}_{\mathcal{I}}(\widetilde{\mathbb{U}}), \mathfrak{K}_{\mathcal{I}}(\widetilde{\mathbb{U}})\right): \widetilde{\mathbb{U}} \in \check{\mathrm{Y}}\right\}
$$

with the condition that $0 \leq \breve{\Theta}_{\mathcal{I}}(\widetilde{\mathbb{U}})+\mathfrak{K}_{\mathcal{I}}(\widetilde{\mathbb{U}}) \leq 1$, where $\breve{\Theta}_{\mathcal{I}}(\widetilde{\mathbb{U}})$ is the truthness degree and $\mathfrak{K}_{\mathcal{I}}(\widetilde{\mathbb{U}})$ is a degree of falsity of an alternative $\widetilde{U}$ to $\mathcal{I}$.

Definition 3. Let $\check{Y}$ be a collection of universal elements. The Pythagorean fuzzy set (PFS) $\widetilde{\mathcal{P}}$ on $\check{Y}$ is defined as,

$$
\widetilde{\mathcal{P}}=\left\{\left(\widetilde{\cup}, \breve{\Theta}_{\widetilde{\mathcal{P}}}(\widetilde{\cup}), \mathfrak{K}_{\widetilde{\mathcal{P}}}(\widetilde{\cup})\right): \widetilde{\mathbb{U}} \in \check{Y}\right\}
$$

with the condition that $0 \leq \breve{\Theta}_{\widetilde{\mathcal{P}}}^{2}(\widetilde{\mathbb{U}})+\mathfrak{K}_{\widetilde{\mathcal{P}}}^{2}(\widetilde{\mathbb{U}}) \leq 1$ where $\breve{\Theta}_{\widetilde{\mathcal{P}}}(\widetilde{\mathbb{U}}): \check{Y} \rightarrow[0,1]$ is an indication of truthness degree and $\mathfrak{K}_{\widetilde{\mathcal{P}}}(\widetilde{\cup}): \check{Y} \rightarrow[0,1]$ indicates the degree of falsity of an universal element $\widetilde{\mathbb{U}} \in \check{Y}$. The degree of indeterminacy is given as $\pi_{\widetilde{\mathcal{P}}}(\widetilde{\cup})=\left(\breve{\Theta}_{\widetilde{\mathcal{P}}}^{2}(\widetilde{\cup})+\mathfrak{K}_{\widetilde{\mathcal{P}}}^{2}(\widetilde{\cup})-\breve{\Theta}_{\widetilde{\mathcal{P}}}^{2}(\widetilde{\mathbb{U}}) \mathfrak{K}_{\widetilde{\mathcal{P}}}^{2}(\widetilde{\cup})\right)^{1 / 2}$. For assistance, a fundamental component $\left\langle\breve{\Theta}_{\widetilde{\mathcal{P}}}, \mathfrak{K}_{\widetilde{\mathcal{P}}}\right\rangle$ in a PFS is called a PF-Number (PFN).

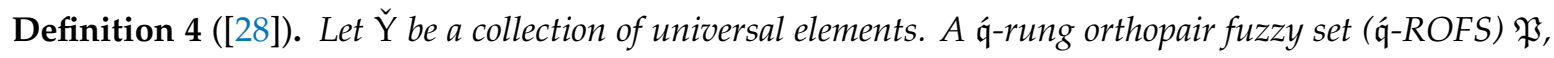
is characterized as

$$
\mathfrak{P}=\left\{\left(\widetilde{\mathbb{U}}, \breve{\Theta}_{\mathfrak{P}}(\widetilde{\mathbb{U}}), \mathfrak{K}_{\mathfrak{P}}(\widetilde{\mathbb{U}})\right): \widetilde{\mathbb{U}} \in \check{Y}\right\}
$$


with the condition that $0 \leq \breve{\Theta}_{\mathfrak{P}}^{\mathfrak{q}}(\widetilde{\mathbb{U}})+\mathfrak{K}_{\mathfrak{P}}^{\mathfrak{q}}(\widetilde{\cup}) \leq 1,(\dot{\mathfrak{q}} \geq 1)$, where $\breve{\Theta}_{\mathfrak{P}}(\widetilde{\mathbb{U}}): \check{Y} \rightarrow[0,1]$ indicates the truthness degree and $\mathfrak{K}_{\mathfrak{P}}(\widetilde{\mathbb{U}}): \check{Y} \rightarrow[0,1]$ indicates the degree of falsity of an alternative $\widetilde{U} \in \check{Y}$. The degree of indeterminacy is given as $\pi_{\mathfrak{P}}(\widetilde{\mathbb{U}})=\left(\breve{\Theta}_{\mathfrak{P}}^{\mathfrak{q}}(\widetilde{\cup})+\mathfrak{K}_{\mathfrak{P}}^{\mathfrak{q}}(\widetilde{\cup})-\breve{\Theta}_{\mathfrak{P}}^{\mathfrak{q}}(\widetilde{\mathbb{U}}) \mathfrak{K}_{\mathfrak{P}}^{\mathfrak{q}}(\widetilde{\cup})\right)^{1 / \mathfrak{q}}$.

For convenience, a basic element $\left\langle\breve{\Theta}_{\mathfrak{P}}(\widetilde{\cup}), \mathfrak{K}_{\mathfrak{P}}(\widetilde{\cup})\right\rangle$ in a $\mathfrak{\mathfrak { q }}-R O F$ is denoted by $\widetilde{\Xi}=\left\langle\breve{\Theta}_{\mathfrak{P}}, \mathfrak{K}_{\mathfrak{P}}\right\rangle$ for short, which is called ( $\mathfrak{q}-R O F N)$.

The proposed models of aggregated operators are credible, valid, versatile, and superior to others since they are based on the generalized q-ROFN structure. Whether the proposed operators are used in the sense of IFNs or PFNs, the results may be imprecise due to the lack of information in the input data. This loss is due to limitations on membership and non-membership of IFNs and PFNs (see Figure 1). IFNs and PFNs are special cases of $q$-ROFNs where $q=1$ and $q=2$, respectively.

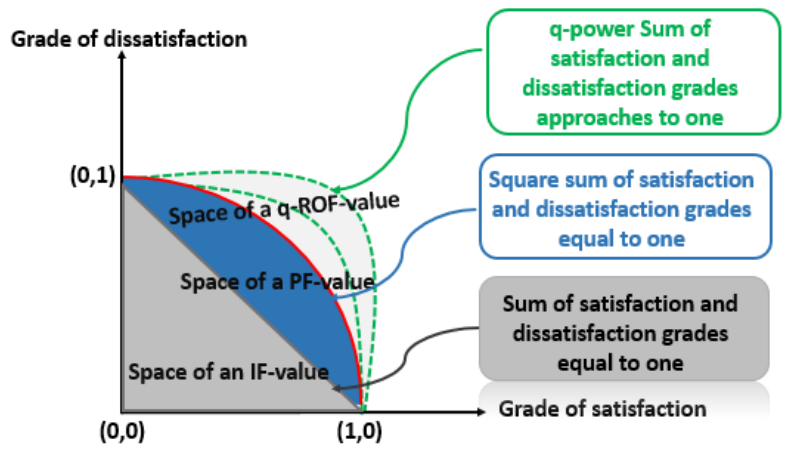

Figure 1. Graphical comparison between the IF-value, PF-value, and q-ROF-value.

\subsection{Operational Laws of $\hat{\mathfrak{q}}-R O F S$}

Let $\hat{\beth}_{1}=\left\langle\breve{\Theta}_{\beth_{1}}(\widetilde{U}), \mathfrak{K}_{\beth_{1}}(\widetilde{U})\right\rangle$ and $\hat{\beth}_{2}=\left\langle\breve{\Theta}_{\beth_{2}}(\widetilde{U}), \mathfrak{K}_{\beth_{2}}(\widetilde{U})\right\rangle$ be $\mathfrak{q}-R O F S$ s on $\check{Y}$. Then,

(1) $\quad \overline{\bar{\beth}_{1}}=\left\langle\mathfrak{K}_{\beth_{1}}(\widetilde{\cup}), \breve{\Theta}_{\beth_{1}}(\widetilde{\cup})\right\rangle$.

(2) $\quad \breve{\beth}_{1} \widetilde{\simeq} \breve{\beth}_{2} \Leftrightarrow \breve{\Theta}_{\mathfrak{\beth}_{1}}(\widetilde{\mathbb{U}}) \leqslant \breve{\Theta}_{\mathfrak{\beth}_{2}}(\widetilde{U})$ and $\mathfrak{K}_{\mathfrak{\beth}_{2}}(\widetilde{\mathbb{U}}) \leqslant \mathfrak{K}_{\mathfrak{\beth}_{1}}(\widetilde{\mathbb{U}})$.

(3) $\hat{\beth}_{1}=\hat{\beth}_{2} \Leftrightarrow \hat{\beth}_{1} \widetilde{\subseteq} \hat{\beth}_{2}$ and $\hat{\beth}_{2} \widetilde{\simeq} \hat{\beth}_{1}$.

(4) $\vec{\beth}_{1} \widetilde{\sqcup} \hat{\beth}_{2}=\left\{\left\langle\widetilde{U}, \max \left\{\breve{\Theta}_{\beth_{1}}(\widetilde{U}), \breve{\Theta}_{\beth_{2}}(\widetilde{U})\right\}, \min \left\{\mathfrak{K}_{\unlhd_{1}}(\widetilde{U}), \mathfrak{K}_{\beth_{2}}(\widetilde{U})\right\}\right\rangle: \widetilde{U} \in \check{Y}\right\}$.

(5) $\quad \hat{\beth}_{1} \widetilde{\sqcap} \hat{\beth}_{2}=\left\{\left\langle\widetilde{\mathbb{U}}, \min \left\{\breve{\Theta}_{\mathfrak{\beth}_{1}}(\widetilde{\mathbb{U}}), \breve{\Theta}_{\mathfrak{\beth}_{2}}(\widetilde{\mathbb{U}})\right\}, \max \left\{\mathfrak{K}_{\beth_{1}}(\widetilde{\mathbb{U}}), \mathfrak{K}_{\beth_{2}}(\widetilde{\mathbb{U}})\right\}\right\rangle: \mathbb{U} \in \check{Y}\right\}$.

(6) $\quad \hat{\beth}_{1}+\hat{\beth}_{2}=\left\{\left\langle\widetilde{U},\left(\breve{\Theta}_{\beth_{1}}^{\mathfrak{q}}(\widetilde{\mathbb{U}})+\breve{\Theta}_{\beth_{2}}^{\mathfrak{q}}(\widetilde{\mathbb{U}})-\breve{\Theta}_{\beth_{1}}^{\mathfrak{q}}(\widetilde{\mathbb{U}}) \breve{\Theta}_{\beth_{\beth}}^{\mathfrak{q}}(\widetilde{\mathbb{U}})\right)^{1 / \hat{\mathfrak{q}}}, \mathfrak{K}_{\beth_{1}}(\widetilde{\mathbb{U}}) \mathfrak{K}_{\beth_{2}}(\widetilde{U})\right\rangle: \mathbb{U} \in \check{Y}\right\}$.

(7) $\quad \hat{\beth}_{1} \cdot \hat{\beth}_{2}=\left\{\left\langle\widetilde{\mathbb{U}},\left(\breve{\Theta}_{\beth_{1}}(\widetilde{\mathbb{U}}) \breve{\Theta}_{\beth_{2}}(\widetilde{U}), \mathfrak{K}_{\beth_{1}}^{\mathfrak{q}}(\widetilde{U})+\mathfrak{K}_{\beth_{2}}^{\mathfrak{q}}(\widetilde{U})-\mathfrak{K}_{\beth_{1}}^{\mathfrak{q}}(\widetilde{\mathbb{U}}) \breve{\Theta}_{\beth_{2}}^{\mathfrak{q}}(\widetilde{U})\right)^{1 / \hat{\mathfrak{q}}}\right\rangle: \widetilde{U} \in \check{Y}\right\}$.

(8) $\alpha \hat{\beth_{1}}=\left\{\left\langle\widetilde{\cup},\left(1-\left(1-\breve{\Theta}_{\beth_{1}}(\widetilde{\mathbb{U}})^{\mathfrak{q}}\right)^{\alpha}\right)^{1 / \mathfrak{\mathfrak { q }}}, \mathfrak{K}_{\beth_{1}}(\widetilde{\mathbb{U}})^{\alpha}\right\rangle\right\}$.

(9) $\quad \hat{\beth}_{1}^{\alpha}=\left\{\left\langle\widetilde{\cup}, \breve{\Theta}_{\mathfrak{\beth}_{1}}(\widetilde{\mathbb{U}})^{\alpha},\left(1-\left(1-\mathfrak{K}_{\mathfrak{\beth}_{1}}^{\mathfrak{q}}(\widetilde{\mathbb{U}})\right)^{\alpha}\right)^{1 / \mathfrak{q}}\right\rangle\right\}$.

2.2. Operational Laws of $\mathfrak{q}-R O F N s$

Let $\widetilde{\Xi}_{1}=\left\langle\breve{\Theta}_{1}, \mathfrak{K}_{1}\right\rangle$ and $\widetilde{\Xi}_{2}=\left\langle\breve{\Theta}_{2}, \mathfrak{K}_{2}\right\rangle$ be $\mathfrak{q}$-ROFNs on a $\check{Y}$ [45]. Then

(1) $\overline{\widetilde{\Xi}_{1}}=\left\langle\mathfrak{K}_{1}, \breve{\Theta}_{1}\right\rangle$

(2) $\widetilde{\Xi}_{1} \vee \widetilde{\Xi}_{2}=\left\langle\max \left\{\breve{\Theta}_{1}, \breve{\Theta}_{2}\right\}, \min \left\{\mathfrak{K}_{1}, \mathfrak{K}_{2}\right\}\right\rangle$

(3) $\widetilde{\Xi}_{1} \wedge \widetilde{\Xi}_{2}=\left\langle\min \left\{\breve{\Theta}_{1}, \breve{\Theta}_{2}\right\}, \max \left\{\mathfrak{K}_{1}, \mathfrak{K}_{2}\right\}\right\rangle$

(4) $\widetilde{\Xi}_{1} \oplus \widetilde{\Xi}_{2}=\left\langle\left(\breve{\Theta}_{1}^{\mathfrak{q}}+\breve{\Theta}_{2}^{\mathfrak{q}}-\breve{\Theta}_{1}^{\mathfrak{q}} \breve{\Theta}_{2}^{\mathfrak{q}}\right)^{1 / \mathfrak{q}}, \mathfrak{K}_{1} \mathfrak{K}_{2}\right\rangle$

(5) $\quad \widetilde{\Xi}_{1} \otimes \widetilde{\Xi}_{2}=\left\langle\left(\breve{\Theta}_{1} \breve{\Theta}_{2},\left(\mathfrak{K}_{1}^{\mathfrak{q}}+\mathfrak{K}_{2}^{\mathfrak{q}}-\mathfrak{K}_{1}^{\mathfrak{q}} \mathfrak{K}_{2}^{\mathfrak{q}}\right)^{1 / \mathfrak{q}}\right\rangle\right.$ 
(6) $\alpha \widetilde{\Xi}_{1}=\left\langle\left(1-\left(1-\breve{\Theta}_{1}^{\mathfrak{q}}\right)^{\alpha}\right)^{1 / \mathfrak{q}}, \mathfrak{K}_{1}^{\alpha}\right\rangle$

(7) $\quad \widetilde{\Xi}_{1}^{\alpha}=\left\langle\breve{\Theta}_{1}^{\alpha}, 1-\left(\left(1-\mathfrak{K}_{1}^{\mathfrak{q}}\right)^{\alpha}\right)^{1 / \hat{\mathfrak{q}}}\right\rangle$

Definition 5 ([45]). Let $\widetilde{\Xi}_{\mathfrak{i}}=\left\langle\breve{\Theta}_{\mathfrak{i}}, \mathfrak{K}_{\mathfrak{i}}\right\rangle, \mathfrak{i}=(1, \ldots, \mathfrak{n})$ is a set of $\mathfrak{\mathfrak { q }}-R O F N$ s with weight vector $\hat{\omega}=\left(\hat{\omega}_{1}, \hat{\omega}_{2}, \ldots, \hat{\omega}_{\mathfrak{n}}\right)$ such that $\hat{\omega}_{\mathfrak{i}} \in[0,1]$ and $\sum_{\mathfrak{i}=1}^{\mathfrak{n}} \hat{\omega}_{\mathfrak{i}}=1$. The ( $\dot{\mathfrak{q}}-$ ROFWG) operator is

$$
\mathfrak{q}-\operatorname{ROFWG}\left(\widetilde{\Xi}_{1}, \widetilde{\Xi}_{2}, \ldots, \widetilde{\Xi}_{\mathfrak{n}}\right)=\left(\widetilde{\prod}_{\mathfrak{k}=1}^{\mathfrak{n}} \breve{\Theta}_{\mathfrak{k}}^{\hat{\omega}_{\mathfrak{k}}}, \sqrt[\mathfrak{q}]{1-\widetilde{\prod}_{\mathfrak{k}=1}^{\mathfrak{n}}\left(1-\mathfrak{K}_{\mathfrak{k}}^{\mathfrak{q}}\right) \hat{\boldsymbol{\omega}}_{\mathfrak{k}}}\right)
$$

Definition 6 ([28]). Suppose $\widetilde{\Xi}=\langle\breve{\Theta}, \mathfrak{K}\rangle$ is a $\mathfrak{\mathfrak { q }}-R O F N$. The score function $\widetilde{\top}$ of $\widetilde{\Xi}$ is determined as,

$$
\widetilde{\neg}(\widetilde{\Xi})=\breve{\Theta}^{q}-\mathfrak{K}^{q}
$$

$\widetilde{\neg}(\widetilde{\Xi}) \in[-1,1]$. The ranking of $\mathfrak{q}-R O F N$ s is described by score function. Large value of score function specifies high preference of $\mathfrak{q}$-ROFN. Although, score function is not efficient in several instances of $\mathfrak{q}-R O F N$. As for example, suppose $\widetilde{\Xi}_{1}=\langle 0.6138,0.2534\rangle$ and $\widetilde{\Xi}_{2}=\langle 0.7147,0.4453\rangle$ are two $\mathfrak{q}-R O F N$ s. Consider $q=2$, then $\widetilde{\urcorner}\left(\widetilde{\Xi}_{1}\right)=0.3125=\widetilde{\urcorner}\left(\widetilde{\Xi}_{2}\right)$ i.e., score functions of $\widetilde{\Xi}_{1}$ and $\widetilde{\Xi}_{2}$ are same. While comparing the $\mathfrak{q}-R O F N$, there is no need to only depend on the score function. To solve this problem, there is another approach, the accuracy function.

Definition 7 ([28]). Suppose $\widetilde{\Xi}=\langle\breve{\Theta}, \mathfrak{K}\rangle$ is a $\mathfrak{q}-R O F N$. An accuracy function $\mathfrak{S}$ of $\widetilde{\Xi}$ is determined as

$$
\mathfrak{S}(\widetilde{\Xi})=\breve{\Theta}^{\mathfrak{q}}+\mathfrak{K}^{\mathfrak{q}}
$$

$\mathfrak{S}(\widetilde{\Xi}) \in[0,1]$. The large value of accuracy function $\mathfrak{S}(\widetilde{\mathbb{\Xi}})$, determines high priorities of $\mathfrak{\mathfrak { q }}-R O F N$. For the above example, their accuracy functions are $\mathfrak{S}\left(\widetilde{\Xi}_{1}\right)=0.4409$ and $\mathfrak{S}\left(\widetilde{\Xi}_{2}\right)=0.7090$, so by the accuracy function we have $\widetilde{\Xi}_{1}<\widetilde{\Xi}_{2}$.

Definition 8. Let $\widetilde{\Xi}_{1}=\left\langle\breve{\Theta}_{1}, \mathfrak{K}_{1}\right\rangle$ and $\widetilde{\Xi}_{2}=\left\langle\breve{\Theta}_{2}, \mathfrak{K}_{2}\right\rangle$ are any two $\mathfrak{q}-R O F N s, \widetilde{\neg}\left(\widetilde{\Xi}_{1}\right), \widetilde{\urcorner}\left(\widetilde{\Xi}_{2}\right)$ are the score function of $\widetilde{\Xi}_{1}$ and $\widetilde{\Xi}_{2}$ and $\mathfrak{S}\left(\widetilde{\Xi}_{1}\right), \mathfrak{S}\left(\widetilde{\Xi}_{2}\right)$ are the accuracy functions of $\widetilde{\Xi}_{1}$ and $\widetilde{\Xi}_{2}$, respectively. Then

(1) If $\widetilde{\sim}\left(\widetilde{\Xi}_{1}\right)>\widetilde{\top}\left(\widetilde{\Xi}_{2}\right)$, then $\widetilde{\Xi}_{1}>\widetilde{\Xi}_{2}$.

(2) If $\widetilde{\urcorner}\left(\widetilde{\Xi}_{1}\right)=\widetilde{\urcorner}\left(\widetilde{\Xi}_{2}\right)$, then

(1) If $\mathfrak{S}\left(\widetilde{\Xi}_{1}\right)>\mathfrak{S}\left(\widetilde{\Xi}_{2}\right)$ then $\widetilde{\Xi}_{1}>\widetilde{\Xi}_{2}$.

(2) If $\mathfrak{S}\left(\widetilde{\Xi}_{1}\right)=\mathfrak{S}\left(\widetilde{\Xi}_{2}\right)$, then $\widetilde{\Xi}_{1}=\widetilde{\Xi}_{2}$.

\section{3. q́-ROF Information Under Generalized Parameter}

Suppose in a medical diagnosis, a patient is suffering an anonymous disease and provide his/her inclinations as $\mathfrak{q}$-ROFNs regarding symptoms $\mathfrak{E}=\left\{\mathfrak{h}_{1}, \mathfrak{h}_{2}, \mathfrak{h}_{3}\right\}$, where

(1) $\mathfrak{h}_{1}=$ Dry Cough (DC);

(2) $\mathfrak{h}_{2}=$ High Fever (HF);

(3) $\mathfrak{h}_{3}=$ Sore Throat (ST).

Let the $\mathfrak{q}-R O F S, \mathfrak{P}=\left\{(0.23,0.67)_{D C},(0.42,0.77)_{H F},(0.78,0.55)_{S T}\right\}(\mathfrak{\mathfrak { q }}=3)$ represents the preferences of the patient. The collected information is entirely based on his/her understanding, physical conditions and awareness in reporting the symptoms. Thereby, doctors treat the patient as a result of his presentation of symptoms, this may cause an imprecise outcome and patient might not be recovered according to data presented by a patient is not confirmed by one more doctor. Therefore, it is necessary to demonstrate the presented data to make the method quite similar to the situation of a patient. It can be obtained by introducing the idea of general parameter in the initial information, 
which indicates the confidence of an expert in conviction of the presented data to make the method very close to the actual circumstances. When a patient provided his/her preferences and is additionally evaluated by a physician/senior doctor who presents his/her data as $h=(0.5,0.4), \mathfrak{q}$-ROFS under generalized parameter (GP) is a

$$
\mathfrak{P}^{G}=\left\{(0.23,0.67)_{D C},(0.42,0.77)_{H F},(0.78,0.55)_{S T}(\mathbf{0 . 4 1}, \mathbf{0 . 8 4})\right\} \quad(\mathfrak{q}=3)
$$

Here, the indication of GP in bold is a q́-ROFN which diminish the inaccurate demonstration of imprecise data across the system of knowledge representation. The GP value capable of providing optimum solution of upgrading existing systems of decision experts, making sure a better accuracy in crucial decisions. The prior evaluation remains imprecise without the GP, which demonstrates that effectiveness of evaluation is uncertain. Whereby, in the information mapping system, the chances of substantial deformations of vague information can be discarded on the basis of judgment of a particular observer through another expert's opinion (in form of GP) in implementing the original $\mathfrak{q}$-ROPFNs. Consequently, the generalized $\mathfrak{q}$-rung orthopair FS (GQROFS) is defined as

Definition 9. Let $\check{Y}$ be a set of universal elements, a generalized $\mathfrak{q}$-rung orthopair FS (GQROFS) is of the form

$$
\mathfrak{G}=\left\{\left(\left\langle\widetilde{\cup}, \breve{\Theta}_{\mathfrak{G}}(\widetilde{\mathbb{U}}), \mathfrak{K}_{\mathfrak{G}}(\widetilde{U})\right\rangle\left(\breve{\Theta}_{\mathfrak{g}}, \mathfrak{K}_{\mathfrak{g}}\right)\right): \widetilde{U} \in \check{Y}\right\}
$$

with the condition that $0 \leq \breve{\Theta}_{\mathfrak{G}}^{\mathfrak{q}}(\widetilde{\cup})+\mathfrak{K}_{\mathfrak{G}}^{\mathfrak{q}}(\widetilde{\cup}) \leq 1,(\mathfrak{\mathfrak { q }} \geq 1)$ where, $\breve{\Theta}_{\mathfrak{G}}(\widetilde{\cup}): \check{Y} \rightarrow[0,1]$ indicates the degree of truthness and $\mathfrak{K}_{\mathfrak{G}}(\widetilde{\cup}): \check{Y} \rightarrow[0,1]$ indicates the degree of falsity of an alternative $\widetilde{U} \in \bar{Y}$. Here $\left(\breve{\Theta}_{\breve{\mathfrak{g}}}, \mathfrak{K}_{\mathfrak{\mathfrak { g }}}\right)$ is said to be GP which is a $\mathfrak{q}-R O F N$ indicated by other observer/decision maker signifying the preferable evaluation.

\section{4. q́-ROF Geometric Aggregation Operator Under Generalized Parameter}

In the presented section we introduce some geometric aggregation operators under generalized parameter, including the generalized q́ -rung orthopair fuzzy weighted geometric (GQROFWG) operator, generalized q́q-rung orthopair fuzzy ordered weighted geometric (GQROFOWG) operator, and generalized $\mathfrak{q}$-rung orthopair fuzzy hybrid geometric aggregation (GQROFHG) operator.

\subsection{The Generalized $\mathfrak{q}$-ROF Weighted Geometric Operator}

Definition 10. Let $\breve{\mathfrak{g}}=\left(\breve{\Theta}_{\breve{\mathfrak{g}}}, \mathfrak{K}_{\breve{\mathfrak{g}}}\right)$ be the GP for the $\mathfrak{\mathfrak { q }}-R O F N s \widetilde{\Xi}_{\mathfrak{i}}=\left(\breve{\Theta}_{\mathfrak{i}}, \mathfrak{K}_{\mathfrak{i}}\right)(\mathfrak{i}=1,2, \ldots, \mathfrak{n})$, then the GQROFWG-operator is determined as,

$$
\operatorname{GQROFWG}\left(\left(\widetilde{\Xi}_{1}, \widetilde{\Xi}_{2}, \ldots, \widetilde{\Xi}_{\mathfrak{n}}, \check{\mathfrak{g}}\right)\right)=\check{\mathfrak{g}} \otimes \mathfrak{q}-\operatorname{ROFWG}\left(\widetilde{\Xi}_{1}, \widetilde{\Xi}_{2}, \ldots, \widetilde{\Xi}_{\mathfrak{n}}\right)
$$

Theorem 11. Let $\widetilde{\Xi}_{\mathfrak{i}}=\left(\breve{\Theta}_{\mathfrak{i}}, \mathfrak{K}_{\mathfrak{i}}\right)(\mathfrak{i}=1,2, \ldots, \mathfrak{n})$ be a set of $\mathfrak{\mathfrak { q }}-R O F N$ s and $\hat{\omega}=\left(\hat{\omega}_{1}, \hat{\omega}_{2}, \ldots, \hat{\omega}_{\mathfrak{n}}\right)^{T}$ is a weight vector of $\widetilde{\Xi}_{\mathfrak{i}}$ such that $\hat{\omega}_{\mathfrak{i}} \in[0,1]$ and $\sum_{\mathfrak{i}=1}^{\mathfrak{n}} \hat{\omega}_{\mathfrak{i}}=1$. The GP is $\check{\mathfrak{g}}=\left(\breve{\Theta}_{\mathfrak{\mathfrak { g }}}, \mathfrak{K}_{\mathfrak{\mathfrak { g }}}\right)$, then the GQROFWG-operator is determined as

$$
\begin{aligned}
\operatorname{GQROFWG}\left(\left(\widetilde{\Xi}_{1}, \widetilde{\Xi}_{2}, \ldots, \widetilde{\Xi}_{\mathfrak{n}}\right), \check{\mathfrak{g}}\right) & =\check{\mathfrak{g}} \otimes \mathfrak{q}-R O F W G\left(\widetilde{\Xi}_{1}, \widetilde{\Xi}_{2}, \ldots, \widetilde{\Xi}_{\mathfrak{n}}\right) \\
& =\left(\sqrt[\mathfrak{q}]{\left(\breve{\Theta}_{\mathfrak{g}}\right)^{\mathfrak{q}}+\left(1-\left(\breve{\Theta}_{\mathfrak{\mathfrak { g }}}\right)^{\mathfrak{q}}\right) \widetilde{\prod}_{\mathfrak{i}=1}^{\mathfrak{n}}\left(\breve{\Theta}_{\mathfrak{i}}^{\hat{\omega}_{\mathfrak{i}}}\right) \mathfrak{\mathfrak { q }}}, \mathfrak{K}_{\mathfrak{\mathfrak { g }}} \cdot \sqrt[\mathfrak{q}]{1-\widetilde{\prod}_{\mathfrak{i}=1}^{\mathfrak{n}}\left(1-\left(\mathfrak{K}_{\mathfrak{i}}\right) \mathfrak{q}\right)^{\hat{\omega}_{\mathfrak{i}}}}\right)
\end{aligned}
$$

Proof. We use mathematical induction.

For $\mathfrak{n}=2$,

$$
\operatorname{GQROFWG}\left(\left(\widetilde{\Xi}_{1}, \widetilde{\Xi}_{2}\right), \check{\mathfrak{g}}\right)=\check{\mathfrak{g}} \otimes\left(\widetilde{\Xi}_{1}^{\hat{\omega}_{1}} \otimes \widetilde{\Xi}_{2}^{\hat{\omega}_{2}}\right)
$$


First we solve $\left(\widetilde{\Xi}_{1}^{\hat{\omega}_{1}} \otimes \widetilde{\Xi}_{2}^{\hat{\omega}_{2}}\right)$, by using the operational law of $\mathfrak{q}-R O F S$, we have

$$
\begin{aligned}
\widetilde{\Xi}_{1}^{\hat{\omega}_{1}} \otimes \widetilde{\Xi}_{2}^{\hat{\omega}_{2}} & =\left(\breve{\Theta}_{1}, \mathfrak{K}_{1}\right)^{\hat{\omega}_{1}} \otimes\left(\breve{\Theta}_{2}, \mathfrak{K}_{2}\right)^{\hat{\omega}_{2}} \\
& =\left(\breve{\Theta}_{1}^{\hat{\omega}_{1}}, \sqrt[\mathfrak{q}]{1-\left(1-\mathfrak{K}_{1}^{\mathfrak{q}}\right)^{\hat{\omega}_{1}}} \otimes \breve{\Theta}_{2}^{\hat{\omega}_{2}}, \sqrt[\mathfrak{q}]{1-\left(1-\mathfrak{K}_{2}^{\mathfrak{q}}\right)^{\hat{\omega}_{2}}}\right) \\
& =\left(\breve{\Theta}_{1}^{\hat{\omega}_{1}} \cdot \breve{\Theta}_{2}^{\hat{\omega}_{2}}, \sqrt[\mathfrak{q}]{1-\left(1-\mathfrak{K}_{1}^{\mathfrak{q}}\right)}\right)^{\hat{\omega}_{1}} \cdot\left(1-\mathfrak{K}_{2}^{\mathfrak{q}}\right)^{\hat{\omega}_{2}}
\end{aligned}
$$

Now,

$$
\begin{aligned}
& \check{\mathfrak{g}} \otimes\left(\widetilde{\Xi}_{1}^{\hat{\omega}_{1}} \otimes \widetilde{\Xi}_{2}^{\hat{\omega}_{2}}\right)=\left(\breve{\Theta}_{\mathfrak{g}}, \mathfrak{K}_{\mathfrak{g}}\right) \otimes\left(\breve{\Theta}_{1}^{\hat{\omega}_{1}} \cdot \breve{\Theta}_{2}^{\hat{\omega}_{2}}, \sqrt[\mathfrak{q}]{1-\left(1-\mathfrak{K}_{1}^{\mathfrak{q}}\right)^{\hat{\omega}_{1}} \cdot\left(1-\mathfrak{K}_{2}^{\mathfrak{q}}\right)^{\hat{\omega}_{2}}}\right) \\
& =\left(\sqrt[\mathfrak{q}]{\left(\breve{\Theta}_{\mathfrak{\mathfrak { g }}}\right)^{\mathfrak{q}}+\left(\breve{\Theta}_{1}^{\hat{\omega}_{1}} \breve{\Theta}_{2}^{\hat{\omega}_{2}}\right)^{\mathfrak{q}}-\left(\breve{\Theta}_{\breve{\mathfrak{g}}}\right)^{\mathfrak{q}} \cdot\left(\breve{\Theta}_{1}^{\hat{\omega}_{1}} \cdot \breve{\Theta}_{2}^{\hat{\omega}_{2}}\right)^{\mathfrak{q}}}, \mathfrak{K}_{\mathfrak{\mathfrak { g }}} \cdot \sqrt[\mathfrak{q}]{1-\left(1-\mathfrak{K}_{1}^{\mathfrak{q}}\right)^{\hat{\omega}_{1}} \cdot\left(1-\mathfrak{K}_{2}^{\mathfrak{q}}\right)^{\hat{\omega}_{2}}}\right) \\
& =\left(\sqrt[\mathfrak{q}]{\left(\check{\Theta}_{\mathfrak{g}}\right)^{\mathfrak{q}}+\left(1-\left(\check{\Theta}_{\mathfrak{\mathfrak { g }}}\right) \mathfrak{\mathfrak { q }}\right) \cdot\left(( \check { \Theta } _ { 1 } ^ { \hat { \omega } _ { 1 } } ) \mathfrak { \mathfrak { q } } \left(\left(\breve{\Theta}_{2}^{\tilde{\omega}_{2}}\right)^{\mathfrak{q}}\right.\right.}, \mathfrak{K}_{\mathfrak{\mathfrak { g }}} \cdot \sqrt[\mathfrak{q}]{1-\left(1-\mathfrak{K}_{1}^{\mathfrak{q}}\right) \hat{\omega}_{1} \cdot\left(1-\mathfrak{K}_{2}^{\mathfrak{q}}\right)^{\hat{\omega}_{2}}}\right) \\
& \operatorname{GQROFWG}\left(\left(\widetilde{\Xi}_{1}, \widetilde{\Xi}_{2}\right), \mathfrak{g}\right)=\left(\sqrt[\mathfrak{q}]{\left(\breve{\Theta}_{\mathfrak{g}}\right)^{\mathfrak{q}}+\left(1-\left(\breve{\Theta}_{\mathfrak{\mathfrak { g }}}\right)^{\mathfrak{q}}\right) \cdot \widetilde{\prod}_{\mathfrak{i}=1}^{2}\left(\left(\breve{\Theta}_{\mathfrak{i}}^{\hat{\omega}_{\mathfrak{i}}}\right)^{\mathfrak{q}}\right.}, \mathfrak{K}_{\mathfrak{g}} \cdot \sqrt[\mathfrak{q}]{1-\widetilde{\prod}_{i=1}^{2}\left(1-\mathfrak{K}_{\mathfrak{i}}^{\mathfrak{\mathfrak { q }}}\right)^{\hat{\omega}_{\mathfrak{i}}}}\right)
\end{aligned}
$$

For $\mathfrak{n}=2$, result is true.

Suppose that result satisfied for $\mathfrak{n}=\mathfrak{k}$,

$$
\begin{aligned}
\operatorname{GQROFWG}\left(\left(\widetilde{\Xi}_{1}, \widetilde{\Xi}_{2}, \ldots, \widetilde{\Xi}_{\mathfrak{n}}\right), \check{\mathfrak{g}}\right) & =\check{\mathfrak{g}} \otimes \mathfrak{q}-\operatorname{ROFWG}\left(\widetilde{\Xi}_{1}, \widetilde{\Xi}_{2}, \ldots, \widetilde{\Xi}_{\mathfrak{n}}\right) \\
& =\left(\sqrt[\mathfrak{q}]{\left(\check{\Theta}_{\mathfrak{\mathfrak { g }}}\right)^{\mathfrak{q}}+\left(1-\left(\check{\Theta}_{\mathfrak{\mathfrak { g }}}\right) \mathfrak{q}\right) \widetilde{\prod}_{i=1}^{\mathfrak{k}}\left(\check{\Theta}_{\mathfrak{i}}^{\hat{\omega}_{\mathfrak{i}}}\right)^{\mathfrak{q}},}, \mathfrak{K}_{\mathfrak{g}} \cdot \sqrt[\mathfrak{q}]{1-\widetilde{\prod}_{\mathfrak{i}=1}^{\mathfrak{k}}\left(1-\left(\mathfrak{K}_{\mathfrak{i}}\right)^{\mathfrak{q}}\right)^{\hat{\mathfrak{i}} \mathfrak{i}}}\right)
\end{aligned}
$$

Now we will prove for $\mathfrak{n}=\mathfrak{k}+1$,

$$
\begin{aligned}
& \operatorname{GQROFWG}\left(\left(\widetilde{\Xi}_{1}, \widetilde{\Xi}_{2}, \ldots, \widetilde{\Xi}_{\mathfrak{k}}, \widetilde{\Xi}_{\mathfrak{k}+1}\right), \breve{\mathfrak{g}}\right)=\breve{\mathfrak{g}} \otimes\left(\widetilde{\Xi}_{1}^{\hat{\omega}_{1}} \otimes \ldots, \otimes \widetilde{\Xi}_{\mathfrak{k}}^{\hat{\omega}_{\mathfrak{k}}} \otimes \widetilde{\Xi}_{\mathfrak{k}+1}^{\hat{\omega}_{\mathfrak{k}}+1}\right) \\
& =\left(\sqrt[\mathfrak{q}]{\left(\breve{\Theta}_{\breve{\mathfrak{g}}}\right)^{\mathfrak{q}}+\left(1-\left(\check{\Theta}_{\mathfrak{\mathfrak { g }}}\right)^{\mathfrak{q}}\right)\left(\left(\check{\Theta}_{\mathfrak{k}+1}\right)^{\hat{\mathfrak{\omega}}_{\mathfrak{k}+1}}\right)^{\mathfrak{q}} \widetilde{\prod}_{\mathfrak{i}=1}^{\mathfrak{k}}\left(\breve{\Theta}_{\mathfrak{i}}^{\hat{\boldsymbol{\omega}}_{\mathfrak{i}}}\right)^{\mathfrak{q}}},\right. \\
& \mathfrak{K}_{\mathfrak{\mathfrak { g }}} \cdot \sqrt[\mathfrak{q}]{\left.1-\left(1-\left(\mathfrak{K}_{\mathfrak{k}+1}\right)^{\mathfrak{q}}\right)^{\hat{\omega}_{\mathfrak{k}+1}} \widetilde{\prod}_{\mathfrak{i}=1}^{\mathfrak{k}}\left(1-\left(\mathfrak{K}_{\mathfrak{i}}\right)^{\mathfrak{q}}\right)^{\hat{\omega}_{\mathfrak{i}}}\right)}
\end{aligned}
$$

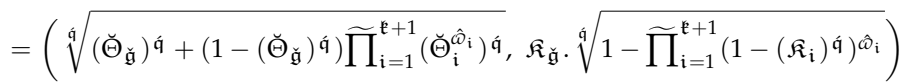

The result is true for $\mathfrak{n}=\mathfrak{k}+1$. Consequently, the result holds, under generalized parameter for any number.

Theorem 12. By using GQROFWG-operator, the aggregated value is also a $\mathfrak{q}-R O P F N$.

Proof. For every $\mathfrak{i}=1,2, \ldots, \mathfrak{n}$, we have $0 \leq \breve{\Theta}_{\mathfrak{i}}, \mathfrak{K}_{\mathfrak{i}} \leq 1$ and $0 \leq \breve{\Theta}_{\mathfrak{i}}^{\mathfrak{q}}+\mathfrak{K}_{\mathfrak{i}}^{\mathfrak{q}} \leq 1,(\mathfrak{\mathfrak { q }} \geq 1)$ implies that $0 \leq 1-\mathfrak{K}_{\mathfrak{i}}^{\mathfrak{q}} \leq 1$. Therefore,

$$
\begin{aligned}
& 0 \leq \widetilde{\prod}_{\mathfrak{i}=1}^{\mathfrak{n}}\left(1-\mathfrak{K}_{\mathfrak{i}}^{\mathfrak{q}}\right)^{\hat{\omega}_{\mathfrak{i}}} \leq 1 \\
& 0 \leq \mathfrak{K}_{\mathfrak{g}} \cdot \sqrt[\mathfrak{q}]{1-\widetilde{\prod}_{\mathfrak{i}=1}^{\mathfrak{n}}\left(1-\mathfrak{K}_{\mathfrak{i}}^{\mathfrak{q}}\right)^{\hat{\omega}_{\mathfrak{i}}}} \leq 1 \text { for } \quad 0 \leq \mathfrak{K}_{\mathfrak{\mathfrak { g }}} \leq 1 .
\end{aligned}
$$

In addition, for $0 \leq \breve{\Theta}_{\breve{\mathfrak{g}}} \leq 1$, one can write, $0 \leq \sqrt[\mathfrak{q}]{\left(\breve{\Theta}_{\breve{\mathfrak{g}}}\right)^{\mathfrak{q}}+\left(1-\left(\breve{\Theta}_{\mathfrak{\mathfrak { g }}}\right)^{\mathfrak{q}}\right) \widetilde{\Pi}_{\mathfrak{i}=1}^{\mathfrak{n}}\left(\breve{\Theta}_{\mathfrak{i}}^{\hat{\omega}_{\mathfrak{i}}}\right)^{\mathfrak{q}}} \leq 1$. 
Now,

$$
\begin{aligned}
& =\left(\sqrt[\mathfrak{q}]{\left(\breve{\Theta}_{\breve{\mathfrak{g}}}\right)^{\mathfrak{q}}+\left(1-\left(\breve{\Theta}_{\breve{\mathfrak{g}}}\right) \mathfrak{q}\right) \widetilde{\prod}_{\mathfrak{i}=1}^{\mathfrak{n}}\left(\breve{\Theta}_{\mathfrak{i}}^{\hat{\omega}_{\mathfrak{i}}}\right)^{\mathfrak{q}}}\right)^{\mathfrak{q}}+\left(\mathfrak{K}_{\mathfrak{g}} \cdot \sqrt[\mathfrak{q}]{1-\widetilde{\prod}_{\mathfrak{i}=1}^{\mathfrak{n}}\left(1-\left(\mathfrak{K}_{\mathfrak{i}}\right)^{\mathfrak{q}}\right)^{\hat{\omega}_{\mathfrak{i}}}}\right)^{\mathfrak{q}} \\
& =\left(\left(\breve{\Theta}_{\mathfrak{\mathfrak { g }}}\right)^{\mathfrak{q}}+\left(1-\left(\breve{\Theta}_{\mathfrak{\mathfrak { g }}}\right)^{\mathfrak{\mathfrak { q }}}\right) \widetilde{\prod}_{\mathfrak{i}=1}^{\mathfrak{n}}\left(\breve{\Theta}_{\mathfrak{i}}^{\hat{\omega}_{\mathfrak{i}}}\right)^{\mathfrak{q}}\right)+\left(\mathfrak{K}_{\mathfrak{\mathfrak { g }}}\right)^{\mathfrak{\mathfrak { q }}}\left(1-\widetilde{\prod}_{\mathfrak{i}=1}^{\mathfrak{n}}\left(1-\left(\mathfrak{K}_{\mathfrak{i}}\right)^{\mathfrak{q}}\right)^{\hat{\omega}_{\mathfrak{i}}}\right) \\
& =\left(\left(\mathfrak{K}_{\mathfrak{g}}\right)^{\mathfrak{q}}+\left(\breve{\Theta}_{\breve{\mathfrak{g}}}\right)^{\mathfrak{q}}\right)+\widetilde{\prod}_{\mathfrak{i}=1}^{\mathfrak{n}}\left(\breve{\Theta}_{\mathfrak{i}}^{\hat{\omega}_{\mathfrak{i}}}\right)^{\mathfrak{q}}-\left(\mathfrak{K}_{\mathfrak{g}}\right)^{\mathfrak{q}} \widetilde{\prod}_{\mathfrak{i}=1}^{\mathfrak{n}}\left(1-\left(\mathfrak{K}_{\mathfrak{i}}\right)^{\mathfrak{q}}\right)^{\hat{\omega}_{\mathfrak{i}}}-\left(\breve{\Theta}_{\breve{\mathfrak{g}}}\right)^{\mathfrak{q}} \widetilde{\prod}_{\mathfrak{i}=1}^{\mathfrak{n}}\left(\breve{\Theta}_{\mathfrak{i}}^{\hat{\omega}_{\mathfrak{i}}}\right)^{\mathfrak{q}}
\end{aligned}
$$

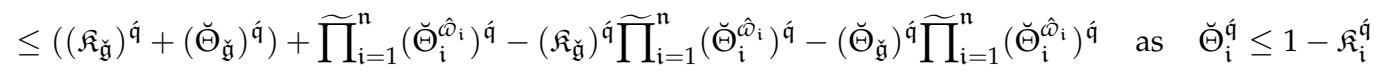

$$
\begin{aligned}
& \leq\left(\left(\mathfrak{K}_{\breve{\mathfrak{g}}}\right)^{\mathfrak{\mathfrak { q }}}+\left(\breve{\Theta}_{\breve{\mathfrak{g}}}\right)^{\mathfrak{q}}\right)+\widetilde{\prod}_{\mathfrak{i}=1}^{\mathfrak{n}}\left(\breve{\Theta}_{\mathfrak{i}}^{\hat{\omega}_{\mathfrak{i}}}\right)^{\mathfrak{q}}-\left(\left(\mathfrak{K}_{\breve{\mathfrak{g}}}\right)^{\mathfrak{q}}+\left(\breve{\Theta}_{\breve{\mathfrak{g}}}\right)^{\mathfrak{q}}\right) \widetilde{\prod}_{\mathfrak{i}=1}^{\mathfrak{n}}\left(\breve{\Theta}_{\mathfrak{i}}^{\hat{\omega}_{\mathfrak{i}}}\right)^{\mathfrak{q}} \\
& \leq\left(\left(\mathfrak{K}_{\mathfrak{g}}\right)^{\mathfrak{q}}+\left(\breve{\Theta}_{\mathfrak{g}}\right)^{\mathfrak{q}}\right)\left(1-\widetilde{\prod}_{\mathfrak{i}=1}^{\mathfrak{n}}\left(\breve{\Theta}_{\mathfrak{i}}^{\hat{\omega}_{\mathfrak{i}}}\right)^{\mathfrak{q}}\right)+\widetilde{\prod}_{\mathfrak{i}=1}^{\mathfrak{n}}\left(\breve{\Theta}_{\mathfrak{i}}^{\hat{\omega}_{\mathfrak{i}}}\right)^{\mathfrak{q}} \\
& \leq 1-\widetilde{\prod}_{i=1}^{\mathfrak{n}}\left(\breve{\Theta}_{i}^{\hat{\omega}_{i}}\right)^{\mathfrak{q}}+\widetilde{\prod}_{i=1}^{\mathfrak{n}}\left(\breve{\Theta}_{\mathfrak{i}}^{\hat{\omega}_{i}}\right)^{\mathfrak{q}} \leq 1
\end{aligned}
$$

Hence, the aggregated value obtained by the GQROFWG-operator is a q́-ROPFN.

Example 13. Consider $\breve{\mathfrak{g}}=(0.5,0.7)$ is a GP of four $\mathfrak{\mathfrak { q }}$-ROPFNs. $\widetilde{\Xi}_{1}=(0.23,0.67), \widetilde{\Xi}_{2}=(0.42,0.77)$, $\widetilde{\Xi}_{3}=(0.78,0.55)$ and $\widetilde{\Xi}_{4}=(0.41,0.84)$ with a weight vector $\hat{\boldsymbol{\omega}}=(0.1,0.2,0.3,0.4)$, here $\hat{\mathfrak{q}}=3$, then

$$
\sqrt[\mathfrak{q}]{\left(\breve{\Theta}_{\mathfrak{\mathfrak { g }}}\right)^{\mathfrak{q}}+\left(1-\left(\breve{\Theta}_{\mathfrak{\mathfrak { g }}}\right)^{\mathfrak{q}}\right) \widetilde{\prod}_{\mathfrak{i}=1}^{\mathfrak{n}}\left(\breve{\Theta}_{\mathfrak{i}}^{\hat{\omega}_{\mathfrak{i}}}\right)^{\mathfrak{q}}}=0.60071
$$

In addition,

$$
\mathfrak{K}_{\mathfrak{\mathfrak { g }}} \cdot \sqrt[\mathfrak{q}]{1-\widetilde{\prod}_{\mathfrak{i}=1}^{\mathfrak{n}}\left(1-\left(\mathfrak{K}_{\mathfrak{i}}\right)^{\mathfrak{q}}\right)^{\hat{\omega}_{\mathfrak{i}}}}=0.53054
$$

By Theorem 3.2, we have

$$
\begin{aligned}
\operatorname{GQROFWG}\left(\left(\widetilde{\Xi}_{1}, \widetilde{\Xi}_{2}, \widetilde{\Xi}_{3}, \widetilde{\Xi}_{4}\right), \mathfrak{g}\right) & =\check{\mathfrak{g}} \otimes \mathfrak{\mathfrak { q }}-\operatorname{ROFWG}\left(\widetilde{\Xi}_{1}, \widetilde{\Xi}_{2}, \ldots, \widetilde{\Xi}_{\mathfrak{n}}\right) \\
& =\left(\sqrt[\mathfrak{q}]{\left(\breve{\Theta}_{\mathfrak{g}}\right)^{\mathfrak{q}}+\left(1-\left(\breve{\Theta}_{\mathfrak{g}}\right)^{\mathfrak{q}}\right) \widetilde{\prod}_{\mathfrak{i}=1}^{\mathfrak{k}}\left(\breve{\Theta}_{\mathfrak{i}}^{\hat{\omega}_{\mathfrak{i}}}\right)^{\mathfrak{q}},} \mathfrak{K}_{\mathfrak{g}} \cdot \sqrt[\mathfrak{q}]{1-\widetilde{\prod}_{\mathfrak{i}=1}^{\mathfrak{k}}\left(1-\left(\mathfrak{K}_{\mathfrak{i}}\right)^{\mathfrak{q}}\right)^{\hat{\mathfrak{\omega}}_{\mathfrak{i}}}}\right) \\
& =(0.60071,0.53054)
\end{aligned}
$$

Proposition 14. Let $\widetilde{\Xi}_{\mathfrak{i}}=\left(\breve{\Theta}_{\mathfrak{i}}, \mathfrak{K}_{\mathfrak{i}}\right)(\mathfrak{i}=1,2, \ldots, \mathfrak{n})$ be a set of $\mathfrak{\mathfrak { q }}-R O F N$ s and $\hat{\omega}=\left(\hat{\omega}_{1}, \hat{\omega}_{2}, \ldots, \hat{\omega}_{\mathfrak{n}}\right)^{T}$ is a weight vector of $\widetilde{\Xi}_{\mathfrak{i}}$ such that $\hat{\omega}_{\mathfrak{i}} \in[0,1]$ and $\sum_{\mathfrak{i}=1}^{\mathfrak{n}} \hat{\omega}_{\mathfrak{i}}=1$. Generalized parameter is $\breve{\mathfrak{g}}=\left(\breve{\Theta}_{\mathfrak{\mathfrak { g }}}, \mathfrak{K}_{\mathfrak{g}}\right)$, then the GQROFWG-operator has the following properties:

1. (Idempotency) If $\widetilde{\Xi}_{\mathfrak{i}}=\widetilde{\Xi}(\forall i=1,2, \ldots, \mathfrak{n})$, then

$$
\operatorname{GQROFWG}\left(\left(\widetilde{\Xi}_{1}, \widetilde{\Xi}_{2}, \ldots, \widetilde{\Xi}_{\mathfrak{n}}\right), \mathfrak{g}\right)=\check{\mathfrak{g}} \otimes \widetilde{\Xi}
$$

2. (Boundary condition) if $\widetilde{\Xi}_{\mathfrak{i}}^{-}=\left(\breve{\Theta}_{\mathfrak{\mathfrak { g }} \otimes}^{\min } \widetilde{\Xi}_{\mathfrak{i}}^{\prime}, \mathfrak{K}_{\mathfrak{\mathfrak { g }} \otimes}^{\max } \widetilde{\Xi}_{\mathfrak{i}}\right)$ and $\widetilde{\Xi}_{\mathfrak{i}}^{+}=\left(\breve{\Theta}_{\mathfrak{\mathfrak { g }} \otimes}^{\max } \widetilde{\Xi}_{\mathfrak{i}}, \mathfrak{K}_{\mathfrak{\mathfrak { g }} \otimes \widetilde{\Xi}_{\mathfrak{i}}}^{\min }\right)$, then for every $\hat{\omega}_{\mathfrak{i}}$,

$$
\widetilde{\Xi}_{\mathfrak{i}}^{-} \leq \operatorname{GQROFWG}\left(\left(\widetilde{\Xi}_{1}, \widetilde{\Xi}_{2}, \ldots, \widetilde{\Xi}_{\mathfrak{n}}\right), \check{\mathfrak{g}}\right) \leq \widetilde{\Xi}_{\mathfrak{i}}^{+}
$$

3. (Monotonicity) Let $\widetilde{\Xi}_{\mathfrak{i}}^{\star}=\left(\breve{\Theta}_{\mathfrak{i}}^{\star}, \mathfrak{K}_{\mathfrak{i}}^{\star}\right)(\mathfrak{i}=1,2, \ldots, \mathfrak{n})$ be a set of $\dot{\mathfrak{q}}-$ ROFNs such that $\breve{\Theta}_{\mathfrak{i}} \leq \breve{\Theta}_{\mathfrak{i}}^{\star}$ and $\mathfrak{K}_{\mathfrak{i}} \geq \mathfrak{K}_{\mathfrak{i}}^{\star}$ for all $i$, then for every $\hat{\omega}_{i}$,

$$
\operatorname{GQROFWG}\left(\left(\widetilde{\Xi}_{1}, \widetilde{\Xi}_{2}, \ldots, \widetilde{\Xi}_{\mathfrak{n}}\right), \mathfrak{g}\right) \leq \operatorname{GQROFWG}\left(\left(\widetilde{\Xi}_{1}^{\star}, \widetilde{\Xi}_{2}^{\star}, \ldots, \widetilde{\Xi}_{\mathfrak{n}}^{\star}\right), \check{\mathfrak{g}}\right)
$$


4. (Commutativity) Let $\widetilde{\Xi}_{\mathfrak{i}}=\left(\breve{\Theta}_{\mathfrak{i}}, \mathfrak{K}_{\mathfrak{i}}\right)(\mathfrak{i}=1,2, \ldots, \mathfrak{n})$ and $\widetilde{\Xi}_{\mathfrak{i}}^{*}=\left(\breve{\Theta}_{\mathfrak{i}}^{*}, \mathfrak{K}_{\mathfrak{i}}{ }^{*}\right)(\mathfrak{i}=1,2, \ldots, \mathfrak{n})$ be two sets of $\mathfrak{n}$ $\mathfrak{q}$-ROFNs such that $\widetilde{\Xi}_{\mathfrak{i}}^{*}$ is any permutation of $\widetilde{\Xi}_{\mathfrak{i}}$, then

$$
\operatorname{GQROFWG}\left(\left(\widetilde{\Xi}_{1}, \widetilde{\Xi}_{2}, \ldots, \widetilde{\Xi}_{\mathfrak{n}}\right), \check{\mathfrak{g}}\right)=\operatorname{GQROFWG}\left(\left(\widetilde{\Xi}_{1}^{*}, \widetilde{\Xi}_{2}^{*}, \ldots, \widetilde{\Xi}_{\mathfrak{n}}^{*}\right), \check{\mathfrak{g}}\right)
$$

Proof. 1. if $\widetilde{\Xi}_{\mathfrak{i}}=\widetilde{\Xi}(\forall i=1,2, \ldots, \mathfrak{n})$, then by GQROFWG-operator,

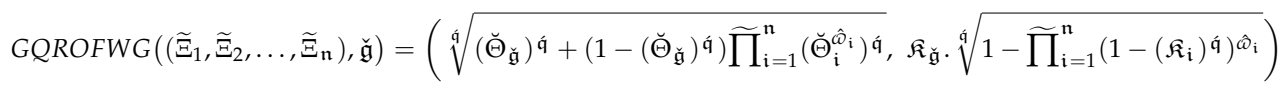

$$
\begin{aligned}
& =\left(\sqrt[\mathfrak{q}]{\left(\breve{\Theta}_{\mathfrak{g}}\right)^{\mathfrak{q}}+\left(1-\left(\breve{\Theta}_{\mathfrak{g}}\right)^{\mathfrak{q}}\right)\left(\breve{\Theta}^{\check{\Theta}_{\mathfrak{i}=1}^{n} \hat{\omega}_{\mathfrak{i}}}\right)^{\mathfrak{q}}}, \mathfrak{K}_{\mathfrak{\mathfrak { g }}} \cdot \sqrt[\mathfrak{q}]{1-\left(1-(\mathfrak{K})^{\mathfrak{q}}\right)^{\sum_{\mathfrak{i}=1}^{n} \hat{\omega}_{\mathfrak{i}}}}\right) \\
& =\left(\sqrt[\mathfrak{q}]{\left(\check{\Theta}_{\mathfrak{g}}\right)^{\mathfrak{q}}+\left(1-\left(\check{\Theta}_{\mathfrak{\mathfrak { g }}}\right)^{\mathfrak{q}}\right)(\check{\Theta})^{\mathfrak{q}}}, \mathfrak{K}_{\mathfrak{\mathfrak { g }}} \cdot \sqrt[\mathfrak{q}]{1-\left(1-(\mathfrak{K})^{\mathfrak{q}}\right)}\right) \\
& =\left(\sqrt[\mathfrak{q}]{\left(\breve{\Theta}_{\mathfrak{g}}\right) \mathfrak{q}+\check{\Theta}^{\mathfrak{q}}-\left(\breve{\Theta}_{\mathfrak{\mathfrak { g }}}\right) \mathfrak{q}(\check{\Theta}) \dot{\mathfrak{q}}}, \mathfrak{K}_{\mathfrak{\mathfrak { g }}} \cdot \mathfrak{K}\right) \\
& =\check{\mathfrak{g}} \otimes \widetilde{\Xi}
\end{aligned}
$$

2. Let $\widetilde{\Xi}_{\mathfrak{i}}^{-}=\left(\breve{\Theta}_{\mathfrak{\mathfrak { g }} \otimes \widetilde{\Xi}_{\mathfrak{i}}}^{\min }, \mathfrak{K}_{\mathfrak{\mathfrak { g }} \otimes}^{\max } \widetilde{\Xi}_{\mathfrak{i}}\right)$ and $\widetilde{\Xi}_{\mathfrak{i}}^{+}=\left(\breve{\Theta}_{\mathfrak{g} \otimes}^{\max }, \widetilde{\Xi}_{\mathfrak{i}} \mathfrak{K}_{\mathfrak{g} \otimes \widetilde{\mathfrak{\Xi}}_{\mathfrak{i}}}^{\min }\right)$, where $\mathfrak{K}_{\mathfrak{\mathfrak { g }} \otimes}^{\min } \widetilde{\Xi}_{\mathfrak{i}}=\mathfrak{K}_{\breve{\mathfrak{g}}}\left(\min \mathfrak{K}_{\mathfrak{i}}\right), \mathfrak{K}_{\mathfrak{\mathfrak { g }} \otimes}^{\max \widetilde{\Xi}_{\mathfrak{i}}}=$ $\mathfrak{K}_{\mathfrak{\mathfrak { g }}}\left(\max \mathfrak{K}_{\mathfrak{i}}\right), \mathfrak{K}_{\breve{\mathfrak{g}} \otimes \widetilde{\Xi}_{\mathfrak{i}}}^{\min }=\sqrt[\mathfrak{q}]{\breve{\Theta}_{\mathfrak{g}}^{\hat{q}}+\left(1-\breve{\Theta}_{\mathfrak{g}}^{\mathfrak{q}}\right)\left(\min \left(\breve{\Theta}_{\mathfrak{i}}\right)\right)^{\mathfrak{q}}}$, and $\mathfrak{K}_{\mathfrak{g} \otimes \breve{\Xi}_{\mathfrak{i}}}^{\max }=\sqrt[\mathfrak{q}]{\breve{\Theta}_{\mathfrak{g}}^{\mathfrak{q}}+\left(1-\breve{\Theta}_{\mathfrak{g}}^{\mathfrak{q}}\right)\left(\max \left(\breve{\Theta}_{\mathfrak{i}}\right)\right)^{\mathfrak{q}}}$ for all $\mathfrak{i}$, it is clear that $\min \left(\mathfrak{K}_{\mathfrak{i}}\right) \leq \mathfrak{K}_{\mathfrak{i}} \leq \max \left(\mathfrak{K}_{\mathfrak{i}}\right) \Rightarrow \max \left(1-\mathfrak{K}_{\mathfrak{i}}^{\mathfrak{q}}\right) \leq\left(1-\mathfrak{K}_{\mathfrak{i}}^{\mathfrak{q}}\right) \leq \min \left(1-\mathfrak{K}_{\mathfrak{i}}^{\mathfrak{q}}\right)$, for each $\hat{\omega}$,

$$
\begin{aligned}
& \Rightarrow \widetilde{\prod}_{\mathfrak{i}=1}^{\mathfrak{n}}\left(1-\max \left(\mathfrak{K}_{\mathfrak{i}}\right)^{\mathfrak{q}}\right)^{\hat{\omega}_{\mathfrak{i}}} \leq \widetilde{\prod}_{i=1}^{\mathfrak{n}}\left(1-\mathfrak{K}_{\mathfrak{i}}^{\mathfrak{q}}\right)^{\hat{\omega}_{\mathfrak{i}}} \leq \widetilde{\prod}_{i=1}^{\mathfrak{n}}\left(1-\min \left(\mathfrak{K}_{\mathfrak{i}}\right)^{\mathfrak{q}}\right)^{\hat{\omega}_{\mathfrak{i}}} \\
& \Rightarrow\left(1-\max \left(\mathfrak{K}_{\mathfrak{i}}\right)^{\mathfrak{q}}\right)^{\sum_{\mathfrak{i}=1}^{\mathfrak{n}} \hat{\omega}_{\mathfrak{i}}} \leq \widetilde{\Pi}_{\mathfrak{i}=1}^{\mathfrak{n}}\left(1-\mathfrak{K}_{\mathfrak{i}}^{\mathfrak{q}}\right)^{\hat{\omega}_{\mathfrak{i}}} \leq\left(1-\min \left(\mathfrak{K}_{\mathfrak{i}}\right)^{\mathfrak{q}}\right)^{\sum_{\mathfrak{i}=1}^{\mathfrak{n}} \hat{\omega}_{\mathfrak{i}}} \\
& \Rightarrow 1-\left(\left(1-\min \left(\mathfrak{K}_{\mathfrak{i}}\right)^{\mathfrak{q}}\right)\right) \leq \widetilde{\prod}_{\mathfrak{i}=1}^{\mathfrak{n}}\left(1-\mathfrak{K}_{\mathfrak{i}}^{\mathfrak{q}}\right)^{\hat{\omega}_{\mathfrak{i}}} \leq 1-\left(\left(1-\max \left(\mathfrak{K}_{\mathfrak{i}}\right)^{\mathfrak{q}}\right)\right) \\
& \Rightarrow \sqrt[\mathfrak{q}]{1-\left(\left(1-\min \left(\mathfrak{K}_{\mathfrak{i}}\right)^{\mathfrak{q}}\right)\right)} \leq \sqrt[\mathfrak{q}]{\widetilde{\Pi}_{\mathfrak{i}=1}^{\mathfrak{n}}\left(1-\mathfrak{K}_{\mathfrak{i}}^{\mathfrak{q}}\right)^{\omega_{\mathfrak{i}}}} \leq \sqrt[\mathfrak{q}]{1-\left(\left(1-\max \left(\mathfrak{K}_{\mathfrak{i}}\right)^{\mathfrak{q}}\right)\right)} \\
& \Rightarrow \min \left(\mathfrak{K}_{\mathfrak{i}}\right) \leq \sqrt[\mathfrak{q}]{\widetilde{\Pi}_{\mathfrak{i}=1}^{\mathfrak{n}}\left(1-\mathfrak{K}_{\mathfrak{i}}^{\mathfrak{q}}\right)^{\hat{\omega}_{\mathfrak{i}}}} \leq \max \left(\mathfrak{K}_{\mathfrak{i}}\right)
\end{aligned}
$$

As we know, $0 \leq \mathfrak{K}_{\mathfrak{g}}$

leq1, we can write

$\mathfrak{K}_{\mathfrak{g}} \cdot \min \left(\mathfrak{K}_{\mathfrak{i}}\right) \leq \mathfrak{K}_{\mathfrak{g}} \cdot \sqrt[\mathfrak{q}]{\widetilde{\prod}_{\mathfrak{i}=1}^{\mathfrak{n}}\left(1-\mathfrak{K}_{\mathfrak{i}}^{\mathfrak{q}}\right)^{\hat{\omega}_{\mathfrak{i}}}} \leq \mathfrak{K}_{\mathfrak{\mathfrak { g }}} \cdot \max \left(\mathfrak{K}_{\mathfrak{i}}\right)$

$\mathfrak{K}_{\mathfrak{\mathfrak { g }} \otimes \widetilde{\Xi}_{\mathfrak{i}}}^{\min } \leq \mathfrak{K}_{\breve{\mathfrak{g}}} \cdot \sqrt{\widetilde{\prod}_{\mathfrak{i}=1}^{\mathfrak{n}}\left(1-\mathfrak{K}_{\mathfrak{i}}^{\mathfrak{q}}\right)^{\hat{\omega}_{\mathfrak{i}}}} \leq \mathfrak{K}_{\mathfrak{\mathfrak { g }} \otimes \widetilde{\Xi}_{\mathfrak{i}}}^{\max }$

Furthermore, $\min \left(\breve{\Theta}_{\mathfrak{i}}\right) \leq \breve{\Theta}_{\mathfrak{i}} \leq \max \left(\breve{\Theta}_{\mathfrak{i}}\right) \Longleftrightarrow\left(\min \left(\breve{\Theta}_{\mathfrak{i}}\right)\right)^{\mathfrak{q}} \leq \widetilde{\Pi}_{\mathfrak{i}=1}^{\mathfrak{n}}\left(\breve{\Theta}_{\mathfrak{i}}^{\hat{\omega}_{\mathfrak{i}}}\right)^{\mathfrak{q}} \leq\left(\max \left(\breve{\Theta}_{\mathfrak{i}}\right)\right)^{\mathfrak{q}}$. In addition, for $0 \leq \breve{\Theta}_{\mathfrak{g}} \leq 1$, we can write

$$
\begin{aligned}
& \Longrightarrow\left(1-\breve{\Theta}_{\breve{\mathfrak{g}}}^{\mathfrak{q}}\right)\left(\min \left(\breve{\Theta}_{\mathfrak{i}}\right)\right)^{\mathfrak{q}} \leq\left(1-\breve{\Theta}_{\breve{\mathfrak{g}}}^{\mathfrak{q}}\right) \widetilde{\prod}_{\mathfrak{i}=1}^{\mathfrak{n}}\left(\breve{\Theta}_{\mathfrak{i}}^{\hat{\omega}_{\mathfrak{i}}}\right)^{\mathfrak{q}} \leq\left(1-\breve{\Theta}_{\mathfrak{g}}^{\mathfrak{q}}\right)\left(\max \left(\breve{\Theta}_{\mathfrak{i}}\right)\right)^{\mathfrak{q}} \\
& \Longrightarrow \breve{\Theta}_{\mathfrak{\mathfrak { g }}}^{\mathfrak{q}}+\left(1-\breve{\Theta}_{\mathfrak{\mathfrak { g }}}^{\mathfrak{q}}\right)\left(\min \left(\breve{\Theta}_{\mathfrak{i}}\right)\right)^{\mathfrak{q}} \leq \breve{\Theta}_{\breve{\mathfrak{g}}}^{\mathfrak{q}}+\left(1-\breve{\Theta}_{\breve{\mathfrak{g}}}^{\mathfrak{q}}\right) \widetilde{\Pi}_{\mathfrak{i}=1}^{\mathfrak{n}}\left(\breve{\Theta}_{\mathfrak{i}}^{\hat{\omega}_{\mathfrak{i}}}\right)^{\mathfrak{q}} \leq \breve{\Theta}_{\mathfrak{\mathfrak { g }}}^{\mathfrak{q}}+\left(1-\breve{\Theta}_{\mathfrak{\mathfrak { g }}}^{\mathfrak{q}}\right)\left(\max \left(\breve{\Theta}_{\mathfrak{i}}\right)\right)^{\mathfrak{q}} \\
& \Longrightarrow \sqrt[\mathfrak{q}]{\breve{\Theta}_{\mathfrak{g}}^{\mathfrak{q}}+\left(1-\breve{\Theta}_{\breve{\mathfrak{g}}}^{\mathfrak{q}}\right)\left(\min \left(\breve{\Theta}_{\mathfrak{i}}\right)\right)^{\mathfrak{q}}} \leq \sqrt[\mathfrak{q}]{\breve{\Theta}_{\mathfrak{\mathfrak { g }}}^{\mathfrak{q}}+\left(1-\breve{\Theta}_{\mathfrak{\mathfrak { g }}}^{\mathfrak{q}}\right) \widetilde{\prod}_{\mathfrak{i}=1}^{\mathfrak{n}}\left(\breve{\Theta}_{\mathfrak{i}}^{\hat{\omega}_{\mathfrak{i}}}\right)^{\mathfrak{q}}} \leq \sqrt[\mathfrak{q}]{\breve{\Theta}_{\mathfrak{\mathfrak { g }}}^{\mathfrak{q}}+\left(1-\breve{\Theta}_{\mathfrak{g}}^{\mathfrak{q}}\right)\left(\max \left(\breve{\Theta}_{\mathfrak{i}}\right)\right)^{\mathfrak{q}}} \\
& \Longrightarrow \breve{\Theta}_{\breve{\mathfrak{g}} \otimes \widetilde{\Xi}_{\mathfrak{i}}}^{\max } \leq \sqrt[\mathfrak{q}]{\breve{\Theta}_{\mathfrak{\mathfrak { g }}}^{\mathfrak{q}}+\left(1-\breve{\Theta}_{\breve{\mathfrak{g}}}^{\mathfrak{q}}\right) \widetilde{\Pi}_{\mathfrak{i}=1}^{\mathfrak{n}}\left(\breve{\Theta}_{\mathfrak{i}}^{\hat{\omega}_{\mathfrak{i}}}\right)^{\mathfrak{q}}} \leq \breve{\Theta}_{\breve{\mathfrak{g}} \otimes \widetilde{\Xi}_{\mathfrak{i}}}^{\min }
\end{aligned}
$$


GQROFWG $\left(\left(\widetilde{\Xi}_{1}, \widetilde{\Xi}_{2}, \ldots, \widetilde{\Xi}_{\mathfrak{n}}\right), \breve{\mathfrak{g}}\right)=\widetilde{\Xi}=\left(\breve{\Theta}_{\mathfrak{\mathfrak { g }} \otimes} \otimes \widetilde{\Xi}_{\mathfrak{i}}, \mathfrak{K}_{\mathfrak{\mathfrak { g }} \otimes} \otimes \widetilde{\Xi}_{\mathfrak{i}}\right)$, then we have $\mathfrak{K}_{\mathfrak{\mathfrak { g }} \otimes}^{\min } \widetilde{\Xi}_{\mathfrak{i}} \leq \mathfrak{K}_{\mathfrak{\mathfrak { g }} \otimes \widetilde{\Xi}_{\mathfrak{i}}} \leq \mathfrak{K}_{\mathfrak{\mathfrak { g }} \otimes}^{\max } \widetilde{\Xi}_{\mathfrak{i}}$ and $\breve{\Theta}_{\mathfrak{\mathfrak { g }} \otimes}^{\min } \widetilde{\Xi}_{\mathfrak{i}} \leq \breve{\Theta}_{\breve{\mathfrak{g}} \otimes \widetilde{\Xi}_{\mathfrak{i}}} \leq \breve{\Theta}_{\breve{\mathfrak{g}} \otimes \widetilde{\Xi}_{\mathfrak{i}}}^{\max }$. Thus, by definition of score function, we get

$$
\widetilde{\Xi}_{\mathfrak{i}}^{-} \leq \operatorname{GQROFWG}\left(\left(\widetilde{\Xi}_{1}, \widetilde{\Xi}_{2}, \ldots, \widetilde{\Xi}_{\mathfrak{n}}\right), \check{\mathfrak{g}}\right) \leq \widetilde{\Xi}_{\mathfrak{i}}^{+}
$$

3. It can be easily done by the above proof.

4. It follows trivially from definition.

Proposition 15. If the first priority of a another decision expert to the assessed object is considered to be $\breve{\mathfrak{g}}=(0,1)$, then the GQROFWG-operator minimizes in the the $\mathfrak{\mathfrak { q }}$-ROFWG-operator.

Proof. If we take $\breve{g}=(0,1)$ as given then by Theorem 3.2, we have

$$
\begin{aligned}
& \operatorname{GQROFWG~}\left(\left(\widetilde{\Xi}_{1}, \widetilde{\Xi}_{2}, \ldots, \widetilde{\Xi}_{\mathfrak{n}}\right), \check{\mathfrak{g}}\right)=\left(\sqrt[\mathfrak{q}]{\left(\breve{\Theta}_{\mathfrak{\mathfrak { g }}}\right)^{\mathfrak{q}}+\left(1-\left(\breve{\Theta}_{\mathfrak{\mathfrak { g }}}\right)^{\mathfrak{q}}\right) \widetilde{\prod}_{\mathfrak{i}=1}^{\mathfrak{n}}\left(\check{\Theta}_{\mathfrak{i}}^{\hat{\omega}_{\mathfrak{i}}}\right)^{\mathfrak{q}}}, \mathfrak{\mathfrak { K } _ { \mathfrak { g } }} \cdot \sqrt[\mathfrak{q}]{1-\widetilde{\prod}_{i=1}^{\mathfrak{n}}\left(1-\left(\mathfrak{K}_{\mathfrak{i}}\right)^{\mathfrak{q}}\right)^{\hat{\omega}_{\mathfrak{i}}}}\right) \\
& =\left(\sqrt[\mathfrak{q}]{\widetilde{\prod}_{\mathfrak{i}=1}^{\mathfrak{n}}\left(\breve{\Theta}_{i}^{\hat{\omega}_{\mathfrak{i}}}\right)^{\mathfrak{q}}}, \sqrt[\mathfrak{q}]{1-\widetilde{\prod}_{i=1}^{\mathfrak{n}}\left(1-\left(\mathfrak{K}_{\mathfrak{i}}\right)^{\mathfrak{q}}\right)^{\hat{\omega}_{\mathfrak{i}}}}\right) \\
& =\left(\widetilde{\prod}_{\mathfrak{i}=1}^{\mathfrak{n}}\left(\breve{\Theta}_{i}^{\hat{\omega}_{i}}\right)^{\mathfrak{q}}, \sqrt[\mathfrak{q}]{1-\widetilde{\prod}_{i=1}^{\mathfrak{n}}\left(1-\left(\mathfrak{K}_{i}\right)^{\mathfrak{q}}\right)^{\hat{\omega}_{i}}}\right) \\
& =\mathfrak{q}-\operatorname{ROFWG}\left(\widetilde{\Xi}_{1}, \widetilde{\Xi}_{2}, \ldots, \widetilde{\Xi}_{\mathfrak{n}}\right) \text {. }
\end{aligned}
$$

Proposition 16. If the first priority of another decision maker to the assessed object is considered to be $\breve{\mathfrak{g}}=(1,0)$, then the GQROFWG-operator provides the value $(1,0)$.

Proof. If we take $\breve{g}=(1,0)$ as given then by Theorem 3.2, we have

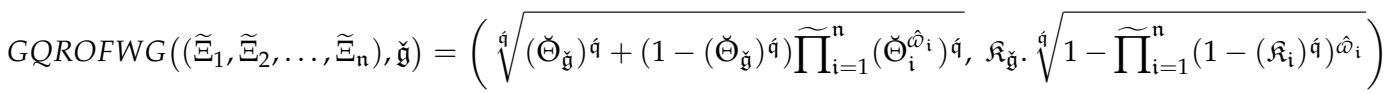

$$
\begin{aligned}
& =\left(\sqrt[\mathfrak{q}]{1+(1-1) \widetilde{\prod}_{\mathfrak{i}=1}^{\mathfrak{n}}\left(\breve{\Theta}_{i}^{\hat{\omega}_{i}}\right)^{\mathfrak{q}}}, 0\right) \\
& =(1,0) \text {. }
\end{aligned}
$$

\subsection{The Generalized q́-ROF Ordered Weighted Geometric Operator}

Definition 17. Let $\check{\mathfrak{g}}=\left(\breve{\Theta}_{\breve{\mathfrak{g}}}, \mathfrak{K}_{\breve{\mathfrak{g}}}\right)$ be a GP for the $\mathfrak{\mathfrak { q }}-R O F N s \widetilde{\Xi}_{\mathfrak{i}}=\left(\breve{\Theta}_{\mathfrak{i}}, \mathfrak{K}_{\mathfrak{i}}\right)(\mathfrak{i}=1,2, \ldots, \mathfrak{n})$, then the GQROFOWG-operator is characterized as,

$$
\operatorname{GQROFWG}\left(\left(\widetilde{\Xi}_{1}, \widetilde{\Xi}_{2}, \ldots, \widetilde{\Xi}_{\mathfrak{n}}, \check{\mathfrak{g}}\right)\right)=\check{\mathfrak{g}} \otimes \mathfrak{q}-\operatorname{ROFOWG}\left(\widetilde{\Xi}_{1}, \widetilde{\Xi}_{2}, \ldots, \widetilde{\Xi}_{\mathfrak{n}}\right)
$$

Theorem 18. Let $\widetilde{\Xi}_{\mathfrak{i}}=\left(\breve{\Theta}_{\mathfrak{i}}, \mathfrak{K}_{\mathfrak{i}}\right)(\mathfrak{i}=1,2, \ldots, \mathfrak{n})$ be a set of $\mathfrak{\mathfrak { q }}$-ROFNs and $\hat{\omega}=\left(\hat{\omega}_{1}, \hat{\omega}_{2}, \ldots, \hat{\omega}_{\mathfrak{n}}\right)^{T}$ is the weight vector of $\widetilde{\Xi}_{\mathfrak{i}}$ such that $\hat{\omega}_{\mathfrak{i}} \in[0,1]$ and $\sum_{\mathfrak{i}=1}^{\mathfrak{n}}$. GP is $\check{\mathfrak{g}}=\left(\breve{\Theta}_{\mathfrak{\mathfrak { g }}}, \mathfrak{K}_{\mathfrak{\mathfrak { g }}}\right)$, then the GQROFOWG-operator is defined as

$$
\begin{aligned}
\operatorname{GQROFWG}\left(\left(\widetilde{\Xi}_{1}, \widetilde{\Xi}_{2}, \ldots, \widetilde{\Xi}_{\mathfrak{n}}\right), \check{\mathfrak{g}}\right) & =\mathfrak{g} \otimes \mathfrak{q}-\operatorname{ROFOWG}\left(\widetilde{\Xi}_{1}, \widetilde{\Xi}_{2}, \ldots, \widetilde{\Xi}_{\mathfrak{n}}\right) \\
& =\left(\sqrt[\mathfrak{q}]{\left(\breve{\Theta}_{\mathfrak{\mathfrak { g }}}\right)^{\mathfrak{q}}+\left(1-\left(\breve{\Theta}_{\mathfrak{\mathfrak { g }}}\right)^{\mathfrak{q}}\right) \widetilde{\prod}_{\mathfrak{i}=1}^{\mathfrak{n}}\left(\breve{\Theta}_{\sigma(\mathfrak{i})}^{\mathfrak{\omega}_{\mathfrak{i}}}\right)}, \mathfrak{q}, \mathfrak{K}_{\mathfrak{\mathfrak { g }}} \cdot \sqrt[\mathfrak{q}]{1-\widetilde{\prod}_{\mathfrak{i}=1}^{\mathfrak{n}}\left(1-\left(\mathfrak{K}_{\sigma(\mathfrak{i})}\right)^{\mathfrak{q}}\right)^{\hat{\omega}_{\mathfrak{i}}}}\right)
\end{aligned}
$$


$(\sigma(1), \sigma(2), \ldots, \sigma(\mathfrak{n}))$ is a permutation of $(1,2, \ldots, \mathfrak{n})$, such that $\widetilde{\Xi}_{\sigma(\mathfrak{i}-1)} \geq \widetilde{\Xi}_{\sigma(\mathfrak{i})}$ for any $\mathfrak{i}$.

Proof. The proof can be done as Theorem 3.2.

Example 19. Let $\check{\mathfrak{g}}=(0.5,0.7)$ be the GP of four $\mathfrak{\mathfrak { q }}-$ ROPFNs. $\widetilde{\Xi}_{1}=(0.23,0.67), \widetilde{\Xi}_{2}=(0.42,0.77)$, $\widetilde{\Xi}_{3}=(0.78,0.55)$ and $\widetilde{\Xi}_{4}=(0.41,0.84)$ with a weight vector $\hat{\omega}=(0.1,0.2,0.3,0.4)$, here $\mathfrak{q}=3$, then first we find score functions of all $\widetilde{\Xi}_{\mathfrak{i}}$.

$$
\begin{aligned}
\widetilde{\tau}\left(\widetilde{\Xi}_{1}\right) & =-0.2885 \\
\widetilde{\tau}\left(\widetilde{\Xi}_{2}\right) & =-0.3824 \\
\widetilde{\tau}\left(\widetilde{\Xi}_{3}\right) & =0.3081 \\
\widetilde{\tau}\left(\widetilde{\Xi}_{4}\right) & =-0.5237
\end{aligned}
$$

On the behalf of score functions, $\widetilde{\Xi}_{\sigma(1)}=\widetilde{\Xi}_{3}, \widetilde{\Xi}_{\sigma(2)}=\widetilde{\Xi}_{1}, \widetilde{\Xi}_{\sigma(3)}=\widetilde{\Xi}_{2}$, and $\widetilde{\Xi}_{\sigma(4)}=\widetilde{\Xi}_{4}$

$$
\sqrt[\mathfrak{q}]{\left(\breve{\Theta}_{\breve{\mathfrak{g}}}\right) \mathfrak{q}+\left(1-\left(\breve{\Theta}_{\breve{\mathfrak{g}}}\right) \mathfrak{\mathfrak { q }}\right) \widetilde{\prod}_{\mathfrak{i}=1}^{\mathfrak{n}}\left(\breve{\Theta}_{\sigma(\mathfrak{i})}^{\hat{\omega}_{\mathfrak{i}}}\right) \mathfrak{\mathfrak { q }}}=0.5623
$$

In addition,

$$
\mathfrak{K}_{\mathfrak{\mathfrak { g }}} \cdot \sqrt[\mathfrak{q}]{1-\widetilde{\prod}_{\mathfrak{i}=1}^{\mathfrak{n}}\left(1-\left(\mathfrak{K}_{\sigma(\mathfrak{i})}\right)^{\mathfrak{q}}\right)^{\hat{\omega}_{\mathfrak{i}}}}=0.5436
$$

By Theorem 3.9, we have

$$
\begin{aligned}
\operatorname{GQROFOWG}\left(\left(\widetilde{\Xi}_{1}, \widetilde{\Xi}_{2}, \widetilde{\Xi}_{3}, \widetilde{\Xi}_{4}\right), \check{\mathfrak{g}}\right) & =\check{\mathfrak{g}} \otimes \mathfrak{q}-R O F O W G\left(\widetilde{\Xi}_{1}, \widetilde{\Xi}_{2}, \ldots, \widetilde{\Xi}_{\mathfrak{n}}\right) \\
& =\left(\sqrt[\mathfrak{q}]{\left(\breve{\Theta}_{\breve{\mathfrak{g}}}\right)^{\mathfrak{q}}+\left(1-\left(\breve{\Theta}_{\breve{\mathfrak{g}}}\right)^{\mathfrak{q}}\right) \widetilde{\prod}_{\mathfrak{i}=1}^{\mathfrak{k}}\left(\breve{\Theta}_{\mathfrak{i}}^{\hat{\omega}_{\mathfrak{i}}}\right) \mathfrak{q},} \mathfrak{K}_{\mathfrak{\mathfrak { g }}} \cdot \sqrt[\mathfrak{q}]{1-\widetilde{\prod}_{\mathfrak{i}=1}^{\mathfrak{k}}\left(1-\left(\mathfrak{K}_{\mathfrak{i}}\right) \mathfrak{q}\right) \hat{\mathfrak{\omega}}_{\mathfrak{i}}}\right) \\
& =(0.5623,0.5436)
\end{aligned}
$$

Proposition 20. Let $\widetilde{\Xi}_{\mathfrak{i}}=\left(\breve{\Theta}_{\mathfrak{i}}, \mathfrak{K}_{\mathfrak{i}}\right)(\mathfrak{i}=1,2, \ldots \mathfrak{n})$ be a set of $\mathfrak{\mathfrak { q }}-R O F N s$ and $\hat{\omega}=\left(\hat{\omega}_{1}, \hat{\omega}_{2}, \ldots, \hat{\omega}_{\mathfrak{n}}\right)^{T}$ is the weight vector of $\widetilde{\Xi}_{\mathfrak{i}}$ such that $\hat{\omega}_{\mathfrak{i}} \in[0,1]$ and $\sum_{\mathfrak{i}=1}^{\mathfrak{n}}$. Generalized parameter is $\breve{\mathfrak{g}}=\left(\breve{\Theta}_{\mathfrak{g}}, \mathfrak{K}_{\mathfrak{g}}\right)$, the GQROFOWG-operator has the following properties:

1. (Idempotency) If $\widetilde{\Xi}_{\mathfrak{i}}=\widetilde{\Xi}(\forall \mathfrak{i}=1,2, \ldots, \mathfrak{n})$, then

$$
\operatorname{GQROFOWG}\left(\left(\widetilde{\Xi}_{1}, \widetilde{\Xi}_{2}, \ldots, \widetilde{\Xi}_{\mathfrak{n}}\right), \check{\mathfrak{g}}\right)=\check{\mathfrak{g}} \otimes \widetilde{\Xi}
$$

2. (Boundary condition) If $\widetilde{\Xi}_{\mathfrak{i}}^{-}=\left(\breve{\Theta}_{\breve{\mathfrak{g}} \otimes \widetilde{\Xi}_{\mathfrak{i}}}^{\min }, \mathfrak{K}_{\breve{\mathfrak{g}} \otimes}^{\max } \widetilde{\Xi}_{\mathfrak{i}}\right)$ and $\widetilde{\Xi}_{\mathfrak{i}}^{+}=\left(\breve{\Theta}_{\breve{\mathfrak{g}} \otimes}^{\max }, \widetilde{\mathfrak{\Xi}}_{\mathfrak{i}}, \mathfrak{K}_{\mathfrak{\mathfrak { g }} \otimes}^{\min } \widetilde{\Xi}_{\mathfrak{i}}\right)$, then for every $\hat{\omega}_{\mathfrak{i}}$,

$$
\widetilde{\Xi}_{\mathfrak{i}}^{-} \leq \operatorname{GQROFOWG}\left(\left(\widetilde{\Xi}_{1}, \widetilde{\Xi}_{2}, \ldots, \widetilde{\Xi}_{\mathfrak{n}}\right), \mathfrak{g}\right) \leq \widetilde{\Xi}_{\mathfrak{i}}^{+}
$$

3. (Monotonicity) Let $\widetilde{\Xi}_{\mathfrak{i}}^{\star}=\left(\breve{\Theta}_{\mathfrak{i}}^{\star}, \mathfrak{K}_{\mathfrak{i}}^{\star}\right)(\mathfrak{i}=1,2, \ldots, \mathfrak{n})$ be a set of $\mathfrak{q}-R O F N s$ such that $\mathfrak{K}_{\mathfrak{i}}^{\star} \leq \mathfrak{K}_{\mathfrak{i}}$ and $\breve{\Theta}_{\mathfrak{i}} \leq \breve{\Theta}_{\mathfrak{i}}^{\star}$ for all $\mathfrak{i}$, then for every $\hat{\omega}_{i}$,

$$
\text { GQROFOWG }\left(\left(\widetilde{\Xi}_{1}, \widetilde{\Xi}_{2}, \ldots, \widetilde{\Xi}_{\mathfrak{n}}\right), \check{\mathfrak{g}}\right) \leq \text { GQROFOWG }\left(\left(\widetilde{\Xi}_{1}^{\star}, \widetilde{\Xi}_{2}^{\star}, \ldots, \widetilde{\Xi}_{\mathfrak{n}}^{\star}\right), \check{\mathfrak{g}}\right)
$$

4. (Commutativity) Let $\widetilde{\Xi}_{\mathfrak{i}}=\left(\breve{\Theta}_{\mathfrak{i}}, \mathfrak{K}_{\mathfrak{i}}\right)(\mathfrak{i}=1,2, \ldots, \mathfrak{n})$ and $\widetilde{\Xi}_{\mathfrak{i}}^{*}=\left(\breve{\Theta}_{\mathfrak{i}}^{*}, \mathfrak{K}_{\mathfrak{i}}{ }^{*}\right)(\mathfrak{i}=1,2, \ldots, \mathfrak{n})$ be a two collection of $\mathfrak{n} \mathfrak{q}-R O F N$ s such that $\widetilde{\Xi}_{\mathfrak{i}}^{*}$ is any permutation of $\widetilde{\Xi}_{\mathfrak{i}}$, then

$$
\operatorname{GQROFOWG}\left(\left(\widetilde{\Xi}_{1}, \widetilde{\Xi}_{2}, \ldots, \widetilde{\Xi}_{\mathfrak{n}}\right), \check{\mathfrak{g}}\right)=\operatorname{GQROFOWG}\left(\left(\widetilde{\Xi}_{1}^{*}, \widetilde{\Xi}_{2}^{*}, \ldots, \widetilde{\Xi}_{\mathfrak{n}}^{*}\right), \mathfrak{g}\right)
$$


5. If the preference of another decision maker to the assessed object is considered to be $\breve{\mathfrak{g}}=(0,1)$, then the GQROFOWG-operator becomes the $\mathfrak{q}$-ROFOWG-operator.

6. If the preference of another decision maker to the assessed object is considered to be $\breve{\mathfrak{g}}=(1,0)$, then the GQROFOWG-operator provides the value $(1,0)$.

Proof. Here we leave proof.

\subsection{The Generalized q́-ROF Hybrid Geometric Operator}

Definition 21. Suppose $\check{\mathfrak{g}}=\left(\breve{\Theta}_{\breve{\mathfrak{g}}}, \mathfrak{K}_{\mathfrak{g}}\right)$ be the generalized parameter for the $\mathfrak{\mathfrak { q }}$-ROFNs $\widetilde{\Xi}_{\mathfrak{i}}=\left(\breve{\Theta}_{\mathfrak{i}}, \mathfrak{K}_{\mathfrak{i}}\right)(\mathfrak{i}=1,2, \ldots, \mathfrak{n})$, then the GQROFHG-operator is determined as,

$$
\operatorname{GQROFHG}\left(\left(\widetilde{\Xi}_{1}, \widetilde{\Xi}_{2}, \ldots, \widetilde{\Xi}_{\mathfrak{n}}, \mathfrak{g}\right)\right)=\check{\mathfrak{g}} \otimes \mathfrak{q}-R O F H G\left(\widetilde{\Xi}_{1}, \widetilde{\Xi}_{2}, \ldots, \widetilde{\Xi}_{\mathfrak{n}}\right)
$$

Theorem 22. Let $\widetilde{\Xi}_{\mathfrak{i}}=\left(\breve{\Theta}_{\mathfrak{i}}, \mathfrak{K}_{\mathfrak{i}}\right)(\mathfrak{i}=1,2, \ldots, \mathfrak{n})$ be a set of $\mathfrak{\mathfrak { q }}-R O F N s$ and $\hat{\boldsymbol{\omega}}=\left(\hat{\omega}_{1}, \hat{\omega}_{2}, \ldots, \hat{\omega}_{\mathfrak{n}}\right)^{T}$ be a weight vector of $\widetilde{\Xi}_{\mathfrak{i}}$ such that $\hat{\omega}_{\mathfrak{i}} \in[0,1]$ and $\sum_{\mathfrak{i}=1}^{\mathfrak{n}} \hat{\omega}_{\mathfrak{i}}=1$. The GP is $\breve{\mathfrak{g}}=\left(\breve{\Theta}_{\breve{\mathfrak{g}}}, \mathfrak{K}_{\mathfrak{\mathfrak { g }}}\right)$ and the standard vector is $\xi=\left(\xi_{1}, \xi_{2}, \ldots, \xi_{\mathfrak{n}}\right)^{T}$ such that $\xi_{\mathfrak{i}} \in[0,1]$ and $\sum_{\mathfrak{i}=1}^{\mathfrak{n}} \xi_{\mathfrak{i}}=1$. The GQROFHG-operator is determined as,

$$
\begin{aligned}
& \operatorname{GQROFHG}\left(\left(\widetilde{\Xi}_{1}, \widetilde{\Xi}_{2}, \ldots, \widetilde{\Xi}_{\mathfrak{n}}\right), \check{\mathfrak{g}}\right)=\check{\mathfrak{g}} \otimes \mathfrak{q}-R O F H G\left(\widetilde{\Xi}_{1}, \widetilde{\Xi}_{2}, \ldots, \widetilde{\Xi}_{\mathfrak{n}}\right) \\
& =\left(\sqrt[\mathfrak{q}]{\left(\breve{\Theta}_{\mathfrak{\mathfrak { g }}}\right)^{\mathfrak{q}}+\left(1-\left(\breve{\Theta}_{\mathfrak{\mathfrak { g }}}\right) \mathfrak{q}\right) \widetilde{\prod}_{\mathfrak{i}=1}^{\mathfrak{n}}\left(\breve{\Theta}_{\sigma(\mathfrak{i})}^{\hat{\omega}_{\mathfrak{i}}}\right) \mathfrak{q},} \cdot \sqrt[\mathfrak{K}_{\mathfrak{\mathfrak { g }}}]{\cdot \mathfrak{q}} \sqrt{1-\widetilde{\prod}_{\mathfrak{i}=1}^{\mathfrak{n}}\left(1-\left(\breve{\mathfrak{K}}_{\sigma(\mathfrak{i})}\right) \mathfrak{q}\right) \hat{\omega}_{\mathfrak{i}}}\right)
\end{aligned}
$$

here $\breve{\Xi}_{\mathfrak{i}}=\mathfrak{n} \xi_{\mathfrak{i}} \widetilde{\Xi}_{\mathfrak{i}}, \mathfrak{n}$ is the number of $\mathfrak{\mathfrak { q }}-R O F N$ s, $\boldsymbol{\xi}_{\mathfrak{i}}$ is a standard weight vector of $\widetilde{\Xi}_{\mathfrak{i}}$, and $(\sigma(1), \sigma(2), \ldots, \sigma(\mathfrak{n}))$ is a permutation of $(1,2, \ldots, \mathfrak{n})$, such that $\breve{\Xi}_{\sigma(\mathfrak{i}-1)} \geq \breve{\Xi}_{\sigma(\mathfrak{i})}$ for any $\mathfrak{i}$.

Proof. The proof can be done same as Theorem 3.2.

Example 23. Let $\mathfrak{\mathfrak { g }}=(0.5,0.7)$ be the GP of four $\mathfrak{q}$-rung orthopair fuzzy numbers. $\widetilde{\Xi}_{1}=(0.23,0.67)$, $\widetilde{\Xi}_{2}=(0.42,0.77), \widetilde{\Xi}_{3}=(0.78,0.55)$, and $\widetilde{\Xi}_{3}=(0.41,0.84)$ with a weight vector $\mathcal{\omega}=(0.1,0.2,0.3,0.4)$, here $\mathfrak{q}=4$. Standard weight vector will be $\xi_{\mathfrak{i}}=(0.4,0.3,0.2,0.1)$. First we find $\widetilde{\Xi}_{\mathfrak{i}}=\mathfrak{n} \widetilde{\xi}_{\mathfrak{i}} \widetilde{\Xi}_{\mathfrak{i}}$ for each $\widetilde{\Xi}_{\mathfrak{i}}$, then we find score functions of each $\breve{\Xi}_{\mathfrak{i}}$.

$$
\begin{aligned}
& \breve{\Xi}_{1}=(0.258622,0.526889) \\
& \breve{\Xi}_{2}=(0.439241,0.730783) \\
& \breve{\Xi}_{3}=(0.745657,0.619855) \\
& \breve{\Xi}_{4}=(0.326760,0.932635)
\end{aligned}
$$

The score functions will be,

$$
\begin{gathered}
\widetilde{\neg}\left(\widetilde{\Xi}_{1}\right)=-0.072594 \\
\widetilde{\neg}\left(\widetilde{\Xi}_{2}\right)=-0.247979 \\
\widetilde{\neg}\left(\breve{\Xi}_{3}\right)=0.161515 \\
\widetilde{\neg}\left(\widetilde{\Xi}_{3}\right)=-0.745165
\end{gathered}
$$

On the behalf of score functions, $\widetilde{\Xi}_{\sigma(1)}=\breve{\Xi}_{3}, \breve{\Xi}_{\sigma(2)}=\breve{\Xi}_{1}, \widetilde{\Xi}_{\sigma(3)}=\breve{\Xi}_{2}$, and $\widetilde{\Xi}_{\sigma(4)}=\breve{\Xi}_{4}$

$$
\sqrt[\mathfrak{q}]{\left(\breve{\Theta}_{\breve{\mathfrak{g}}}\right)^{\mathfrak{q}}+\left(1-\left(\breve{\Theta}_{\breve{\mathfrak{g}}}\right)^{\mathfrak{q}}\right) \widetilde{\prod}_{\mathfrak{i}=1}^{\mathfrak{n}}\left(\breve{\Theta}_{\sigma(\mathfrak{i})}^{\hat{\omega}_{\mathfrak{i}}}\right) \mathfrak{q}}=0.531970
$$


In addition,

$$
\mathfrak{K}_{\mathfrak{\mathfrak { g }}} \cdot \sqrt[\mathfrak{q}]{1-\widetilde{\prod}_{\mathfrak{i}=1}^{\mathfrak{n}}\left(1-\left(\breve{\mathfrak{K}}_{\sigma(\mathfrak{i})}\right)^{\mathfrak{q}}\right)^{\hat{\omega}_{\mathfrak{i}}}}=0.589324
$$

By Theorem 3.13, we have

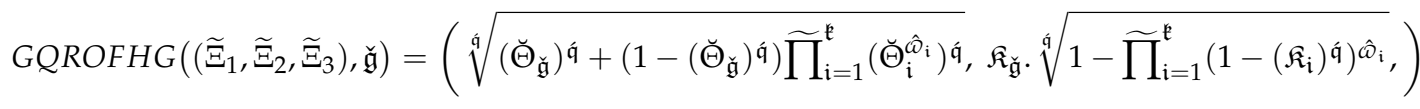

$$
\begin{aligned}
& =(0.531970,0.589324)
\end{aligned}
$$

The following observation are derived from definition of GQROFHG-operator:

1. If the preference of another decision maker to the assessed object is considered to be $\breve{\mathfrak{g}}=(0,1)$, then the GQROFHG-operator becomes the q́-ROFHG-operator.

2. If the preference of another decision maker to the assessed object is considered to be $\breve{\mathfrak{g}}=(1,0)$, then the GQROFHG-operator provides the value $(1,0)$.

3. If $\xi=\left(\frac{1}{\mathfrak{n}}, \frac{1}{\mathfrak{n}}, \ldots, \frac{1}{\mathfrak{n}}\right)^{T}$, then the GQROFHG-operator reduces to GQROFWG-operator.

4. If $\hat{\omega}=\left(\frac{1}{\mathfrak{n}}, \frac{1}{\mathfrak{n}}, \ldots, \frac{1}{\mathfrak{n}}\right)^{T}$, then the GQROFHG-operator reduces to GQROFOWG-operator.

\section{5. q́-ROF Geometric Aggregation Operator Based On Group-Generalized Parameter}

The presented section is dedicated to extending collaborators above geometric aggregation operators by taking the conceptions of different specialists/decision experts on the preliminary information to better integrate different preferences of decision makers. This can be obtained by providing a group-generalized q́-rung orthopair fuzzy weighted geometric(GGQROFWG-operator), group-generalized q́ -rung orthopair fuzzy ordered weighted geometric(GGQROFOWG-operator) and group-generalized q́-rung orthopair fuzzy hybrid geometric(GGQROFHG-operator).

\subsection{Group-Generalized q́-ROF Weighted Geometric Operator}

Definition 24. Suppose there are $\mathfrak{q}$ specialists/decision experts to verify the $\mathfrak{q}$-ROF information. Let $\mathfrak{g}_{z}=\left(\breve{\Theta}_{\mathfrak{\mathfrak { g }}_{z}}, \mathfrak{K}_{\mathfrak{\mathfrak { g }}_{z}}\right)$ be the specialists/decision experts for the $\mathfrak{\mathfrak { q }}-R O F N s \widetilde{\Xi}_{\mathfrak{i}}=\left(\breve{\Theta}_{\mathfrak{i}}, \mathfrak{K}_{\mathfrak{i}}\right)(\mathfrak{i}=1,2, \ldots, \mathfrak{n})$, then GGQROFWG-operator is determined as,

$$
\operatorname{GGQROFWG}\left(\left(\widetilde{\Xi}_{1}, \widetilde{\Xi}_{2}, \ldots, \widetilde{\Xi}_{\mathfrak{n}}\right),\left(\check{\mathfrak{g}}_{1}, \check{\mathfrak{g}}_{2}, \ldots, \check{\mathfrak{g}}_{\mathfrak{q}}\right)\right)=\mathfrak{\mathfrak { q }}-\operatorname{ROFWG}\left(\check{\mathfrak{g}}_{1}, \check{\mathfrak{g}}_{2}, \ldots, \check{\mathfrak{g}}_{\mathfrak{q}}\right) \otimes \mathfrak{q}-R O F W G\left(\widetilde{\Xi}_{1}, \widetilde{\Xi}_{2}, \ldots, \widetilde{\Xi}_{\mathfrak{n}}\right)
$$

Theorem 25. Let there be $\mathfrak{q}$ specialists/decision experts to verify the $\mathfrak{\mathfrak { q }}$-ROF information. Let $\check{\mathfrak{g}}_{z}=\left(\breve{\Theta}_{\check{\mathfrak{g}}_{z}}, \mathfrak{K}_{\check{\mathfrak{g}}_{z}}\right)$ $(\mathfrak{i}=1,2, \ldots, \mathfrak{q})$ be the specialists/decision experts for the $\mathfrak{\mathfrak { q }}$-ROFNs $\widetilde{\Xi}_{\mathfrak{i}}=\left(\breve{\Theta}_{\mathfrak{i}}, \mathfrak{K}_{\mathfrak{i}}\right)(\mathfrak{i}=1,2, \ldots, \mathfrak{n})$. $\hat{\omega}^{\prime}=\left(\hat{\omega}_{1}^{\prime}, \hat{\omega}_{2}^{\prime}, \ldots, \hat{\omega}_{\hat{q}}^{\prime}\right)^{T}$ and $\hat{\omega}=\left(\hat{\omega}_{1}, \omega_{2}, \ldots, \hat{\omega}_{\mathfrak{n}}\right)^{T}$ are the weight vectors of specialists/decision experts and $\widetilde{\Xi}_{\mathfrak{i}}$, respectively and $\hat{\omega}_{\mathfrak{i}}^{\prime} \in[0,1], \sum_{\mathfrak{i}=1}^{\mathfrak{q}} \hat{\omega}_{\mathfrak{i}}^{\prime}=1, \hat{\omega}_{\mathfrak{i}} \in[0,1]$, and $\sum_{\mathfrak{i}=1}^{\mathfrak{n}} \hat{\omega}_{\mathfrak{i}}=1$, then the GGQROFWG-operator is determined as,

$$
\begin{aligned}
& \operatorname{GGQROFWG}\left(\left(\widetilde{\Xi}_{1}, \widetilde{\Xi}_{2}, \ldots, \widetilde{\Xi}_{\mathfrak{n}}\right),\left(\mathfrak{g}_{1}, \check{\mathfrak{g}}_{2}, \ldots, \check{\mathfrak{g}}_{\mathfrak{q}}\right)\right)=\mathfrak{q}-\operatorname{ROFWG}\left(\check{\mathfrak{g}}_{1}, \check{\mathfrak{g}}_{2}, \ldots, \check{\mathfrak{g}}_{\mathfrak{q}}\right) \otimes \mathfrak{q}-R O F W G\left(\widetilde{\Xi}_{1}, \widetilde{\Xi}_{2}, \ldots, \widetilde{\Xi}_{\mathfrak{n}}\right) \\
& =\left(\sqrt[\mathfrak{q}]{\widetilde{\prod}_{z=1}^{\mathfrak{q}}\left(\breve{\Theta}_{\breve{\mathfrak{g}}_{z}}^{\hat{\omega}_{z}^{\prime}}\right)^{\mathfrak{q}}+\widetilde{\prod}_{\mathfrak{i}=1}^{\mathfrak{n}}\left(\breve{\Theta}_{\mathfrak{i}}^{\hat{\omega}_{\mathfrak{i}}}\right)^{\mathfrak{q}}-\widetilde{\prod}_{z=1}^{\mathfrak{q}}\left(\breve{\Theta}_{\mathfrak{\mathfrak { q }}_{z}}^{\omega_{z}^{\prime}}\right)^{\mathfrak{q}} \cdot \widetilde{\prod}_{\mathfrak{i}=1}^{\mathfrak{n}}\left(\breve{\Theta}_{i}^{\hat{\omega}_{\mathfrak{i}}}\right)^{\mathfrak{q}}},\right.
\end{aligned}
$$

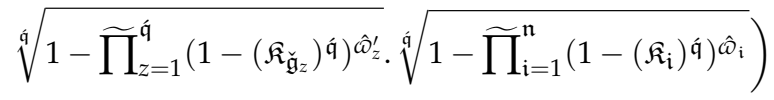

Proof. We will use mathematical induction. 
For $\mathfrak{n}=2$,

$$
\begin{aligned}
& \operatorname{GGQROFWG}\left(\left(\widetilde{\Xi}_{1}, \widetilde{\Xi}_{2}\right),\left(\check{\mathfrak{g}}_{1}, \mathfrak{g}_{2}, \ldots, \check{\mathfrak{g}}_{\mathfrak{q}}\right)\right)=\mathfrak{q}-\operatorname{ROFWG}\left(\check{\mathfrak{g}}_{1}, \check{\mathfrak{g}}_{2}, \ldots, \check{\mathfrak{g}}_{\mathfrak{q}}\right) \otimes \mathfrak{\mathfrak { q }}-\operatorname{ROFWG}\left(\widetilde{\Xi}_{1}, \widetilde{\Xi}_{2}\right) \\
& \left.=\left(\widetilde{\prod}_{k=1}^{\mathfrak{n}} \breve{\Theta}_{\mathfrak{\mathfrak { g }}_{z}}^{\hat{\omega}_{z}^{\prime}}, \sqrt[\mathfrak{q}]{1-\widetilde{\prod}_{z=1}^{\mathfrak{q}}\left(1-\left(\mathfrak{K}_{\mathfrak{g}_{z}}\right)\right.}\right) \hat{\mathfrak{q}}^{\mathfrak{q} \hat{\omega}_{z}^{\prime}}\right) \otimes\left(\widetilde{\Xi}_{1}^{\hat{\omega}_{1}} \otimes \widetilde{\Xi}_{2}^{\hat{\omega}_{2}}\right) \\
& =\left(\widetilde{\prod}_{k=1}^{\mathfrak{n}} \breve{\Theta}_{\mathfrak{\mathfrak { g }}_{z}}^{\hat{\omega}_{z}^{\prime}}, \sqrt[\mathfrak{q}]{1-\widetilde{\prod}_{z=1}^{\mathfrak{q}}\left(1-\left(\mathfrak{K}_{\mathfrak{\mathfrak { g }}_{z}}\right)^{\mathfrak{q}}\right)^{\hat{\omega}_{z}^{\prime}}}\right) \\
& \otimes\left(\breve{\Theta}_{1}^{\hat{\omega}_{1}} \cdot \breve{\Theta}_{2}^{\hat{\omega}_{2}}, \sqrt[\mathfrak{q}]{1-\left(1-\mathfrak{K}_{1}^{\mathfrak{q}}\right)^{\hat{\omega}_{1}} \cdot\left(1-\mathfrak{K}_{2}^{\mathfrak{q}}\right)^{\hat{\omega}_{2}}}\right)
\end{aligned}
$$

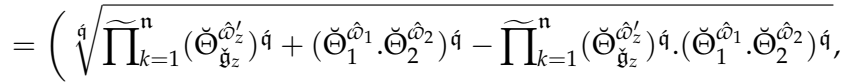

$$
\begin{aligned}
& \left.\sqrt[\mathfrak{q}]{1-\widetilde{\prod}_{z=1}^{\mathfrak{q}}\left(1-\left(\mathfrak{K}_{\check{\mathfrak{g}}_{z}}\right) \mathfrak{q}\right)^{\hat{\omega}_{z}^{\prime}}} \cdot \sqrt[\mathfrak{q}]{1-\left(1-\mathfrak{K}_{1}^{\mathfrak{q}}\right)^{\hat{\omega}_{1}} \cdot\left(1-\mathfrak{K}_{2}^{\mathfrak{q}}\right)^{\hat{\omega}_{2}}}\right) \\
& =\left(\sqrt[\mathfrak{q}]{\widetilde{\prod}_{z=1}^{\mathfrak{q}}\left(\breve{\Theta}_{\mathfrak{\mathfrak { g }}_{z}}^{\hat{\omega}_{z}^{\prime}}\right)^{\mathfrak{q}}+\widetilde{\prod}_{\mathfrak{i}=1}^{2}\left(\breve{\Theta}_{\mathfrak{i}}^{\hat{\omega}_{\mathfrak{i}}}\right)^{\mathfrak{q}}-\widetilde{\prod}_{z=1}^{\mathfrak{q}}\left(\breve{\Theta}_{\mathfrak{\mathfrak { g }}_{z}}^{\prime}\right)^{\prime} \cdot \widetilde{\prod}_{\mathfrak{i}=1}^{2}\left(\breve{\Theta}_{\mathfrak{i}}^{\hat{\omega}_{\mathfrak{i}}}\right)^{\mathfrak{q}}},\right.
\end{aligned}
$$

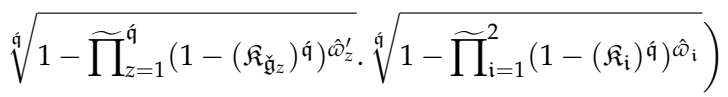

For $\mathfrak{n}=2$, result is satisfied.

Suppose result is true for $\mathfrak{n}=\mathfrak{k}$,

$\operatorname{GGQROFWG}\left(\left(\widetilde{\Xi}_{1}, \widetilde{\Xi}_{2}, \ldots, \widetilde{\Xi}_{k}\right),\left(\check{\mathfrak{g}}_{1}, \check{\mathfrak{g}}_{2}, \ldots, \check{\mathfrak{g}}_{\mathfrak{q}}\right)\right)=\mathfrak{q}-\operatorname{ROFWG}\left(\check{\mathfrak{g}}_{1}, \check{\mathfrak{g}}_{2}, \ldots, \check{\mathfrak{g}}_{\mathfrak{q}}\right) \otimes \mathfrak{q}-\operatorname{ROFWG}\left(\widetilde{\Xi}_{1}, \widetilde{\Xi}_{2}, \ldots, \widetilde{\Xi}_{k}\right)$

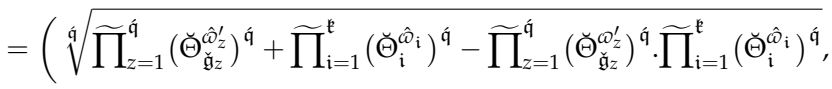

$$
\begin{aligned}
& \sqrt[\mathfrak{q}]{1-\widetilde{\prod}_{z=1}^{\mathfrak{q}}\left(1-\left(\mathfrak{K}_{\mathfrak{\mathfrak { g }}_{z}}\right)^{\mathfrak{q}}\right) \hat{\omega}_{z}} \cdot \sqrt[\mathfrak{q}]{\left.1-\widetilde{\prod}_{\mathfrak{i}=1}^{\mathfrak{k}}\left(1-\left(\mathfrak{K}_{\mathfrak{i}}\right)^{\mathfrak{q}}\right)^{\hat{\boldsymbol{i}}_{\mathfrak{i}}},\right)}
\end{aligned}
$$

For $\mathfrak{n}=\mathfrak{k}+1$, we will prove

$\operatorname{GGQROFWG~}\left(\left(\widetilde{\Xi}_{1}, \widetilde{\Xi}_{2}, \ldots, \widetilde{\Xi}_{k+1}\right),\left(\check{\mathfrak{g}}_{1}, \breve{\mathfrak{g}}_{2}, \ldots, \check{\mathfrak{g}}_{\mathfrak{q}}\right)\right)=\mathfrak{q}-\operatorname{ROFWG}\left(\breve{\mathfrak{g}}_{1}, \breve{\mathfrak{g}}_{2}, \ldots, \check{\mathfrak{g}}_{\mathfrak{q}}\right) \otimes \mathfrak{q}-\operatorname{ROFWG}\left(\widetilde{\Xi}_{1}, \widetilde{\Xi}_{2}, \ldots, \widetilde{\Xi}_{k+1}\right)$

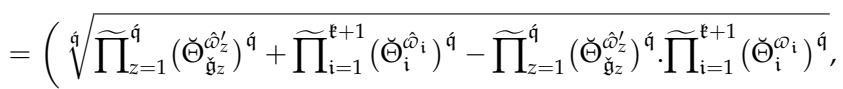

$$
\begin{aligned}
& \left.\sqrt[\mathfrak{q}]{1-\widetilde{\prod}_{z=1}^{\mathfrak{q}}\left(1-\left(\mathfrak{K}_{\mathfrak{g}}\right)^{\mathfrak{q}}\right) \hat{\omega}_{z}^{\prime}} \cdot \sqrt[\mathfrak{q}]{1-\widetilde{\prod}_{\mathfrak{i}=1}^{\mathfrak{k}+1}\left(1-\left(\mathfrak{K}_{\mathfrak{i}}\right)^{\mathfrak{q}}\right)^{\hat{\omega}_{\mathfrak{i}}}}\right)
\end{aligned}
$$

Therefore, the result satisfied for $\mathfrak{n}=\mathfrak{k}+1$, under more than one specialist's/decision expert's preference.

Example 26. Let $\check{\mathfrak{g}}_{z}=\left\{\check{\mathfrak{g}}_{1}, \check{\mathfrak{g}}_{2}, \check{\mathfrak{g}}_{3}, \check{\mathfrak{g}}_{4}\right\}$ be the group of four senior specialists/decision experts with weight vector $\hat{\boldsymbol{D}}^{\prime}=(0.1,0.2,0.3,0.4)$, where $\check{\mathfrak{g}}_{1}=(0.7,0.1), \check{\mathfrak{g}}_{2}=(0.5,0.7), \mathfrak{g}_{3}=(0.8,0.4)$ and $\check{\mathfrak{g}}_{4}=(0.2,0.3)$. Here we have four q́-rung orthopair fuzzy numbers, $\widetilde{\Xi}_{1}=(0.78,0.45), \widetilde{\Xi}_{2}=(0.32,0.56), \widetilde{\Xi}_{3}=(0.67,0.33)$, and $\widetilde{\Xi}_{4}=(0.87,0.21)$ with associated weight vector $\hat{\hat{\omega}}=(0.4,0.3,0.2,0.1)$, here $\mathfrak{q}=4$, then

$$
\sqrt[\mathfrak{q}]{\widetilde{\prod}_{z=1}^{\mathfrak{q}}\left(\breve{\Theta}_{\mathfrak{g} z}^{\hat{\omega}_{z}^{\prime}}\right)^{\mathfrak{q}}+\widetilde{\prod}_{\mathfrak{i}=1}^{\mathfrak{n}}\left(\breve{\Theta}_{\mathfrak{i}}^{\hat{\omega}_{\mathfrak{i}}}\right)^{\mathfrak{q}}-\widetilde{\prod}_{z=1}^{\mathfrak{q}}\left(\breve{\Theta}_{\mathfrak{\mathfrak { g }} z}^{\hat{\omega}_{z}^{\prime}}\right) \cdot \widetilde{\mathfrak{T}}^{\mathfrak{q}} \cdot \widetilde{\prod}_{\mathfrak{i}=1}^{\mathfrak{n}}\left(\breve{\Theta}_{\mathfrak{i}}^{\hat{\omega}_{\mathfrak{i}}}\right)^{\mathfrak{q}}}=0.615040
$$

In addition,

$$
\sqrt[\mathfrak{q}]{1-\widetilde{\prod}_{z=1}^{\mathfrak{q}}\left(1-\left(\mathfrak{K}_{\mathfrak{g}_{z}}\right)^{\mathfrak{q}}\right)^{\omega_{z}^{\prime}}} \cdot \sqrt[\mathfrak{q}]{1-\widetilde{\prod}_{\mathfrak{i}=1}^{\mathfrak{n}}\left(1-\left(\mathfrak{K}_{\mathfrak{i}}\right)^{\mathfrak{q}}\right)^{\hat{\omega}_{\mathfrak{i}}}}=0.236624
$$

By Theorem 4.2, we have 


$$
\begin{aligned}
& \operatorname{GGQROFWG(}\left(\left(\widetilde{\Xi}_{1}, \widetilde{\Xi}_{2}, \widetilde{\Xi}_{3}, \widetilde{\Xi}_{4}\right),\left(\check{\mathfrak{g}}_{1}, \check{\mathfrak{g}}_{2}, \check{\mathfrak{g}}_{3}, \check{\mathfrak{g}}_{4}\right)\right)=\mathfrak{\mathfrak { q }}-\operatorname{ROFWG}\left(\check{\mathfrak{g}}_{1}, \check{\mathfrak{g}}_{2}, \check{\mathfrak{g}}_{3}, \check{\mathfrak{g}}_{4}\right) \otimes \mathfrak{\mathfrak { q }}-R O F W G\left(\widetilde{\Xi}_{1}, \widetilde{\Xi}_{2}, \widetilde{\Xi}_{3}, \widetilde{\Xi}_{4}\right) \\
& =\left(\sqrt[\mathfrak{q}]{\widetilde{\prod}_{z=1}^{\mathfrak{q}}\left(\breve{\Theta}_{\mathfrak{\mathfrak { g }}_{z}}^{\hat{\omega}_{z}^{\prime}}\right)^{\mathfrak{q}}+\widetilde{\prod}_{\mathfrak{i}=1}^{\mathfrak{n}}\left(\breve{\Theta}_{\mathfrak{i}}^{\hat{\omega}_{\mathfrak{i}}}\right)^{\mathfrak{q}}-\widetilde{\prod}_{z=1}^{\mathfrak{q}}\left(\breve{\Theta}_{\breve{\mathfrak{g}}_{z}}^{\hat{\omega}_{z}^{\prime}}\right)^{\mathfrak{q}} \cdot \widetilde{\prod}_{\mathfrak{i}=1}^{\mathfrak{n}}\left(\breve{\Theta}_{i}^{\hat{\omega}_{\mathfrak{i}}}\right)^{\mathfrak{q}}},\right. \\
& \left.\sqrt[\mathfrak{q}]{1-\widetilde{\prod}_{z=1}^{\mathfrak{q}}\left(1-\left(\mathfrak{K}_{\check{\mathfrak{g}}_{z}}\right)^{\mathfrak{q}}\right)^{\hat{\omega}_{z}^{\prime}}} \cdot \sqrt[\mathfrak{q}]{1-\widetilde{\prod}_{\mathfrak{i}=1}^{\mathfrak{n}}\left(1-\left(\mathfrak{K}_{\mathfrak{i}}\right)^{\mathfrak{q}}\right)^{\hat{\omega}_{\mathfrak{i}}}}\right) \\
& =(0.615040,0.236624)
\end{aligned}
$$

Proposition 27. Let $\widetilde{\Xi}_{\mathfrak{i}}=\left(\breve{\Theta}_{\mathfrak{i}}, \mathfrak{K}_{\mathfrak{i}}\right)(\mathfrak{i}=1,2, \ldots, \mathfrak{n})$ be a set of $\mathfrak{\mathfrak { q }}$-ROFNs, there are $\mathfrak{\mathfrak { q }}$ specialists/decision experts to verify the $\mathfrak{\mathfrak { q }}$-ROF information. If $\check{\mathfrak{g}}_{z}=\left(\breve{\Theta}_{\mathfrak{g}_{z}}, \mathfrak{K}_{\mathfrak{\mathfrak { g }}_{z}}\right)(\mathfrak{i}=1,2, \ldots, \mathfrak{q})$ be the specialists/decision experts for the $\mathfrak{q}-R O F N s \widetilde{\Xi}_{\mathfrak{i}}$, then the GGQROFWG-operator has the given characteristics:

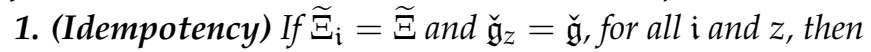

$$
\operatorname{GGQROFWG}\left(\left(\widetilde{\Xi}_{1}, \widetilde{\Xi}_{2}, \ldots, \widetilde{\Xi}_{\mathfrak{n}}\right),\left(\check{\mathfrak{g}}_{1}, \check{\mathfrak{g}}_{2}, \ldots, \check{\mathfrak{g}}_{\mathfrak{q}}\right)\right)=\check{\mathfrak{g}} \otimes \widetilde{\Xi}
$$

2. (Monotonicity) Let $\widetilde{\Xi}_{\mathfrak{i}}^{\star}=\left(\breve{\Theta}_{\mathfrak{i}}^{\star}, \mathfrak{K}_{\mathfrak{i}}^{\star}\right)(\mathfrak{i}=1,2, \ldots, \mathfrak{n})$ be a set of $\mathfrak{\mathfrak { q }}-$ ROFNs such that $\mathfrak{K}_{\mathfrak{i}}^{\star} \leq \mathfrak{K}_{\mathfrak{i}}$ and $\breve{\Theta}_{\mathfrak{i}} \leq \breve{\Theta}_{\mathfrak{i}}^{\star}$ for all $\mathrm{i}$, then

$$
\operatorname{GGQROFWG}\left(\left(\widetilde{\Xi}_{1}, \widetilde{\Xi}_{2}, \ldots, \widetilde{\Xi}_{\mathfrak{n}}\right),\left(\check{\mathfrak{g}}_{1}, \check{\mathfrak{g}}_{2}, \ldots, \check{\mathfrak{g}}_{\mathfrak{q}}\right)\right) \leq \operatorname{GGQROFWG}\left(\left(\widetilde{\Xi}_{1}^{\star}, \widetilde{\Xi}_{2}^{\star}, \ldots, \widetilde{\Xi}_{\mathfrak{n}}^{\star}\right),\left(\check{\mathfrak{g}}_{1}, \check{\mathfrak{g}}_{2}, \ldots, \check{\mathfrak{g}}_{\mathfrak{q}}\right)\right)
$$

3. (Commutativity) Let $\widetilde{\Xi}_{\mathfrak{i}}=\left(\breve{\Theta}_{\mathfrak{i}}, \mathfrak{K}_{\mathfrak{i}}\right)$ and $\widetilde{\Xi}_{\mathfrak{i}}^{*}=\left(\mathfrak{K}_{\mathfrak{i}}{ }^{*}, \breve{\Theta}_{\mathfrak{i}}^{*}\right)(\mathfrak{i}=1,2, \ldots, \mathfrak{n})$ be two sets of $\mathfrak{n} \mathfrak{q}-$ ROFNs such that $\widetilde{\Xi}_{\mathfrak{i}}^{*}$ is any permutation of $\widetilde{\Xi}_{\mathfrak{i}}$, then

$$
\operatorname{GGQROFWG}\left(\left(\widetilde{\Xi}_{1}, \widetilde{\Xi}_{2}, \ldots, \widetilde{\Xi}_{\mathfrak{n}}\right),\left(\check{\mathfrak{g}}_{1}, \check{\mathfrak{g}}_{2}, \ldots, \check{\mathfrak{g}}_{\mathfrak{q}}\right)\right)=\operatorname{GGQROFWG}\left(\left(\widetilde{\Xi}_{1}^{*}, \widetilde{\Xi}_{2}^{*}, \ldots, \widetilde{\Xi}_{\mathfrak{n}}^{*}\right),\left(\check{\mathfrak{g}}_{1}, \check{\mathfrak{g}}_{2}, \ldots, \check{\mathfrak{g}}_{\mathfrak{q}}\right)\right)
$$

4. If the preference of another decision maker to the assessed object is considered to be $\check{\mathfrak{g}}_{z}=(0,1)$ for all $z$, then the GGQROFWG operator becomes the q́-ROFWG-operator.

5. If the preference of another decision maker to the assessed object is considered to be $\check{\mathfrak{g}}_{z}=(1,0)$ for all $z$, then the GGQROFWG operator provides the value $(1,0)$.

Proof. Here we leave proof.

\subsection{Group-Generalized q́-ROF Ordered Weighted Geometric Operator}

Definition 28. Suppose there are $\mathfrak{q}$ specialists/decision experts to verify the $\mathfrak{q}-R O F$ information. Let $\breve{\mathfrak{g}}_{z}=\left(\breve{\Theta}_{\breve{\mathfrak{g}}_{z}}, \mathfrak{K}_{\mathfrak{\mathfrak { g }}_{z}}\right)(\mathfrak{i}=1,2, \ldots, \mathfrak{\mathfrak { q }})$ be the specialists/decision experts for the $\mathfrak{\mathfrak { q }}-$ ROFNs $\widetilde{\Xi}_{\mathfrak{i}}=$ $\left(\breve{\Theta}_{\mathfrak{i}}, \mathfrak{K}_{\mathfrak{i}}\right)(\mathfrak{i}=1,2, \ldots, \mathfrak{n})$, then the GGQROFOWG-operator is described as,

$$
\operatorname{GGQROFOWG}\left(\left(\widetilde{\Xi}_{1}, \widetilde{\Xi}_{2}, \ldots, \widetilde{\Xi}_{\mathfrak{n}}\right),\left(\check{\mathfrak{g}}_{1}, \check{\mathfrak{g}}_{2}, \ldots, \check{\mathfrak{g}}_{\mathfrak{q}}\right)\right)=\mathfrak{q}-\operatorname{ROFWG}\left(\check{\mathfrak{g}}_{1}, \check{\mathfrak{g}}_{2}, \ldots, \check{\mathfrak{g}}_{\mathfrak{q}}\right) \otimes \mathfrak{q}-R O F O W G\left(\widetilde{\Xi}_{1}, \widetilde{\Xi}_{2}, \ldots, \widetilde{\Xi}_{\mathfrak{n}}\right)
$$

Theorem 29. Let $\mathfrak{q}$ be the number of specialists/decision experts to verify the $\mathfrak{q}$-ROF information. Let $\check{\mathfrak{g}}_{z}=\left(\breve{\Theta}_{\check{\mathfrak{g}}_{z}}, \mathfrak{K}_{\check{\mathfrak{g}}_{z}}\right)(\mathfrak{i}=1,2, \ldots, \mathfrak{\mathfrak { q }})$ be the specialists/decision experts for the $\mathfrak{\mathfrak { q }}-R O F N s \widetilde{\Xi}_{\mathfrak{i}}=\left(\breve{\Theta}_{\mathfrak{i}}, \mathfrak{K}_{\mathfrak{i}}\right)$ $(\mathfrak{i}=1,2, \ldots, \mathfrak{n}) \cdot \omega^{\prime}=\left(\hat{\omega}_{1}^{\prime}, \hat{\omega}_{2}^{\prime}, \ldots, \hat{\omega}_{\mathfrak{q}}^{\prime}\right)^{T}, \hat{\omega}=\left(\hat{\omega}_{1}, \hat{\omega}_{2}, \ldots, \omega_{\mathfrak{n}}\right)^{T}$ are the weight vectors of specialists/decision makers and $\widetilde{\Xi}_{\mathfrak{i}}$ respectively and $\hat{\omega}_{\mathfrak{i}}^{\prime} \in[0,1], \sum_{\mathfrak{i}=1}^{\mathfrak{q}} \hat{\omega}_{\mathfrak{i}}^{\prime}=1, \hat{\omega}_{\mathfrak{i}} \in[0,1], \sum_{\mathfrak{i}=1}^{\mathfrak{n}} \hat{\omega}_{\mathfrak{i}}=1$, then the GGQROFOWG-operator is described as

$$
\begin{aligned}
& \text { GGQROFOWG }\left(\left(\widetilde{\Xi}_{1}, \widetilde{\Xi}_{2}, \ldots, \widetilde{\Xi}_{\mathfrak{n}}\right),\left(\check{\mathfrak{g}}_{1}, \check{\mathfrak{g}}_{2}, \ldots, \check{\mathfrak{g}}_{\mathfrak{q}}\right)\right)=\mathfrak{q}-R O F W G\left(\check{\mathfrak{g}}_{1}, \check{\mathfrak{g}}_{2}, \ldots, \check{\mathfrak{g}}_{\mathfrak{q}}\right) \otimes \mathfrak{q}-R O F O W G\left(\widetilde{\Xi}_{1}, \widetilde{\Xi}_{2}, \ldots, \widetilde{\Xi}_{\mathfrak{n}}\right)
\end{aligned}
$$

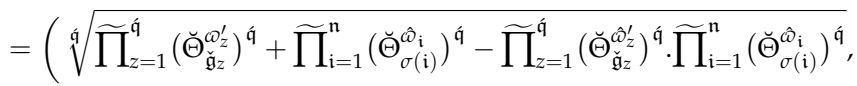

$$
\begin{aligned}
& \left.\sqrt[\mathfrak{q}]{1-\widetilde{\prod}_{z=1}^{\mathfrak{q}}\left(1-\left(\mathfrak{K}_{\mathfrak{\mathfrak { g }}_{z}}\right)^{\mathfrak{q}}\right)^{\hat{\omega}_{z}^{\prime}}} \cdot \sqrt[\mathfrak{q}]{1-\widetilde{\prod}_{\mathfrak{i}=1}^{\mathfrak{n}}\left(1-\left(\mathfrak{K}_{\sigma(\mathfrak{i})}\right)^{\mathfrak{q}}\right)^{\hat{\omega}_{\mathfrak{i}}}}\right)
\end{aligned}
$$


$(\sigma(1), \sigma(2), \ldots, \sigma(\mathfrak{n}))$ is a permutation of $(1,2, \ldots, \mathfrak{n})$, such that $\widetilde{\Xi}_{\sigma(\mathfrak{i}-1)} \geq \widetilde{\Xi}_{\sigma(\mathfrak{i})}$ for any $\mathfrak{i}$.

Proof. Proof is same as Theorem 4.2.

Example 30. Let $\check{\mathfrak{g}}_{z}=\left\{\check{\mathfrak{g}}_{1}, \mathfrak{g}_{2}, \check{\mathfrak{g}}_{3}, \check{\mathfrak{g}}_{4}\right\}$ be the group of four senior specialists/decision experts with weight vector $\hat{\boldsymbol{\omega}}^{\prime}=(0.1,0.2,0.3,0.4)$, where $\check{\mathfrak{g}}_{1}=(0.7,0.1), \mathfrak{g}_{2}=(0.5,0.7), \check{\mathfrak{g}}_{3}=(0.8,0.4)$ and $\check{\mathfrak{g}}_{4}=(0.2,0.3)$. Here we have four q́-rung orthopair fuzzy numbers. $\widetilde{\Xi}_{1}=(0.78,0.45), \widetilde{\Xi}_{2}=(0.32,0.56), \widetilde{\Xi}_{3}=(0.67,0.33)$, and $\widetilde{\Xi}_{4}=(0.87,0.21)$ with associated weight vector $\mathcal{O}=(0.4,0.3,0.2,0.1)$. Here $\mathfrak{q}=4$, first we find score functions of all $\widetilde{\Xi}_{i}$.

$$
\begin{aligned}
& \widetilde{\neg}\left(\widetilde{\Xi}_{1}\right)=0.329144 \\
& \widetilde{\urcorner}\left(\widetilde{\Xi}_{2}\right)=-0.087859 \\
& \widetilde{\neg}\left(\widetilde{\Xi}_{3}\right)=0.189652 \\
& \widetilde{\neg}\left(\widetilde{\Xi}_{4}\right)=0.570952
\end{aligned}
$$

On the behalf of score functions, $\widetilde{\Xi}_{\sigma(1)}=\widetilde{\Xi}_{4}, \widetilde{\Xi}_{\sigma(2)}=\widetilde{\Xi}_{1}, \widetilde{\Xi}_{\sigma(3)}=\widetilde{\Xi}_{3}$, and $\widetilde{\Xi}_{\sigma(4)}=\widetilde{\Xi}_{2}$, then

$$
\sqrt[\mathfrak{q}]{\widetilde{\prod}_{z=1}^{\mathfrak{q}}\left(\breve{\Theta}_{\mathfrak{\mathfrak { g }}_{z}}^{\hat{\omega}_{z}^{\prime}}\right)^{\mathfrak{q}}+\widetilde{\prod}_{\mathfrak{i}=1}^{\mathfrak{n}}\left(\breve{\Theta}_{\sigma(\mathfrak{i})}^{\omega_{\mathfrak{i}}}\right)^{\mathfrak{\mathfrak { q }}}-\widetilde{\prod}_{z=1}^{\mathfrak{q}}\left(\breve{\Theta}_{\mathfrak{\mathfrak { g }}_{z}}^{\hat{\omega}_{z}^{\prime}}\right)^{\mathfrak{q}} \cdot \widetilde{\prod}_{\mathfrak{i}=1}^{\mathfrak{n}}\left(\breve{\Theta}_{\sigma(\mathfrak{i})}^{\hat{\omega}_{\mathfrak{i}}}\right)^{\mathfrak{q}}}=0.424947
$$

In addition,

$$
\sqrt[\mathfrak{q}]{1-\widetilde{\prod}_{z=1}^{\mathfrak{q}}\left(1-\left(\mathfrak{K}_{\check{\mathfrak{g}}_{z}}\right) \mathfrak{\mathfrak { q }}\right) \hat{\mathfrak{\omega}}_{z}^{\prime}} \cdot \sqrt[\mathfrak{q}]{1-\widetilde{\prod}_{\mathfrak{i}=1}^{\mathfrak{n}}\left(1-\left(\mathfrak{K}_{\sigma(\mathfrak{i})}\right)^{\mathfrak{q}}\right)^{\hat{\mathfrak{\omega}}_{\mathfrak{i}}}}=0.201330
$$

By Theorem 4.6, we have

$$
\begin{aligned}
& \text { GGQROFOWG }\left(\left(\widetilde{\Xi}_{1}, \widetilde{\Xi}_{2}, \widetilde{\Xi}_{3}, \widetilde{\Xi}_{4}\right),\left(\check{\mathfrak{g}}_{1}, \check{\mathfrak{g}}_{2}, \check{\mathfrak{g}}_{3}, \check{\mathfrak{g}}_{4}\right)\right)=\mathfrak{\mathfrak { q }}-\operatorname{ROFWG}\left(\mathfrak{g}_{1}, \check{\mathfrak{g}}_{2}, \check{\mathfrak{g}}_{3}, \check{\mathfrak{g}}_{4}\right) \otimes \mathfrak{q}-R O F O W G\left(\widetilde{\Xi}_{1}, \widetilde{\Xi}_{2}, \widetilde{\Xi}_{3}, \widetilde{\Xi}_{4}\right)
\end{aligned}
$$

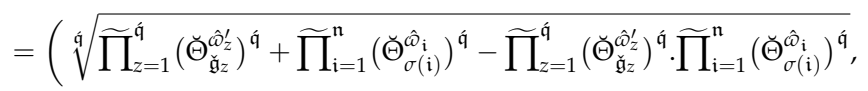

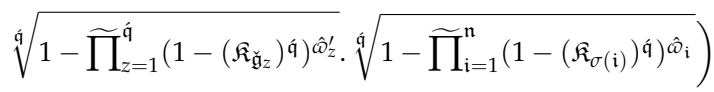

$$
\begin{aligned}
& =(0.424947,0.201330)
\end{aligned}
$$

Proposition 31. Let $\widetilde{\Xi}_{\mathfrak{i}}=\left(\breve{\Theta}_{\mathfrak{i}}, \mathfrak{K}_{\mathfrak{i}}\right)(\mathfrak{i}=1,2, \ldots, \mathfrak{n})$ be a set of $\mathfrak{\mathfrak { q }}$-ROFNs, there are $\mathfrak{\mathfrak { q }}$ specialists/decision to verify the $\mathfrak{\mathfrak { q }}$-ROF information. Let $\check{\mathfrak{g}}_{z}=\left(\breve{\Theta}_{\mathfrak{g}_{z}}, \mathfrak{K}_{\mathfrak{g}_{z}}\right)(\mathfrak{i}=1,2, \ldots, \mathfrak{q})$ be the specialists/decision for the $\mathfrak{\mathfrak { q }}-R O F N s$ $\widetilde{\Xi}_{i}$, then the GGQROFOWG-operator has the given characteristics:

1. (Idempotency) If $\widetilde{\Xi}_{\mathfrak{i}}=\check{\Xi}$ and $\check{\mathfrak{g}}_{z}=\breve{\mathfrak{g}}$, for all $\mathfrak{i}$ and $z$ then

$$
\operatorname{GGQROFOWG}\left(\left(\widetilde{\Xi}_{1}, \widetilde{\Xi}_{2}, \ldots, \widetilde{\Xi}_{\mathfrak{n}}\right),\left(\check{\mathfrak{g}}_{1}, \check{\mathfrak{g}}_{2}, \ldots, \check{\mathfrak{g}}_{\mathfrak{q}}\right)\right)=\check{\mathfrak{g}} \otimes \widetilde{\Xi}
$$

2. (Monotonicity) Let $\widetilde{\Xi}_{\mathfrak{i}}^{\star}=\left(\breve{\Theta}_{\mathfrak{i}}^{\star}, \mathfrak{K}_{\mathfrak{i}}^{\star}\right)(\mathfrak{i}=1,2, \ldots, \mathfrak{n})$ be a set of $\mathfrak{q}-$ ROFNs such that $\mathfrak{K}_{\mathfrak{i}}^{\star} \leq \mathfrak{K}_{\mathfrak{i}}$ and $\breve{\Theta}_{\mathfrak{i}} \leq \breve{\Theta}_{\mathfrak{i}}^{\star}$ for all $i$, then

$\operatorname{GGQROFOWG}\left(\left(\widetilde{\Xi}_{1}, \widetilde{\Xi}_{2}, \ldots, \widetilde{\Xi}_{\mathfrak{n}}\right),\left(\check{\mathfrak{g}}_{1}, \check{\mathfrak{g}}_{2}, \ldots, \check{\mathfrak{g}}_{\mathfrak{q}}\right)\right) \leq \operatorname{GGQROFOWG}\left(\left(\widetilde{\Xi}_{1}^{\star}, \widetilde{\Xi}_{2}^{\star}, \ldots, \widetilde{\Xi}_{\mathfrak{n}}^{\star}\right),\left(\check{\mathfrak{g}}_{1}, \check{\mathfrak{g}}_{2}, \ldots, \check{\mathfrak{g}}_{\mathfrak{q}}\right)\right)$

3. (Commutativity) Let $\widetilde{\Xi}_{\mathfrak{i}}=\left(\breve{\Theta}_{\mathfrak{i}}, \mathfrak{K}_{\mathfrak{i}}\right)(\mathfrak{i}=1,2, \ldots, \mathfrak{n})$ and $\widetilde{\Xi}_{\mathfrak{i}}^{*}=\left(\breve{\Theta}_{\mathfrak{i}}^{*}, \mathfrak{K}_{\mathfrak{i}}{ }^{*}\right)(\mathfrak{i}=1,2, \ldots, \mathfrak{n})$ be a two collection of $n \mathfrak{q}-R O F N$ such that $\widetilde{\Xi}_{\mathfrak{i}}^{*}$ is any permutation of $\widetilde{\Xi}_{\mathfrak{i}}$, then

$\operatorname{GGQROFOWG}\left(\left(\widetilde{\Xi}_{1}, \widetilde{\Xi}_{2}, \ldots, \widetilde{\Xi}_{\mathfrak{n}}\right),\left(\check{\mathfrak{g}}_{1}, \check{\mathfrak{g}}_{2}, \ldots, \check{\mathfrak{g}}_{\mathfrak{q}}\right)\right)=\operatorname{GGQROFOWG}\left(\left(\widetilde{\Xi}_{1}^{*}, \widetilde{\Xi}_{2}^{*}, \ldots, \widetilde{\Xi}_{\mathfrak{n}}^{*}\right),\left(\check{\mathfrak{g}}_{1}, \check{\mathfrak{g}}_{2}, \ldots, \check{\mathfrak{g}}_{\mathfrak{q}}\right)\right)$ 
4. If the preference of the specialists/decision experts to the assessed object is considered to be $\check{\mathfrak{g}}_{z}=(0,1)$ for all $z$, then the GGQROFOWG-operator becomes the $\mathfrak{q}$-ROFOWG-operator.

5. If the preference of another decision maker to the assessed object is considered to be $\check{\mathfrak{g}}_{z}=(1,0)$ for all $z$, then the GGQROFOWG-operator provides the value $(1,0)$.

Proof. Here we leave the proof.

\subsection{Group-Generalized q́-ROF Hybrid Geometric Operator}

Definition 32. Suppose there are $\mathfrak{q}$ specialists/decision experts to verify the $\mathfrak{\mathfrak { q }}$-ROF information. Let $\check{\mathfrak{g}}_{z}=$ $\left(\breve{\Theta}_{\check{\mathfrak{g}}_{z}}, \mathfrak{K}_{\check{\mathfrak{g}}_{z}}\right)(\mathfrak{i}=1,2, \ldots, \mathfrak{\mathfrak { q }})$ be the specialists/decision experts for the $\mathfrak{\mathfrak { q }}-R O F N s \widetilde{\Xi}_{\mathfrak{i}}=\left(\breve{\Theta}_{\mathfrak{i}}, \mathfrak{K}_{\mathfrak{i}}\right)(\mathfrak{i}=1,2, \ldots, \mathfrak{n})$, then the GGQROFHG-operator is described as,

$\operatorname{GGQROFHG}\left(\left(\widetilde{\Xi}_{1}, \widetilde{\Xi}_{2}, \ldots, \widetilde{\Xi}_{\mathfrak{n}}\right),\left(\check{\mathfrak{g}}_{1}, \check{\mathfrak{g}}_{2}, \ldots, \check{\mathfrak{g}}_{\mathfrak{q}}\right)\right)=\mathfrak{\mathfrak { q }}-\operatorname{ROFWG}\left(\check{\mathfrak{g}}_{1}, \check{\mathfrak{g}}_{2}, \ldots, \mathfrak{g}_{\mathfrak{q}}\right) \otimes \mathfrak{\mathfrak { q }}-R O F H G\left(\widetilde{\Xi}_{1}, \widetilde{\Xi}_{2}, \ldots, \widetilde{\Xi}_{\mathfrak{n}}\right)$

Theorem 33. Let there be q́ specialists/decision experts to verify the $\mathfrak{\mathfrak { q }}$-ROF information. Let $\mathfrak{g}_{z}=\left(\breve{\Theta}_{\check{\mathfrak{g}}_{z}}, \mathfrak{\mathfrak { K }}_{\check{\mathfrak{g}}_{z}}\right)$ $(\mathfrak{i}=1,2, \ldots, \mathfrak{q})$ be the specialists/decision experts for the $\mathfrak{q}-R O F N s \widetilde{\Xi}_{\mathfrak{i}}=\left(\breve{\Theta}_{\mathfrak{i}}, \mathfrak{K}_{\mathfrak{i}}\right)(\mathfrak{i}=1,2, \ldots, \mathfrak{n}), \hat{\boldsymbol{\omega}}^{\prime}=$ $\left(\hat{\omega}_{1}^{\prime}, \hat{\omega}_{2}^{\prime}, \ldots, \hat{\omega}_{\hat{q}}^{\prime}\right)^{T}$, and $\hat{\omega}=\left(\hat{\omega}_{1}, \hat{\omega}_{2}, \ldots, \omega_{\mathfrak{n}}\right)^{T}$ are the weight vectors of specialists/observers and $\widetilde{\Xi}_{\mathfrak{i}}$, respectively and $\hat{\omega}_{\mathfrak{i}}^{\prime} \in[0,1], \sum_{\mathfrak{i}=1}^{\mathfrak{q}} \omega_{\mathfrak{i}}^{\prime}=1, \hat{\omega}_{\mathfrak{i}} \in[0,1]$, and $\sum_{\mathfrak{i}=1}^{\mathfrak{n}} \hat{\omega}_{\mathfrak{i}}=1$, then GGQROFHG-operator is described as

$$
\begin{aligned}
& \operatorname{GGQROFHG}\left(\left(\widetilde{\Xi}_{1}, \widetilde{\Xi}_{2}, \ldots, \widetilde{\Xi}_{\mathfrak{n}}\right),\left(\mathfrak{g}_{1}, \check{\mathfrak{g}}_{2}, \ldots, a a \check{\mathfrak{g}}_{\mathfrak{q}}\right)\right)=\mathfrak{\mathfrak { q }}-R O F W G\left(\check{\mathfrak{g}}_{1}, \check{\mathfrak{g}}_{2}, \ldots, \check{\mathfrak{g}}_{\mathfrak{q}}\right) \otimes \mathfrak{q}-R O F H G\left(\widetilde{\Xi}_{1}, \widetilde{\Xi}_{2}, \ldots, \widetilde{\Xi}_{\mathfrak{n}}\right)
\end{aligned}
$$

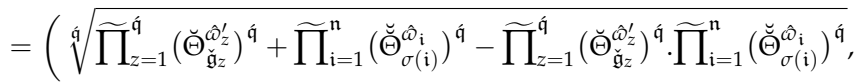

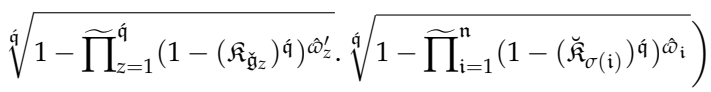

Here $\breve{\Xi}_{\mathfrak{i}}=\mathfrak{n} \widetilde{\xi}_{\mathfrak{i}} \widetilde{\Xi}_{\mathfrak{i}}, n$ is the number of $\mathfrak{q}$-ROFNs, $\xi_{\mathfrak{i}}$ is a standard weight vector of $\widetilde{\Xi}_{\mathfrak{i}}$, and $(\sigma(1), \sigma(2), \ldots, \sigma(\mathfrak{n}))$ is a permutation of $(1,2, \ldots, \mathfrak{n})$, such that $\breve{\Xi}_{\sigma(\mathfrak{i}-1)} \geq \breve{\Xi}_{\sigma(\mathfrak{i})}$ for any $\mathfrak{i}$.

Proof. Proof is the same as Theorem 4.2.

Example 34. Let $\check{\mathfrak{g}}_{z}=\left\{\check{\mathfrak{g}}_{1}, \check{\mathfrak{g}}_{2}, \mathfrak{g}_{3}, \check{\mathfrak{g}}_{4}\right\}$ be the group of four senior specialists/decision experts with weight vector $\hat{\boldsymbol{D}}^{\prime}=(0.1,0.2,0.3,0.4)$, where $\check{\mathfrak{g}}_{1}=(0.7,0.1), \mathfrak{g}_{2}=(0.5,0.7), \check{\mathfrak{g}}_{3}=(0.8,0.4)$, and $\check{\mathfrak{g}}_{4}=(0.2,0.3)$. Here we have four q́-rung orthopair fuzzy numbers. $\widetilde{\Xi}_{1}=(0.78,0.45), \widetilde{\Xi}_{2}=(0.32,0.56), \widetilde{\Xi}_{3}=(0.67,0.33)$, and $\widetilde{\Xi}_{3}=(0.67,0.33)$ with associated weight vector $\hat{\omega}=(0.4,0.3,0.2,0.1)$. Here $\hat{\mathfrak{q}}=4$ and a standard weight vector will be $\xi_{\mathfrak{i}}=(0.2,0.2,0.3,0.3)$. First we find $\breve{\Xi}_{\mathfrak{i}}=n \widetilde{\xi}_{\mathfrak{i}} \widetilde{\Xi}_{\mathfrak{i}}$ for each $\widetilde{\Xi}_{\mathfrak{i}}$, then we find score functions of each $\breve{\widetilde{\Xi}}_{\mathfrak{i}}$.

$$
\begin{aligned}
& \breve{\Xi}_{1}=(0.745657,0.527922) \\
& \breve{\Xi}_{2}=(0.302716,0.628854) \\
& \breve{\Xi}_{3}=(0.697473,0.264372) \\
& \breve{\Xi}_{4}=(0.894329,0.153696)
\end{aligned}
$$

The score function will be,

$$
\begin{aligned}
& \widetilde{\neg}\left(\breve{\Xi}_{1}\right)=0.231466 \\
& \widetilde{\urcorner}\left(\breve{\Xi}_{2}\right)=-0.147989 \\
& \widetilde{\neg}\left(\widetilde{\Xi}_{3}\right)=-0.231766
\end{aligned}
$$




$$
\widetilde{\neg}\left(\breve{\Xi}_{3}\right)=0.639160
$$

On the behalf of score functions, $\widetilde{\Xi}_{\sigma(1)}=\breve{\Xi}_{4}, \breve{\Xi}_{\sigma(2)}=\breve{\Xi}_{3}, \widetilde{\Xi}_{\sigma(3)}=\breve{\Xi}_{1}$, and $\widetilde{\Xi}_{\sigma(4)}=\breve{\Xi}_{2}$

$$
\sqrt[\mathfrak{q}]{\widetilde{\prod}_{z=1}^{\mathfrak{q}}\left(\breve{\Theta}_{\mathfrak{g}_{z}}^{\omega_{z}^{\prime}}\right)^{\mathfrak{q}}+\widetilde{\prod}_{\mathfrak{i}=1}^{\mathfrak{n}}}\left(\breve{\Theta}_{\sigma(\mathfrak{i})}^{\hat{\omega}_{i}}\right)^{\mathfrak{q}}-\widetilde{\prod}_{z=1}^{\mathfrak{q}}\left(\breve{\Theta}_{\mathfrak{g}_{z}}^{\hat{\omega}_{z}}\right)^{\mathfrak{q}} \widetilde{\prod}_{\mathfrak{i}=1}^{n}\left(\breve{\Theta}_{\sigma(\mathfrak{i})}^{\hat{\omega}_{i}}\right)^{\mathfrak{q}}=0.287407
$$

In addition,

$$
\sqrt[\mathfrak{q}]{1-\widetilde{\prod}_{z=1}^{\mathfrak{q}}\left(1-\left(\mathfrak{K}_{\mathfrak{g}_{z}}\right) \mathfrak{\mathfrak { q }}\right)^{\hat{\omega}_{z}^{\prime}}} \cdot \sqrt[\mathfrak{q}]{1-\widetilde{\prod}_{\mathfrak{i}=1}^{\mathfrak{n}}\left(1-\left(\mathfrak{K}_{\sigma(\mathfrak{i})}\right)^{\mathfrak{q}}\right)^{\hat{\omega}_{\mathfrak{i}}}}=0.216261
$$

By Theorem 4.10, we have

GGQROFHG $\left(\left(\widetilde{\Xi}_{1}, \widetilde{\Xi}_{2}, \widetilde{\Xi}_{3}, \widetilde{\Xi}_{4}\right),\left(\check{\mathfrak{g}}_{1}, \check{\mathfrak{g}}_{2}, \check{\mathfrak{g}}_{3}, \check{\mathfrak{g}}_{4}\right)\right)=\mathfrak{q}-R O F W G\left(\check{\mathfrak{g}}_{1}, \check{\mathfrak{g}}_{2}, \check{\mathfrak{g}}_{3}, \check{\mathfrak{g}}_{4}\right) \otimes \mathfrak{q}-R O F H G\left(\widetilde{\Xi}_{1}, \widetilde{\Xi}_{2}, \widetilde{\Xi}_{3}, \widetilde{\Xi}_{4}\right)$

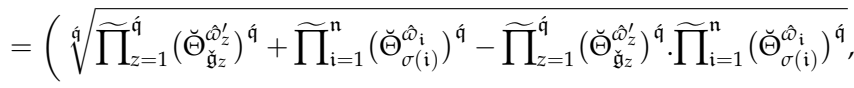

$$
\begin{aligned}
& \left.\sqrt[\mathfrak{q}]{1-\widetilde{\prod}_{z=1}^{\mathfrak{q}}\left(1-\left(\mathfrak{K}_{\mathfrak{g}_{z}}\right) \mathfrak{q}\right) \hat{\omega}_{z}^{\prime}} \cdot \sqrt[\mathfrak{q}]{1-\widetilde{\prod}_{\mathbf{i}=1}^{\mathfrak{n}}\left(1-\left(\mathfrak{K}_{\sigma(\mathfrak{i})}\right)^{\mathfrak{q}}\right)^{\hat{\omega}_{\mathrm{i}}}}\right) \\
& =(0.287407,0.216261)
\end{aligned}
$$

The following observation are taken from the definition of GGQROFHG-operator:

1. If the priorities of the specialists/decision experts to the assessed object are considered to be $\check{\mathfrak{g}}_{z}=(0,1)$ for all $z$, then the GGQROFHG-operator becomes the $\mathfrak{\mathfrak { q }}$-ROFHG-operator.

2. If the priorities of the specialists/decision to the assessed object is considered to be $\check{\mathfrak{g}}_{z}=(1,0)$ for all $z$, then the GQROFHG-operator provides the value $(1,0)$.

3. If $\xi=\left(\frac{1}{n}, \frac{1}{n}, \ldots, \frac{1}{n}\right)^{T}$, then the GGQROFHG-operator reduces to GQROFWG-operator.

4. If $\hat{\omega}=\left(\frac{1}{n}, \frac{1}{n}, \ldots, \frac{1}{n}\right)^{T}$, then the GGQROFHG-operator reduces to GQROFOWG-operator.

\section{Multi-Attribute Decision-Making Method With Application Based On Group-Generalized Parameter}

In this section, the provided method examines MADM challenges, in accordance with proposed aggregation operators. To illustrate the MADM technique efficiently, a numerical demonstration is also discussed in Algorithm 1.

\subsection{Methodology}

Suppose $\Omega=\left\{\widetilde{\mathbb{U}}_{1}, \widetilde{\mathbb{U}}_{2}, \ldots, \widetilde{U}_{m}\right\}$ is a collection of universal elements, $\mathcal{C}=\left\{\curlywedge_{1}, \curlywedge_{2}, \ldots, \curlywedge_{\mathfrak{n}}\right\}$ be a collection of evaluation criteria/attributes and $\hat{\omega}=\left\{\omega_{1}, \hat{\omega}_{2}, \ldots, \hat{\omega}_{\mathfrak{n}}\right\}$ is the weight vector, in a such way that $\hat{\omega}_{j} \in[0,1]$ and $\sum_{j=1}^{n} \hat{\omega}_{j}=1,(j=1,2, \ldots, n)$. A universal element on the evaluation attribute is assessed by the specialist and the assessment values should be in $\mathfrak{q}$-ROFNs. Suppose that $\left(\Omega_{i j}\right)_{m \times n}=\left(\breve{\Theta}_{i j}, \mathfrak{K}_{i j}\right)_{m \times n}$ is a matrix characterized by decision makers. Here $\breve{\Theta}_{i j}$ and $\mathfrak{K}_{i j}$ demonstrates the degree of appreciation and non-appreciation corresponding to alternatives $\widetilde{\cup}_{i}$ to the evaluation attribute $\curlywedge_{j}$ respectively. To make the situation more credible, consider a group of different specialists/decision experts $\partial=\left\{\check{\mathfrak{g}}_{1}, \check{\mathfrak{g}}_{2}, \ldots, \check{\mathfrak{g}}_{\mathfrak{l}}\right\}$ with weight vector $w^{\prime}=\left\{w_{1}^{\prime}, w_{2}^{\prime}, \ldots, w_{\mathfrak{l}}^{\prime}\right\}$ satisfying $w_{k}^{\prime}>0, k=(1,2, \ldots \mathfrak{l})$ and $\sum_{k=1}^{n} w_{\mathfrak{i}}^{\prime}=1$. These decision experts provide their evaluation regarding the priority for each alternative in the terms of $\mathfrak{\mathfrak { q }}$-ROFNs indicated by $\mathfrak{g}_{k}=\left(\breve{\Theta}_{\check{\mathfrak{g}}_{k}}, \mathfrak{K}_{\check{\mathfrak{g}}_{k}}\right)(k=1,2, \ldots, \mathfrak{l})$. To solve the MADM problems, the steps of the algorithm are in the following manner. 


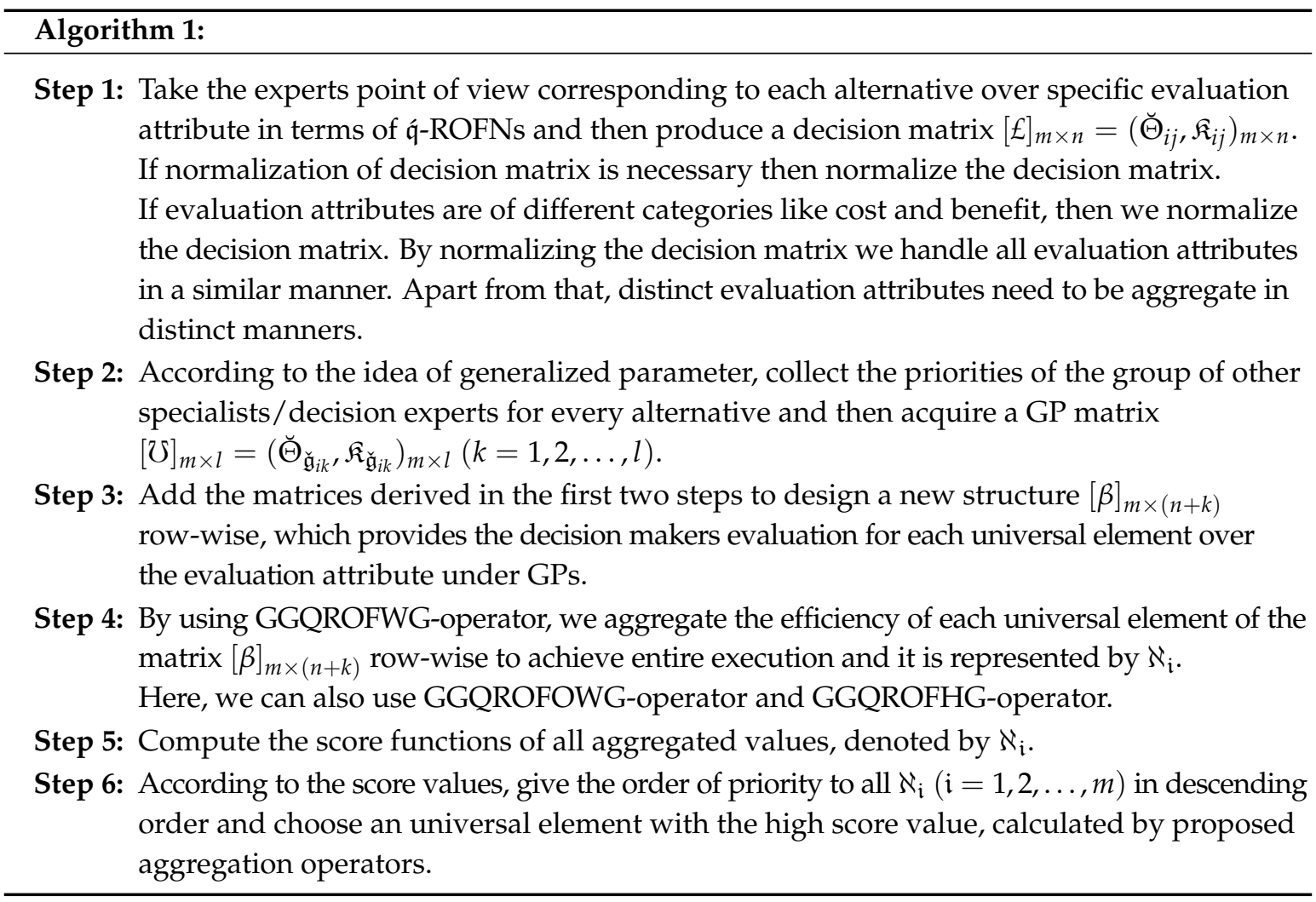

The flow chart of proposed Algorithm is given by Figure 2.

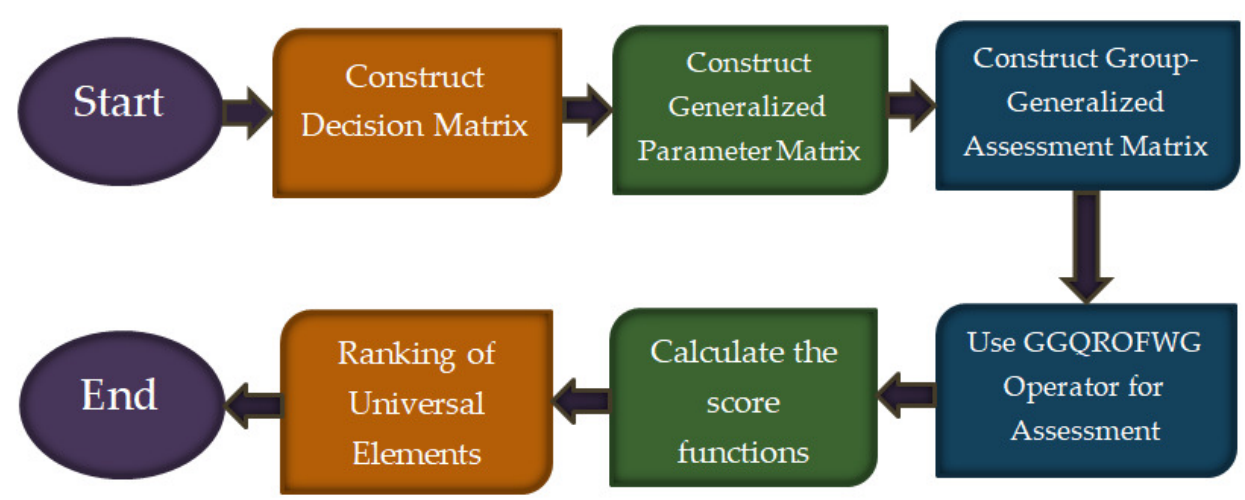

Figure 2. Flow chart of Algorithm.

\subsection{Case Study}

Water is classified among the fundamental life-sustaining needs. If there is no water on the planet earth then life is impossible. However, it is a sad fact that we are not taking appropriate actions for preservation and protection of our natural endowments. Even among the other natural endowments we have, water has the most significance. Currently, Pakistan is facing several problems but possibly the most challenging is the water scarcity. As reported by International Monetary Fund (IMF), Pakistan has 3rd position confronting serious deficiency of water. The requirement of water is escalating, as population of Pakistan is rapidly growing. Therefore, we need more water for agricultural and domestic use. The historical water demands by sector are given in Figure 3. Currently, about 40 percent of Pakistanis do not have availability of fresh water and are influenced by contaminated water mainly polluted by sewerage, pesticides, by fertilizer, and industrial waste water (source: jworldtimes.com). It should be pointed out that while in the 1950s the accessibility of water was nearly $5000 \mathrm{~m}^{3}$ per year, 
it is now reduced to below $1000 \mathrm{~m}^{3}$, that is worldwide limit of shortage of water. From 2009, per annum water reduction is 1500 cubic meters per capita to only 1017 cubic meters (source: tribune.com.pk). The comparison of population and water availability in Pakistan is shown in Figure 4.

One of the main causes of this problem is the lack of actions in water loss management. Due to bad administration and mismanagement, about 30 million acre feet (MAF) of water is wasted. Owing to the fact that, the water accessibility in Pakistan is uniformly decreasing. In May 2018, the "Pakistan Council of Research in water Resources" (PCRWR) declared that, there will be short or no availability of clean water in the country in 2025 (source: jworldtimes.com). The situation of water losses in irrigation systems in Pakistan is shown in Figure 5.

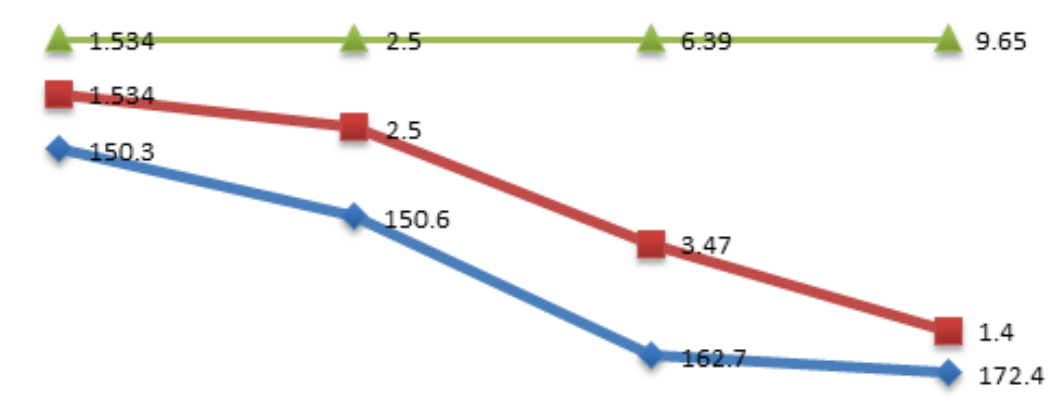

$\begin{array}{cccc}1991 & 2000 & 2008 \\ & & & \\ & & & \\ & & & \end{array}$

Figure 3. Historical water demands (source: www.undp.org).

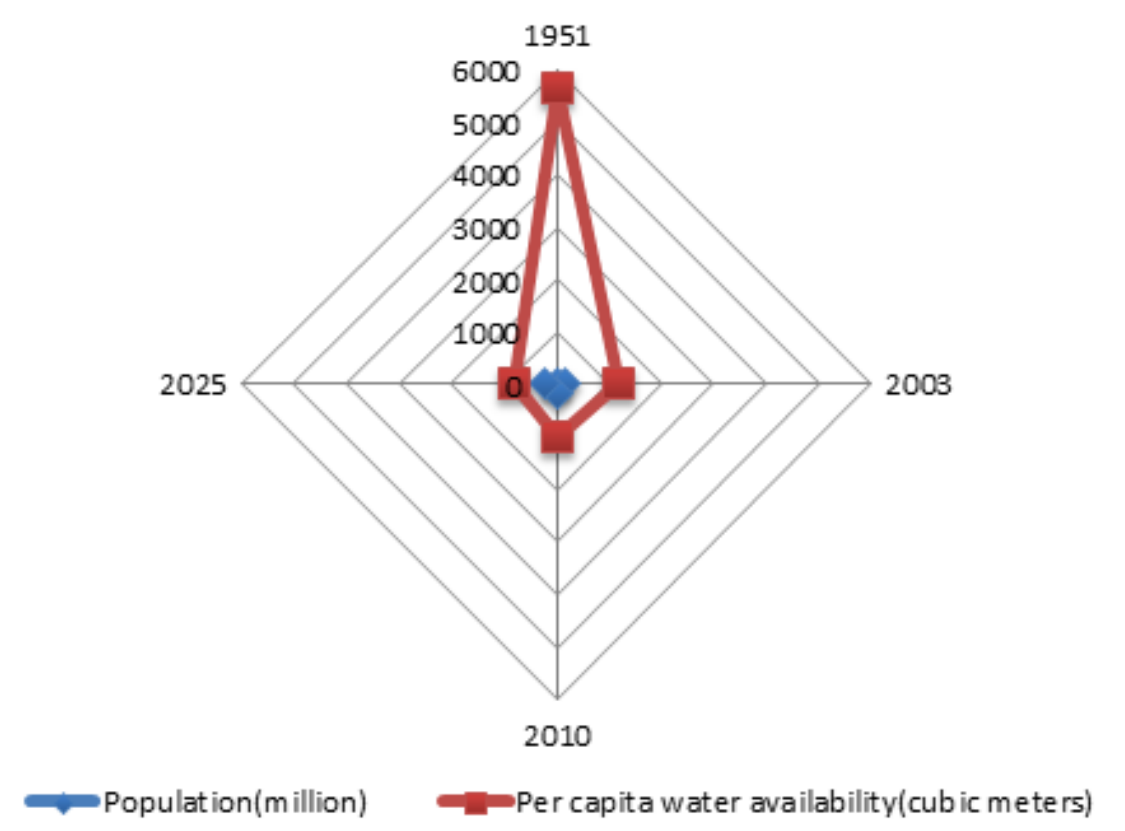

Figure 4. Population vs. water availability in Pakistan (source: pcwr.gov.pk). 


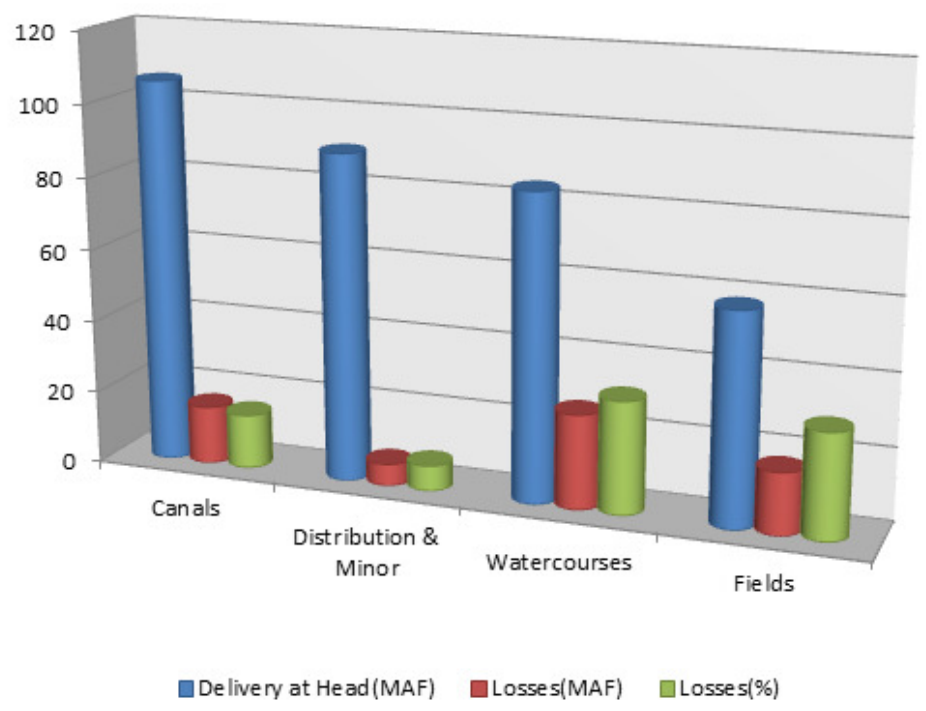

Figure 5. Water losses in an irrigation system (Source: Final PAS water 2019 (pcrwr.gov.pk)).

An adequate and efficient water loss management needs to be considered as a primary objective in improvement of drinkable supply of water. Across the board, policy makers/decision experts need to be aware that any strategy to control water loss in order to be effective must be a continuous activity based on a long term strategy. The success of the strategy will necessarily rely upon the engagement and devotion at every stage throughout the service and obviously the acceptance of suitable policies and methods. The advantages of strategy to control the loss of water could be summarized as follows:

(1) Rescuing an affected and precious expedient.

(2) Growing the effectiveness of available systems.

(3) Retarding enormous financial assets of infrastructure.

(4) Increasing the average life span of the systems.

(5) Increasing the earnings for the service of water.

(6) Reduction of energy demands.

(7) Improvement in Carbon Footprint of the service.

The fundamental goal of this investigation is to construct a comprehensive structure of strategies to recognize and emphasize the suitable strategy to overcome the water loss problem. The selected strategy needs to be able to meet the goals and has compatibility with general policy of water sector particularly ensure the maximum supply, enhancing the water quality, preserving the accessible supply of water. Whereas the administration of losses of water is usually a complicated process of making decisions including various goals and potentials, the concerns of different involved persons as well as the demands of the amendable authorities must take into account to establish a well-organized scheme explicated by efficiently and with clarity. Current situation encompasses the participation of policy makers/decision experts who have a profound knowledge of the decision problem. The preferred strategies were originated from review of the literature on water loss management with specialists and policy makers and on the basis of domestic circumstances of region of interest, as given in Table 1 . The considered strategies are established for water loss management in water distribution network. Particular provisions are usually established in persistent water distribution network, like leakage control. Some of the strategies are closely linked to the situation of recurring supply. To evaluate the efficiency of each strategy, the evaluation attributes (EA) are used. The significance of evaluation attributes need to be clear to recognize the most convenient strategy. The evaluation attributes are derived from review of literature $[5,10]$, as given in Table 2 . 
Table 1. Explanation of strategies.

\begin{tabular}{|c|c|c|}
\hline Code & Strategies & Explanation \\
\hline$\widetilde{\mathbb{U}}_{1}$ & Pressure management & $\begin{array}{l}\text { Managing pressure of system to the highest grade of } \\
\text { service, guarantee the adequate and effective supply, } \\
\text { while reducing useless or excessive pressures }\end{array}$ \\
\hline$\widetilde{U}_{2}$ & Management of assets for service lines & Replacement of mains and affected service lines \\
\hline$\widetilde{\cup}_{3}$ & Enhancing the repairing quality & $\begin{array}{l}\text { To prevent repetition of explosions, } \\
\text { and to minimize the harmful effects } \\
\text { of breakdown of the service }\end{array}$ \\
\hline$\widetilde{\cup}_{4}$ & Monitoring of inefficient use of water service & $\begin{array}{l}\text { Raising awareness of people through beneficial } \\
\text { supervision and campaigning, to eradicate the } \\
\text { improper utilization of water }\end{array}$ \\
\hline$\widetilde{U}_{5}$ & Leakage control & $\begin{array}{l}\text { Adopt measures for identification and repair } \\
\text { of leaks that have not indicated }\end{array}$ \\
\hline$\widetilde{\cup}_{6}$ & Water meters replacements & $\begin{array}{l}\text { Installation of automatic water meters } \\
\text { to eliminate water meters uncertainty }\end{array}$ \\
\hline
\end{tabular}

Table 2. Explanation of evaluation attributes.

\begin{tabular}{|c|c|c|}
\hline Code & Evaluation Attributes & Explanation \\
\hline$\curlywedge_{1}$ & Cost Figure & Related expenses for execution of alternatives \\
\hline$\curlywedge_{2}$ & Benefit Period & Measurement of useful life expectancy of alternative \\
\hline$\iota_{3}$ & Energy Saved & $\begin{array}{l}\text { If the alternative has ability to reduce the utilization } \\
\text { of energy and discharges of green house gas }\end{array}$ \\
\hline$\curlywedge_{4}$ & Supply Reliability & $\begin{array}{l}\text { If the alternative has ability to save a sustained } \\
\text { service and reduce supply hindrances }\end{array}$ \\
\hline$\curlywedge_{5}$ & Flexibility & $\begin{array}{l}\text { If the alternative has capacity of being adjusted to } \\
\text { fulfill different requirements and imprecisions }\end{array}$ \\
\hline
\end{tabular}

\subsection{Numerical Example}

The demonstrative example of water loss management is presented to demonstrate the method. Let $\Omega=\left\{\widetilde{U}_{1}, \widetilde{U}_{2}, \widetilde{U}_{3}, \widetilde{U}_{4}, \widetilde{U}_{5}, \widetilde{U}_{6}\right\}$ be the collection of alternative, $\mathcal{C}=\left\{\curlywedge_{1}, \curlywedge_{2}, \curlywedge_{3}, \curlywedge_{4}, \curlywedge_{5}\right\}$ be the collection of evaluation attributes as given in Table 1 , Table 2 , respectively, $\omega=(0.1,0.1,0.2,0.2,0.4)^{T}$ are the associated weights assigned by different policy makers/decision experts from Pakistan Water and Power Development Authority (WAPDA) and take $\mathfrak{q}=3$. The policy makers/decision makers are asked to give their evaluation in terms of $\mathfrak{q}-$ ROFNs for each strategy against each evaluation criteria.

Step 1: According to the preferences of policy makers/decision experts for each alternative against the distinct evaluation attribute, construct the decision matrix $[£]_{6 \times 5}=\left(\breve{\Theta}_{i j}, \mathfrak{K}_{i j}\right)_{6 \times 5}$, as given in Table 3.

Table 3. q́ -rung orthopair fuzzy decision expert assessment matrix $[£]_{6 \times 5}$.

\begin{tabular}{cccccc}
\hline $\mathbf{\Omega} / \mathcal{C}$ & $\curlywedge_{1}$ & $\curlywedge_{2}$ & $\curlywedge_{3}$ & $\curlywedge_{4}$ & $\curlywedge_{5}$ \\
\hline$\widetilde{U}_{1}$ & $(0.67,0.21)$ & $(0.57,0.11)$ & $(0.86,0.14)$ & $(0.72,0.21)$ & $(0.62,0.21)$ \\
$\widetilde{U}_{2}$ & $(0.35,0.38)$ & $(0.21,0.68)$ & $(0.51,0.66)$ & $(0.27,0.38)$ & $(0.72,0.23)$ \\
$\widetilde{U}_{3}$ & $(0.41,0.17)$ & $(0.35,0.45)$ & $(0.67,0.51)$ & $(0.28,0.78)$ & $(0.72,0.21)$ \\
$\widetilde{U}_{4}$ & $(0.13,0.66)$ & $(0.32,0.31)$ & $(0.35,0.61)$ & $(0.31,0.52)$ & $(0.81,0.24)$ \\
$\widetilde{U}_{5}$ & $(0.67,0.21)$ & $(0.57,0.32)$ & $(0.86,0.14)$ & $(0.70,0.20)$ & $(0.72,0.20)$ \\
$\widetilde{U}_{6}$ & $(0.46,0.38)$ & $(0.32,0.68)$ & $(0.62,0.66)$ & $(0.38,0.28)$ & $(0.81,0.31)$ \\
\hline
\end{tabular}

Step 2: On each strategy, collect the preferences of group of three other specialists/experts of different environmental groups of Pakistan like, Pakistan Environmentalists Association (PEA), Society for conservation and protection of Environment (SCOPE), i.e., with a weight vector $(0.2,0.3,0.5)^{T}$ according to their experiences, that would be helpful in aggregation information. The corresponding generalized parameter matrix $[\mho]_{6 \times 3}=\left(\breve{\Theta}_{\check{\mathfrak{g}}_{i k}}, \mathfrak{K}_{\check{\mathfrak{g}}_{i k}}\right)_{6 \times 3}$. $(k=1,2, \ldots, l)$ is given in Table 4 . 
Table 4. q́ -rung orthopair fuzzy generalized parameter preference matrix $[\mho]_{6 \times 3}$.

\begin{tabular}{cccc}
\hline $\boldsymbol{\Omega} / \check{\partial}$ & $\mathfrak{g}_{1}$ & $\mathfrak{g}_{2}$ & $\mathfrak{g}_{3}$ \\
\hline$\widetilde{\mathbb{U}}_{1}$ & $(0.72,0.25)$ & $(0.68,0.12)$ & $(0.58,0.22)$ \\
$\widetilde{\cup}_{2}$ & $(0.21,0.35)$ & $(0.35,0.63)$ & $(0.82,0.26)$ \\
$\widetilde{\mathbb{U}}_{3}$ & $(0.45,0.26)$ & $(0.35,0.71)$ & $(0.67,0.52)$ \\
$\widetilde{\cup}_{4}$ & $(0.14,0.62)$ & $(0.25,0.14)$ & $(0.38,0.27)$ \\
$\widetilde{\mathbb{U}}_{5}$ & $(0.20,0.17)$ & $(0.31,0.23)$ & $(0.26,0.25)$ \\
$\widetilde{\cup}_{6}$ & $(0.24,0.13)$ & $(0.32,0.20)$ & $(0.27,0.13)$ \\
\hline
\end{tabular}

Step 3: By combining the evaluations of all specialists/policy makers, construct the matrix $[\beta]_{6 \times(5+3)}$. (see Table 5)

Table 5. Group generalized q́-rung orthopair fuzzy assessment matrix $[\beta]_{6 \times 8}$.

\begin{tabular}{|c|c|c|c|c|}
\hline & $\iota_{1}$ & $\lambda_{2}$ & $\curlywedge_{3}$ & $\curlywedge_{4}$ \\
\hline$\widetilde{U}_{1}$ & $(0.67,0.21)$ & $(0.57,0.11)$ & $(0.86,0.14)$ & $(0.72,0.21)$ \\
\hline$\widetilde{U}_{2}$ & $(0.35,0.38)$ & $(0.21,0.68)$ & $(0.51,0.66)$ & $(0.27,0.38)$ \\
\hline$\widetilde{U}_{3}$ & $(0.41,0.17)$ & $(0.35,0.45)$ & $(0.67,0.51)$ & $(0.28,0.78)$ \\
\hline$\widetilde{U}_{4}$ & $(0.13,0.66)$ & $(0.32,0.31)$ & $(0.35,0.61)$ & $(0.31,0.52)$ \\
\hline$\widetilde{U}_{5}$ & $(0.67,0.21)$ & $(0.57,0.32)$ & $(0.86,0.14)$ & $(0.70,0.20)$ \\
\hline$\widetilde{U}_{6}$ & $(0.46,0.38)$ & $(0.32,0.68)$ & $(0.62,0.66)$ & $(0.38,0.28)$ \\
\hline & $\curlywedge_{5}$ & $\mathfrak{g}_{1}$ & $\mathfrak{g}_{2}$ & $\mathfrak{g}_{3}$ \\
\hline$\widetilde{\cup}_{1}$ & $(0.62,0.21)$ & $(0.72,0.25)$ & $(0.68,0.12)$ & $(0.58,0.22)$ \\
\hline$\widetilde{U}_{2}$ & $(0.72,0.23)$ & $(0.21,0.35)$ & $(0.35,0.63)$ & $(0.82,0.26)$ \\
\hline$\widetilde{U}_{3}$ & $(0.34,0.21)$ & $(0.45,0.26)$ & $(0.35,0.71)$ & $(0.67,0.52)$ \\
\hline$\widetilde{U}_{4}$ & $(0.81,0.24)$ & $(0.14,0.62)$ & $(0.25,0.14)$ & $(0.38,0.27)$ \\
\hline$\widetilde{U}_{5}$ & $(0.72,0.20)$ & $(0.20,0.17)$ & $(0.31,0.23)$ & $(0.26,0.25)$ \\
\hline$\widetilde{U}_{6}$ & $(0.81,0.31)$ & $(0.24,0.13)$ & $(0.32,0.20)$ & $(0.27,0.13)$ \\
\hline
\end{tabular}

Step 4: Calculate $\aleph_{\mathfrak{i}}$ for all q́q-ROFNs using GGQROWG-operator. The results obtained by GGQROFOWG-operator and GGQROFHG-operator are also mentioned in Table 6. For GGQROFHG-operator, policy makers/decision experts will determine a standard weight vector $(0.4,0.2,0.2,0.1,0.1)$ in accordance with evaluation attributes to hybridize the specified information. The hybridization is slightly different method to choose the suitable strategy and very useful to identify the precise conclusions. Ranking can be obtained by using one of three given operators.

Table 6. Aggregated matrix by using aggregation operators.

\begin{tabular}{cccc}
\hline & GGQROFWG & GGQROFOWG & GGQROFHG \\
\hline$\widetilde{U}_{1}$ & $(0.789337,0.040080)$ & $(0.766308,0.036567)$ & $(0.740615,0.079633)$ \\
$\widetilde{U}_{2}$ & $(0.581194,0.227714)$ & $(0.519988,0.271544)$ & $(0.382272,0.357161)$ \\
$\widetilde{U}_{3}$ & $(0.565361,0.310873)$ & $(0.551443,0.364875)$ & $(0.539303,0.239981)$ \\
$\widetilde{\Psi}_{4}$ & $(0.460982,0.194678)$ & $(0.328874,0.232439)$ & $(0.343440,0.246197)$ \\
$\widetilde{U}_{5}$ & $(0.383551,0.049236)$ & $(0.690241,0.054126)$ & $(0.629404,0.090020)$ \\
$\widetilde{U}_{6}$ & $(0.585816,0.077892)$ & $(0.463008,0.093438)$ & $(0.447749,0.097445)$ \\
\hline
\end{tabular}

Step 5: Calculate the score values for each $\aleph_{i}$, as given in Table 7.

Table 7. Score values of $\aleph_{i}$.

\begin{tabular}{cccc}
\hline & GGQROFWG & GGQROFOWG & GGQROFHG \\
\hline$\widetilde{\tau}\left(\aleph_{1}\right)$ & 0.491734 & 0.449948 & 0.405730 \\
$\widetilde{\neg}\left(\aleph_{2}\right)$ & 0.184511 & 0.119768 & 0.010301 \\
$\widetilde{\tau}\left(\aleph_{3}\right)$ & 0.150664 & 0.119110 & 0.143034 \\
$\widetilde{\tau}\left(\aleph_{4}\right)$ & 0.090582 & 0.023012 & 0.025586 \\
$\widetilde{\tau}\left(\aleph_{5}\right)$ & 0.056305 & 0.328694 & 0.248608 \\
$\widetilde{\tau}\left(\aleph_{6}\right)$ & 0.200567 & 0.098442 & 0.088839 \\
\hline
\end{tabular}


Step 6: The order of preferences of the alternatives by using GGQROPFWG-operaor, GGQROPFOWGoperator, and GGQROPFHG-operator are given in Table 8 and the graphical representations are given in Figures 6-8 respectively.

Table 8. Final ranking of alternatives.

\begin{tabular}{ll}
\hline \multicolumn{1}{c}{ Method } & Ranking of Alternatives \\
\hline GGQROFWG-operator & $\widetilde{U}_{1} \succ \widetilde{U}_{6} \succ \widetilde{U}_{2} \succ \widetilde{U}_{3} \succ \mathbb{U}_{4} \succ \mathbb{U}_{5}$ \\
GGQROFOWG-operator & $\widetilde{U}_{1} \succ \widetilde{U}_{5} \succ \widetilde{U}_{2} \succ \widetilde{U}_{3} \succ \widetilde{U}_{6} \succ \widetilde{U}_{4}$ \\
GGQROFHG-operator & $\widetilde{U}_{1} \succ \widetilde{U}_{5} \succ \widetilde{U}_{3} \succ \mathbb{U}_{6} \succ \widetilde{U}_{4} \succ \mathbb{U}_{2}$ \\
\hline
\end{tabular}

The final ranking shows that $\ddot{\alpha_{1}}$ is the best strategy to control the water loss. It should be emphasized that all aggregation operators provides nearby similar outcomes. By the reason of different techniques of provided aggregation operators, the little difference in the ranking of strategies can be observed but the optimal outcomes acquired from all proposed aggregation operators are precise and provide appropriate order of priority regarding the choice of suitable strategy. The highly preferred option is pressure management that is focused by policy makers/decision experts. The execution of this strategy comprises the formation of pressure zones to sustain the pressure range and the pressure would have to be restricted as required, by the use of pressure reducers. The main objective of this provided strategy is the inadequate explanation of pressure zones related with subsequent components: big difference in elevations which leads high level water pressure, leading finally to physical losses of water with breakage of pipes and irregular water supply, which is connected with highly esteemed pumping equipment, which leads to pressure relief and subsequently gives rise to the pipe bursts. The pressure management strategy is applied by many developed countries to control their water loss.

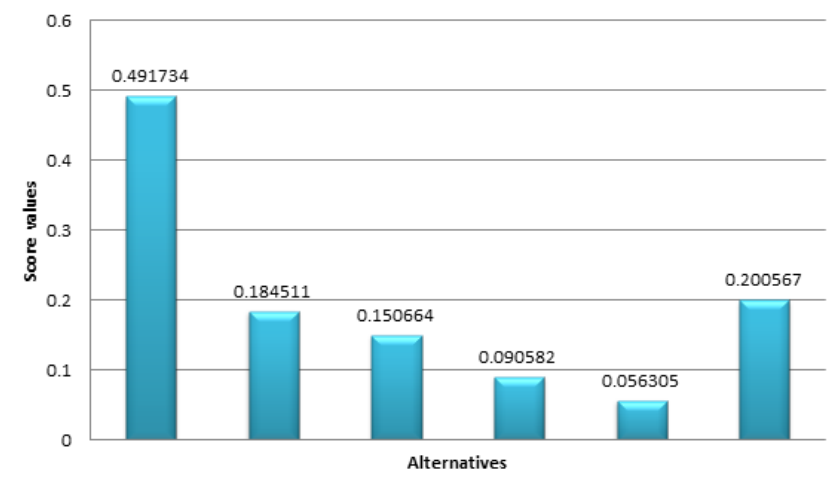

Figure 6. Score values obtained by GGQROFWG-operator.

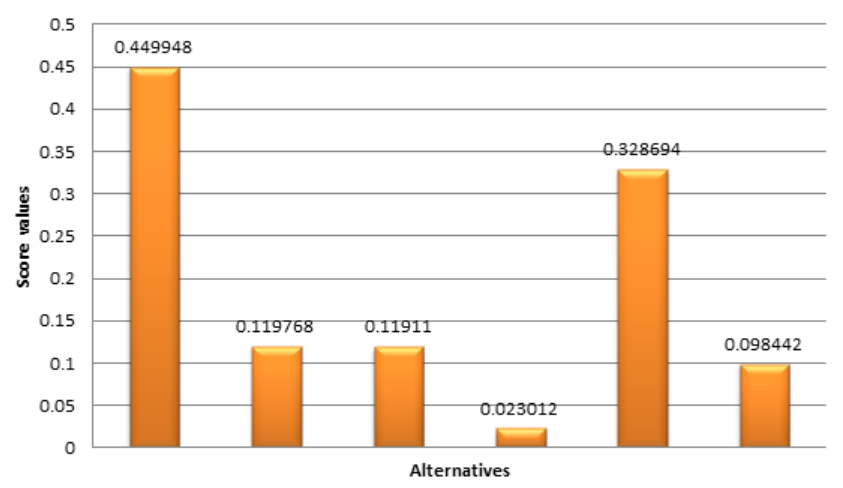

Figure 7. Score values obtained by GGQROFOWG-operator. 


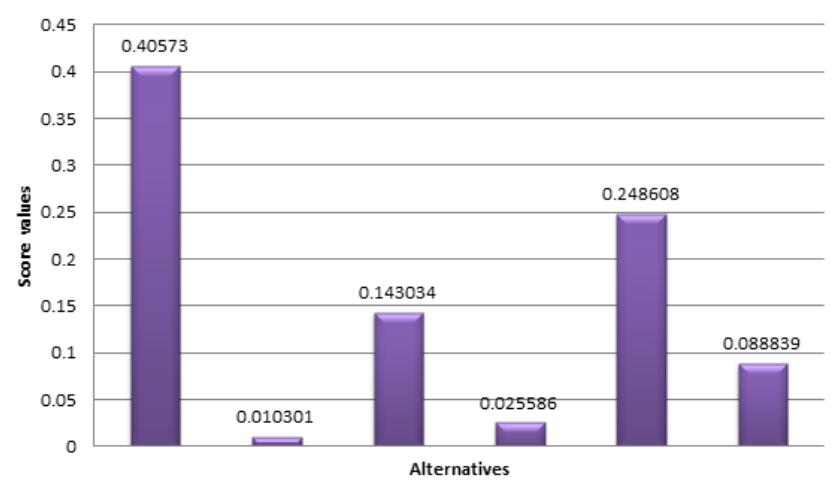

Figure 8. Score values obtained by GGQROFHG-operator.

\subsection{Sensitive Analysis}

In the presented section, we investigate the impact, by considering only one specialist/decision expert on decision analysis. If the analysis done on the recommendation of one specialist/decision expert regarding authenticity of the provided information, then we have the following conclusions:

(1) If only $\mathfrak{g}_{1}$ is to be considered, then by the above analysis we get the ranking $\widetilde{\mathbb{U}}_{1} \succ \widetilde{\mathbb{U}}_{5} \succ \widetilde{\mathbb{U}}_{6} \succ$ $\widetilde{U}_{3} \succ \widetilde{\mathbb{U}}_{2} \succ \widetilde{\cup}_{4}$. The score values are given in Figure 9 .

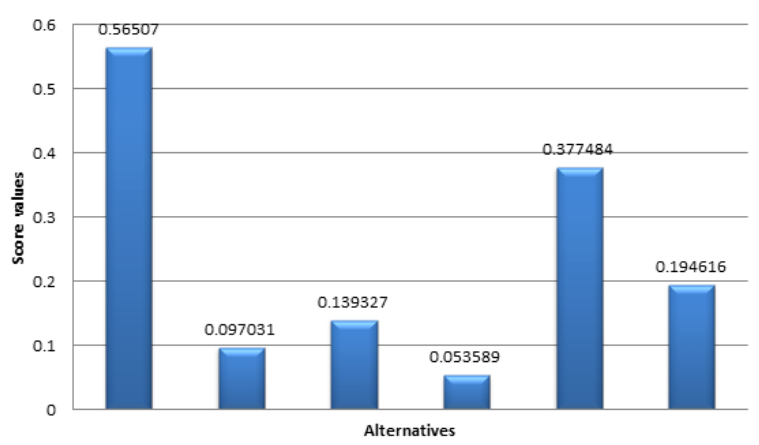

Figure 9. Score values when only $\check{\mathfrak{g}}_{1}$ is considered.

(2) If only $\mathfrak{g}_{2}$ is to be considered, then by the above analysis, we get the ranking $\widetilde{\cup}_{1} \succ \widetilde{U}_{5} \succ \widetilde{U}_{6} \succ$ $\widetilde{U}_{2} \succ \widetilde{\mathbb{U}}_{4} \succ \widetilde{\mathbb{U}}_{3}$. The score values are given in Figure 10.

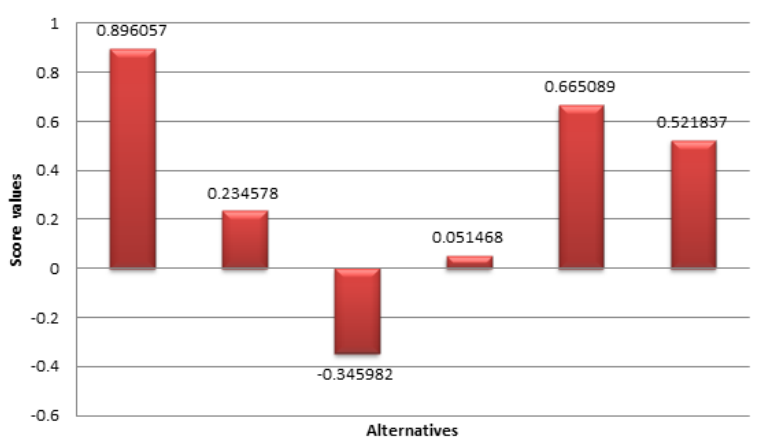

Figure 10. Score values when only $\check{\mathfrak{g}}_{2}$ is considered.

(3) If only $\mathfrak{g}_{3}$ is to be considered, then by the above analysis we get the ranking $\widetilde{U}_{1} \succ \widetilde{U}_{6} \succ \widetilde{U}_{5} \succ$ $\widetilde{U}_{4} \succ \widetilde{\mathbb{U}}_{3} \succ \widetilde{U}_{2}$. The score values are given in Figure 11 . 


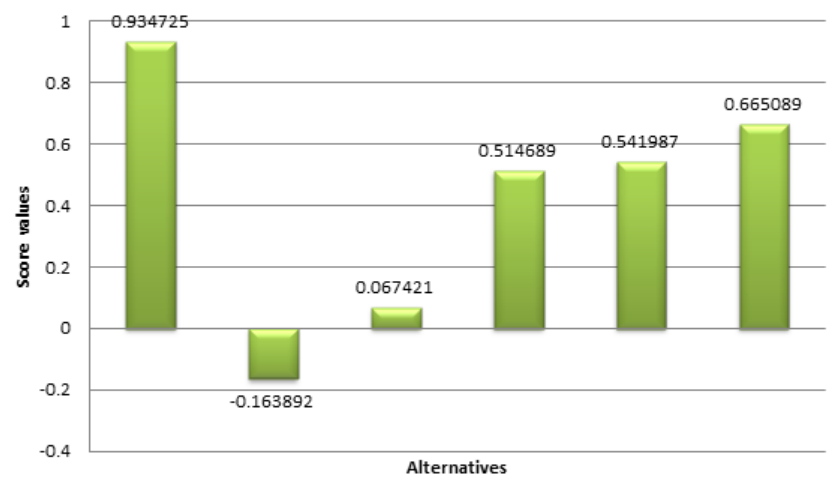

Figure 11. Score values when only $\check{\mathfrak{g}}_{3}$ is considered.

The final ranking achieved by taking into account only a single policy maker/decision expert, is changed but the suitable universal element remained same, which is signifying and demonstrating that each policy maker/specialist has own priorities and values of evaluation attributes, due to his/her own awareness, confessions, knowledge, and personal experiences.

\subsection{Comparison Analysis}

To demonstrate the productiveness and eminent benefits of the established aggregation operators, the same numerical example is solved by utilizing other aggregation operators including GPFEWG-operator, QROFWA-operator, QROFWG-operator, QROFEWG-operator, QROFEOWG-operator by ignoring the additional preference matrix in some existing operators. Different aggregation operators have distinct classification of strategies so they are able to sustain a little difference in accordance with their consultation. It can be noted in comparison, the suitable choice developed by any aggregation operator, is significant and acknowledges the viability and efficiency of the proposed aggregation operators. The comparison analysis of final rankings of all aggregation operators is given in Table 9.

Table 9. Comparison analysis of final ranking with existing aggregation operators.

\begin{tabular}{|c|c|c|}
\hline Method & Ranking of Alternatives & Optimal Alternative \\
\hline GGQROFWG operator (Proposed) & $\widetilde{U}_{1} \succ \widetilde{U}_{6} \succ \widetilde{U}_{2} \succ \widetilde{U}_{3} \succ \widetilde{U}_{4} \succ \widetilde{U}_{5}$ & $\widetilde{U}_{1}$ \\
\hline GGQROFOWG operator (Proposed) & ${\underset{\sim}{\sim}}_{1} \succ \widetilde{\sim}_{5} \succ \widetilde{\sim}_{2} \succ \widetilde{\sim}_{3} \succ \widetilde{\sim}_{6} \succ \widetilde{\sim}_{4}$ & ${\underset{\sim}{\Psi}}_{1}$ \\
\hline GGQROFHG operator (Proposed) & $\widetilde{U}_{1} \succ \widetilde{U}_{5} \succ \widetilde{U}_{3} \succ \widetilde{U}_{6} \succ \widetilde{U}_{4} \succ \widetilde{\mathbb{U}}_{2}$ & $\widetilde{\mathbb{U}}_{1}$ \\
\hline q́-ROFEPWA operator (Riaz et al. [51]) & $\widetilde{U}_{1} \succ \widetilde{\mathbb{U}}_{3} \succ \widetilde{U}_{5} \succ \widetilde{\mathbb{U}}_{6} \succ \widetilde{U}_{4} \succ \widetilde{U}_{2}$ & $\widetilde{\cup}_{1}$ \\
\hline q́-ROFEPWG operator (Riaz et al. [51]) & $\widetilde{U}_{1} \succ \widetilde{\mathbb{U}}_{3} \succ \widetilde{U}_{5} \succ \widetilde{\mathbb{U}}_{6} \succ \widetilde{U}_{4} \succ \widetilde{\mathbb{U}}_{2}$ & $\widetilde{\mathbb{U}}_{1}$ \\
\hline q́-ROFWG operator (Liu and Wang [45]) & ${\underset{\sim}{\sim}}_{1} \succ{\underset{\sim}{\mathbb{U}}}_{2} \succ \widetilde{\sim}_{3} \succ \widetilde{\sim}_{6} \succ \widetilde{\sim}_{4} \succ \widetilde{\sim}_{5}$ & $\mathbb{\sim}_{1}$ \\
\hline q́-ROFOWG operator (Liu and Wang [45]) & $\widetilde{\mathbb{U}}_{1} \succ \widetilde{\mathbb{U}}_{2} \succ \widetilde{\mathbb{U}}_{6} \succ \widetilde{\mathbb{U}}_{3} \succ \widetilde{\mathbb{U}}_{4} \succ \widetilde{\mathbb{U}}_{5}$ & $\widetilde{\mathbb{U}}_{1}$ \\
\hline q́-ROFWA operator (Liu and Wang [45]) & 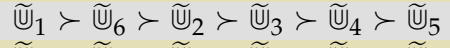 & $\widetilde{\sim}_{1}$ \\
\hline q́-ROFOWA operator (Liu and Wang [45]) & $\widetilde{\sim}_{1} \succ \widetilde{\sim}_{6} \succ \widetilde{\mathbb{U}}_{2} \succ \widetilde{\sim}_{3} \succ \widetilde{\mathbb{U}}_{4} \succ \widetilde{\sim}_{5}$ & $\widetilde{\cup}_{1}$ \\
\hline q́-ROFEWG operator(Riaz et al. [48]) & $\widetilde{U}_{1} \succ \widetilde{U}_{5} \succ \widetilde{U}_{3} \succ \widetilde{U}_{4} \succ \widetilde{U}_{2} \succ \widetilde{U}_{6}$ & $\widetilde{U}_{1}$ \\
\hline q́-ROFEOWG operator(Riaz et al. [48]) & ${\underset{\sim}{\sim}}_{1} \succ \widetilde{\sim}_{3} \succ \widetilde{\mathbb{U}}_{5} \succ \widetilde{\sim}_{6} \succ \widetilde{\sim}_{4} \succ \widetilde{\sim}_{2}^{\widetilde{U}_{2}}$ & $\mathbb{\sim}_{1}$ \\
\hline q́-ROFPWA operator (Riaz et al. [49]) & $\widetilde{\mathbb{U}}_{1} \succ \widetilde{\mathbb{U}}_{3} \succ \widetilde{U}_{5} \succ \widetilde{\mathbb{U}}_{6} \succ \widetilde{U}_{4} \succ \widetilde{\mathbb{U}}_{2}$ & $\widetilde{\mathbb{U}}_{1}$ \\
\hline q́-ROFPWG operator (Riaz et al. [49]) & $\widetilde{U}_{1} \succ \widetilde{U}_{3} \succ \widetilde{U}_{5} \succ \widetilde{U}_{6} \succ \widetilde{U}_{4} \succ \widetilde{U}_{2}$ & $\widetilde{U}_{1}$ \\
\hline q́-ROFHWAGA operator (Riaz et al. [50]) & $\widetilde{U}_{1} \succ \widetilde{U}_{3} \succ \widetilde{U}_{5} \succ \widetilde{U}_{6} \succ \widetilde{U}_{4} \succ \widetilde{\mathbb{U}}_{2}$ & $\widetilde{U}_{1}$ \\
\hline q́-ROFHOWAGA operator (Riaz et al. [50]) & $\widetilde{\mathbb{U}}_{1} \succ \widetilde{\mathbb{U}}_{3} \succ \widetilde{U}_{5} \succ \widetilde{\mathbb{U}}_{6} \succ \widetilde{\mathbb{U}}_{2} \succ \widetilde{\mathbb{U}}_{4}$ & $\widetilde{U}_{1}$ \\
\hline
\end{tabular}

Consequently the provided method establish the similar alternative as achieved by different aggregation operators which states that the provided method is beneficial and conceivable.

\section{Conclusions}

A variety of methods have been suggested to incorporate $\mathfrak{q}-R O F$ values. Even though prevailing $\mathfrak{q}$-ROF aggregation operators were established under the presumption that decision experts have a profound knowledge, these kinds of circumstances were not met while handling the realistic issues, 
as the policy maker/decision experts priorities regarding alternatives are characteristic of one's own apprehension. Consequently it is required to establish a few different and modern approaches. To deal with this problem, the idea of GQROFS is established by integrating the concept of GP of the other specialist/experts and provides the structure for evaluating the morality of the provided data in initial $\mathfrak{q}$-ROFS to eliminate any distortion in the preferences of senior expert. The most important advantage of addition of generalized parameter is to overcome the chances of mistakes resulting from inaccurate information. This theory is extended to group generalized parameter by integrating the evaluation of different specialists/decision makers which will decrease the influence of single decision expert's choices and will approximate the far more realistic condition under q́-ROF environment. In this paper, we developed q́ -ROF geometric aggregation operator under generalized parameter and $\mathfrak{q}$-ROF geometric aggregation operator under group-based generalized parameter. The viability and effectiveness of the proposed aggregation operators are demonstrated by a numerical example. This examination is favorable to utilities of water in respect of achievement a clear idea and evaluation of elements of water loss management strategies, their collaborations and proportions which are not restricted to economic zone, but are expanded to cover environmental, potentially health, and security concerns. The outcomes deliberate the policy maker's concerns in considering the most efficient strategies to reduce the shortages in the water supply system connected with the adoption of unsystematic supply scheme. For further studies, taking into account the advanced simulation capabilities of q-ROFSs, in the q-ROF context we may further examine different kinds of AOs and apply them to realistic decision-making situations. Moreover, the methodological advances for many fields like machine learning, robotics, green supply chain management (GSCM), medical diagnosis, weather forecasting, intelligence, informatics, and sustainable energy planning decision making are promising areas for future studies.

Author Contributions: M.R., A.R., H.K., D.P., and Y.-M.C. conceived of and worked together to achieve this manuscript; M.R., H.M.A.F., D.P., and Y.-M.C. constructed the ideas and algorithms for data analysis and designed the model of the manuscript; A.R., H.K., H.M.A.F., and M.R. processed the data collection and wrote the paper. All authors read and agreed to the published version of the manuscript.

Funding: This research received no external funding.

Acknowledgments: The authors are highly thankful to the Editor-in-Chief and referees for their valuable comments and suggestions for the improvement of our manuscript.

Conflicts of Interest: The authors declare that they have no conflict of interest.

\section{References}

1. Kingdom, B.; Liemberger, R.; Marin, P. The Challenge of Reducing Non-revenue Water (NRW) in Developing Countries; The World Bank: Washington, DC, USA, 2006.

2. Malmqvist, P.A.; Heinicke, G.; Karrman, E.; Stenstrom, T.A.; Svensson, G. Urban water in context. In Strategic Planning of Sustainable Urban Water Management; Malmqvist, P.A., Heinecke, G., Karrman, E., Stenstrom, T.A., Eds.; IWA Publishing: London, UK, 2006.

3. Mugabi, J.; Kayaga, S.; Njiru, C. Strategic planning for water utilities in developing countries. Util. Policy 2007, 15, 1-8. [CrossRef]

4. Loureiro, D.; Amado, C.; Martins, A.; Vitorino, D.; Mamade, A.; Coelho, S.T. Water distribution systems flow monitoring and anomalous event detection: A practical approach. Urban Water J. 2016, 13, 1-11. [CrossRef]

5. Mutikanga, H.E.; Sharma, S.K.; Vairavamoorthy, K. Multicriteria decision analysis: A strategic planning tool for water loss management. Water Resour. Manag. 2011, 25, 3947-3969. [CrossRef]

6. Morais, D.C.; Almeida, A.T. Group decision making for leakage management strategy of water network. Resour. Conserv. Recycl. 2007, 52, 441-458. [CrossRef]

7. Silva, V.B.S.; Morais, D.C.; Almeida, A.T. A multicriteria group decision model to support watershed committees in Brazil. Water Resour. Manag. 2010, 24, 4075-4091. [CrossRef]

8. Bouchard, C.; Abi-zeid, I.; Beauchamp, N.; Lamontagne, L.; Desrosiers, J. Multicriteria decision analysis for the selection of a small drinking water system. Water Suppl. Res. Technol. AQUA 2010, 59, 230-242. [CrossRef] 
9. Afshar, A.; Marino, M.A.; Afshar, S.M. Fuzzy TOPSIS multicriteria decision analysis applied to Karun reservoir system. Water Resour. Manag. 2011, 25, 545-563. [CrossRef]

10. Shaher, H.Z.; Shaheen, H.; Samhan, S.; Rabi, A.; Al-Wadi, F.; Fuchs-Hanusch, D. Utilizing analytic hierarchy process (AHP) for decision making in water loss management of intermittent water supply systems. J. Water Sanit. Hyg. Dev. 2016, 6, 534-546.

11. Zadeh, L.A. Fuzzy sets. Inf. Control. 1965, 8, 338-353. [CrossRef]

12. Atanassov, K.T. Intuitionistic fuzzy sets. Fuzzy Sets Syst. 1986, 20, 87-96. [CrossRef]

13. Yager, R.R. Pythagorean fuzzy subsets. In Prcoceedings of the 2013 Joint IFSA World Congress and NAFIPS Annual Meeting (IFSA/NAFIPS), Edmonton, AB, Canada, 24-28 June 2013; pp. 57-61.

14. Yager, R.R.; Abbasov, A.M. Pythagorean membership grades, complex numbers, and decision making. Int. J. Intell. Syst. 2013, 28, 436-452. [CrossRef]

15. Yager, R.R. Pythagorean membership grades in multi-criteria decision making. IEEE Trans. Fuzzy Syst. 2014, 22, 958-965. [CrossRef]

16. Ali, M.I. A note on soft sets, rough soft sets and fuzzy soft sets. Appl. Soft Comput. 2011, 11, 3329-3332.

17. Wang, Y.; Li, Z.; Tang, Z.; Zeng, G. A GIS-based spatial multi-criteria approach for flood risk management in the Dongting Lake Region, Hunan, Central China. Water Resour. Manag. 2011, 25, 3465-3484. [CrossRef]

18. Wang, H.; Smarandache, F.; Zhang, Y.Q.; Sunderraman, R. Single valued neutrosophic sets. Multispace Multistruct. 2010, 4, 410-413.

19. Kaur, G.; Garg, H. Cubic intuitionistic fuzzy aggregation operators. Int. J. Uncertain. Quantif. 2018, 8, 405-427. [CrossRef]

20. Kumar, K.; Garg, H. TOPSIS method based on the connection number of set pair analysis under intervalvalued intuitionistic fuzzy set environment. Comput. Appl. Math. 2018, 37, 1319-1329. [CrossRef]

21. Peng, X.D.; Yang, Y. Some results for Pythagorean fuzzy sets. Int. J. Intell. Syst. 2015, 30, 1133-1160. [CrossRef]

22. Peng, X.D.; Yang, Y.Y.; Song, J.; Jiang, Y. Pythagorean Fuzzy Soft Set and Its Application. Comput. Eng. 2015, 41, 224-229.

23. Riaz, M.; Hashmi, M.R. Linear Diophantine fuzzy set and its applications towards multi-attribute decision making problems. J. Intell. Fuzzy Syst. 2019, 37, 5417-5439. [CrossRef]

24. Riaz, M.; Tehrim, S.T. Cubic bipolar fuzzy set with application to multi-criteria group decision making using geometric aggregation operators. Soft Comput. 2020. [CrossRef]

25. Riaz, M.; Tehrim, S.T. A Robust Extension of VIKOR Method for Bipolar Fuzzy Sets using Connection Numbers of SPA Theory Based Metric Spaces. Artif. Intell. Rev. 2020. [CrossRef]

26. Sharma, H.K.; Kumari, K.; Kar, S. A rough set approach for forecasting models. Decis. Mak. Appl. Manag. Eng. 2020, 3, 1-21. [CrossRef]

27. Petrovic, I.; Kankaras, M. A hybridized IT2FS-DEMATEL-AHP-TOPSIS multicriteria decision making approach: Case study of selection and evaluation of criteria for determination of air traffic control radar position. Decis. Mak. Appl. Manag. Eng. 2020, 3, 146-164. [CrossRef]

28. Yager, R.R. Generalized Orthopair Fuzzy sets. IEEE Trans. Fuzzy Syst. 2017, 25, 1220-1230. [CrossRef]

29. Peng, X.D.; Yuan, H.Y.; Yang, Y. Pythagorean fuzzy information measures and their applications. Int. J. Intell. Syst. 2017, 32, 991-1029. [CrossRef]

30. Ali, M.I. Another view on q́-Rung orthopair fuzzy sets. Int. J. Intell. Syst. 2018, 33, 2139-2153. [CrossRef]

31. Chen, J.; Li, S.; Ma, S.; Wang, X. m-Polar Fuzzy Sets: An Extension of Bipolar Fuzzy Sets. Sci. World J. 2014. [CrossRef]

32. Chi, P.P.; Lui, P.D. An extended TOPSIS method for the multiple ttribute decision making problems based on interval neutrosophic set. Neutrosophic Sets Syst. 2013, 1, 63-70.

33. Feng, F.; Jun, Y.B.; Liu, X.; Li, L. An adjustable approach to fuzzy soft set based decision making. J. Comput. Appl. Math. 2010, 234, 10-20. [CrossRef]

34. Feng, F.; Li, C.; Davvaz, B.; Ali, M.I. Soft sets combined with fuzzy sets and rough sets; A tentative approach. Soft Comput. 2010, 14, 899-911. [CrossRef]

35. Feng, F.; Liu, X.Y.; Leoreanu-Fotea, V.; Jun, Y.B. Soft sets and soft rough sets. Inf. Sci. 2011, 181, $1125-1137$. [CrossRef] 
36. Feng, F.; Fujita, H.; Ali, M.I.; Yager, R.R.; Liu, X. Another view on generalized intuitionistic fuzzy soft sets and related multi-attribute decision making methods. IEEE Trans. Fuzzy Syst. 2019, 27, 474-488. [CrossRef]

37. Garg, H. Generalized pythagorean fuzzy geometric aggregation operator uing Einstein t-norm and t-conorm for Multi-criteria decision making process. Int. J. Intell. Syst. 2017, 32, 597-630. [CrossRef]

38. Garg, H.; Arora, R. Generalized intuitionistic fuzzy soft power aggregation operator based on t-norm and their application in multicriteria decision-making. Int. J. Intell. Syst. 2019, 34, 215-246. [CrossRef]

39. Garg, H.; Arora, R. Dual hesitant fuzzy soft aggregation operators and their applicatio in decision-making. Cogn. Comput. 2018, 10, 769-789. [CrossRef]

40. Garg, H.; Arora, R. A nonlinear-programming methodology for multi-attribute decision-making problem with interval-valued intuitionistic fuzzy soft sets information. Appl. Intell. 2018, 48, 2031-2046. [CrossRef]

41. Garg, H.; Arora, R. Novel scaled prioritized intuitionistic fuzzy soft interaction averaging aggregation operators and their application to multi criteria decision-making. Eng. Appl. Artif. Intell. EAAI 2018, 71, 100-112. [CrossRef]

42. Jose, S.; Kuriaskose, S. Aggregation operators, score function and accuracy function for multi criteria decision making in intuitionistic fuzzy context. Notes Intuit. Fuzzy Sets 2014, 20, 40-44.

43. Joshi, B.P. Pythagorean fuzzy average aggregation operators based on generalized and group-generalized parameter with application in MCDM problems. Int. J. Intell. Syst. 2019, 34, 895-919. [CrossRef]

44. Karaaslan, F. Neutrosophic Soft Set with Applications in Decision Making. Int. J. Inf. Sci. Intell. Syst. 2015, 4, 1-20.

45. Liu, P.; Wang, P. Some q-rung orthopair fuzzy aggregation operator and their application to multi-attribute decision making. Int. J. Intell. Syst. 2018, 33, 2259-2280.

46. Liu, Y.; Zhang, H.; Wu, Y.; Dong, Y. Ranking range based approach to MADM under incomplete context and its application in venture investment evaluation. Technol. Econ. Dev. Econ. 2019, in press. [CrossRef]

47. Peng, X.D.; Dai, J. Approaches to single-valued neutrosophic MADM based on MABAC, TOPSIS and new similarity measure with score function. Neural Comput. Appl. 2018, 29, 939-954. [CrossRef]

48. Riaz, M.; Salabun, W.; Farid, H.M.A.; Ali, N.; Watróbski, J. A robust q-rung orthopair fuzzy information aggregation using Einstein operations with application to sustainable energy planning decision management. Energies 2020, 13, 2155. [CrossRef]

49. Riaz, M.; Pamucar, D.; Farid, H.M.A.; Hashmi, M.R. q-Rung Orthopair Fuzzy Prioritized Aggregation Operators and Their Application Towards Green Supplier Chain Management. Symmetry 2020, 12, 976. [CrossRef]

50. Riaz, M.; Karaaslan, F.; Farid, H.M.A.; Hashmi, M.R. Some q-rung orthopair fuzzy hybrid aggregation operators and TOPSIS method for multi-attribute decision-making. J. Intell. Fuzzy Syst. 2020. JIFS-192114. [CrossRef]

51. Riaz, M.; Farid, H.M.A.; Kalsoom, H.; Pamucar, D.; Chu, Y.M. A Robust q-rung orthopair fuzzy Einstein prioritized aggregation operators with application towards MCGDM. Symmetry 2020, 12, 1058. [CrossRef]

52. Xu, Z.S. Intuitionistic fuzzy aggregation operators. IEEE Trans. Fuzzy Syst. 2007, 15, 1179-1187.

53. Xu, Z.S.; Cai, X.Q. Intuitionistic Fuzzy Information Aggregation: Theory and Applications; Science Press: Beijing, China; Springer: Berlin/Heidelberg, Germany, 2012.

54. Xu, Z.S. Studies in Fuzziness and Soft Computing: Hesitant Fuzzy Sets Theory; Springer International Publishing: Cham, Switzerland, 2014.

55. Yager, R.R. On ordered weighted averaging aggregation operators in multi-criteria decision making. IEEE Trans. Syst. Man Cybern. 1988, 18, 183-190. [CrossRef]

56. Ye, J. Interval-valued hesitant fuzzy prioritized weighted aggregation operators for multi attribute decisionmaking. J. Algorithms Comput. Technol. 2013, 8, 179-192. [CrossRef]

57. Ye, J. Linguistic neutrosophic cubic numbers and their multiple attribute decision-making method. Information 2017, 8, 1-11. [CrossRef]

58. Zhan, J.; Liu, Q.; Davvaz, B. A new rough set theory: Rough soft hemirings. J. Intell. Fuzzy Syst. 2015, 28, 1687-1697. [CrossRef]

59. Zhan, J.; Alcantud, J.C.R. A novel type of soft rough covering and its application to multi-criteria group decision-making. Artif. Intell. Rev. 2018. [CrossRef] 
60. Zhang, L.; Zhan, J. Fuzzy soft $\beta$-covering based fuzzy rough sets and corresponding decision-making applications. Int. J. Mach. Learn. Cybern. 2018, 10, 1487-1502. [CrossRef]

61. Zhang, L.; Zhan, J. Novel classes of fuzzy soft $\beta$-coverings-based fuzzy rough sets with applications to multi-criteria fuzzy group decision-making. Soft Comput. 2018, 23, 5327-5351. [CrossRef]

62. Zhang, L.; Zhan, J.; Xu, Z.S. Covering-based generalized IF rough sets with applications to multi-attribute decision-making. Inf. Sci. 2019, 478, 275-302. [CrossRef]

(C) 2020 by the authors. Licensee MDPI, Basel, Switzerland. This article is an open access article distributed under the terms and conditions of the Creative Commons Attribution (CC BY) license (http://creativecommons.org/licenses/by/4.0/). 